\title{
The miniJPAS survey: A preview of the Universe in 56 colors ${ }^{\star}$
}

S. Bonoli ${ }^{1,2,3}$, A. Marín-Franch ${ }^{4}$, J. Varela ${ }^{4}$, H. Vázquez Ramió ${ }^{4}$, L. R. Abramo ${ }^{5}$, A. J. Cenarro ${ }^{4}$, R. A. Dupke $^{6,30,31}$, J. M. Vílchez ${ }^{7}$, D. Cristóbal-Hornillos ${ }^{1}$, R. M. González Delgado ${ }^{7}$, C. Hernández-Monteagudo ${ }^{4}$, C. López-Sanjuan ${ }^{4}$, D. J. Muniesa ${ }^{1}$, T. Civera ${ }^{1}$, A. Ederoclite ${ }^{13}$, A. Hernán-Caballero ${ }^{1}$, V. Marra ${ }^{8,25,26}$, P. O. Baqui ${ }^{8}$, A. Cortesi ${ }^{19}$,

E. S. Cypriano ${ }^{13}$, S. Daflon ${ }^{6}$, A. L. de Amorim ${ }^{23}$, L. A. Díaz-García ${ }^{11}$, J. M. Diego ${ }^{12}$, G. Martínez-Solaeche ${ }^{7}$, E. Pérez ${ }^{7}$,

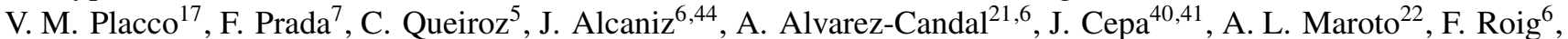
B. B. Siffert ${ }^{15}$, K. Taylor $^{33}$, N. Benitez ${ }^{7}$, M. Moles ${ }^{1,7}$, L. Sodré Jr. ${ }^{13}$, S. Carneiro ${ }^{10}$, C. Mendes de Oliveira ${ }^{13}$, E. Abdalla ${ }^{5}$, R. E. Angulo ${ }^{2,3}$, M. Aparicio Resco ${ }^{22}$, A. Balaguera-Antolínez ${ }^{40,41}$, F. J. Ballesteros ${ }^{49}$, D. Brito-Silva ${ }^{13}$, T. Broadhurst ${ }^{2,3,39}$, E. R. Carrasco ${ }^{47}$, T. Castro ${ }^{24,25,26,27}$, R. Cid Fernandes ${ }^{23}$, P. Coelho ${ }^{13}$, R. B. de Melo ${ }^{6,31}$, L. Doubrawa ${ }^{13}$, A. Fernandez-Soto ${ }^{12,38}$, F. Ferrari ${ }^{14}$, A. Finoguenov ${ }^{36}$, R. García-Benito ${ }^{7}$, J. Iglesias-Páramo ${ }^{7}$, Y. Jiménez-Teja ${ }^{7}$, F. S. Kitaura ${ }^{40,41}$, J. Laur $^{28}$, P. A. A. Lopes ${ }^{19}$, G. Lucatelli ${ }^{14}$, V. J. Martínez ${ }^{38,49,50}$, M. Maturi ${ }^{34,35}$, R. A. Overzier ${ }^{6}$, C. Pigozzo ${ }^{10}$, M. Quartin ${ }^{18,19}$, J. E. Rodríguez-Martín , V. Salzano ${ }^{57}$, A. Tamm ${ }^{28}$, E. Tempel ${ }^{28}$, K. Umetsu ${ }^{11}$,

L. Valdivielso ${ }^{1}$, R. von Marttens ${ }^{6}$, A. Zitrin ${ }^{16}$, M. C. Díaz-Martín ${ }^{1}$, G. López-Alegre ${ }^{1}$, A. López-Sainz ${ }^{1}$, A. Yanes-Díaz ${ }^{1}$,

F. Rueda-Teruel ${ }^{1}$, S. Rueda-Teruel ${ }^{1}$, J. Abril Ibañez ${ }^{1,29}$, J. L Antón Bravo ${ }^{1}$, R. Bello Ferrer ${ }^{1}$, S. Bielsa ${ }^{1}$, J. M. Casino $^{1}$,

J. Castillo ${ }^{1}$, S. Chueca ${ }^{1}$, L. Cuesta ${ }^{1}$, J. Garzarán Calderaro $^{1}$, R. Iglesias-Marzoa ${ }^{1}$, C. Íniguez ${ }^{1}$, J. L. Lamadrid Gutierrez ${ }^{1}$,

F. Lopez-Martinez ${ }^{1}$, D. Lozano-Pérez ${ }^{1}$, N. Maícas Sacristán ${ }^{1}$, E. L. Molina-Ibáñez ${ }^{1}$, A. Moreno-Signes ${ }^{1}$,

S. Rodríguez Llano ${ }^{1}$, M. Royo Navarro ${ }^{1}$, V. Tilve Rua ${ }^{1}$, U. Andrade ${ }^{6}$, E. J. Alfaro ${ }^{7}$, S. Akras ${ }^{14}$, P. Arnalte-Mur ${ }^{49,50}$, B. Ascaso $^{54}$, C. E. Barbosa ${ }^{13}$, J. Beltrán Jiménez ${ }^{62}$, M. Benetti ${ }^{58,59}$, C. A. P. Bengaly ${ }^{6}$, A. Bernui ${ }^{6}$, J. J. Blanco-Pillado 3,39 , M. Borges Fernandes ${ }^{6}$, J. N. Bregman ${ }^{30}$, G. Bruzual ${ }^{52}$, G. Calderone ${ }^{25}$, J. M. Carvano ${ }^{6}$, L. Casarini ${ }^{9}$, J. Chaves-Montero ${ }^{2}$, A. L. Chies-Santos ${ }^{44}$, G. Coutinho de Carvalho ${ }^{48}$, P. Dimauro 6 , S. Duarte Puertas ${ }^{7}$, D. Figueruelo ${ }^{62}$,

J. I. González-Serrano ${ }^{12}$, M. A. Guerrero ${ }^{7}$, S. Gurung-López ${ }^{1,46}$, D. Herranz ${ }^{12}$, M. Huertas-Company ${ }^{40,41,42,43}$, J. A. Irwin ${ }^{31}$, D. Izquierdo-Villalba ${ }^{1}$, A. Kanaan ${ }^{23}$, C. Kehrig 7 , C. C. Kirkpatrick ${ }^{36}$, J. Lim ${ }^{55}$, A. R. Lopes ${ }^{6}$,

R. Lopes de Oliveira ${ }^{9,6}$, A. Marcos-Caballero ${ }^{39}$, D. Martínez-Delgado ${ }^{7}$, E. Martínez-González ${ }^{12}$,

G. Martínez-Somonte ${ }^{12,61}$, N. Oliveira ${ }^{6}$, A. A. Orsi ${ }^{1}$, M. Penna-Lima ${ }^{32}$, R. R. R. Reis ${ }^{18,19}$, D. Spinoso ${ }^{1}$, S. Tsujikawa $^{60}$,

P. Vielva ${ }^{12}$, A. Z. Vitorelli ${ }^{13}$, J. Q. Xia ${ }^{20}$, H. B. Yuan ${ }^{20}$, A. Arroyo-Polonio ${ }^{7}$, M. L. L. Dantas ${ }^{13}$, C. A. Galarza ${ }^{6}$,

D. R. Gonçalves ${ }^{19}$, R. S. Gonçalves ${ }^{6}$, J. E. Gonzalez ${ }^{6,44}$, A. H. Gonzalez ${ }^{53}$, N. Greisel ${ }^{1}$, F. Jiménez-Esteban ${ }^{63}$,

R. G. Landim ${ }^{37}$, D. Lazzaro ${ }^{6}$, G. Magris ${ }^{51}$, R. Monteiro-Oliveira ${ }^{13}$, C.B. Pereira ${ }^{6}$, M. J. Rebouças ${ }^{56}$, J. M. Rodriguez-Espinosa ${ }^{41}$, S. Santos da $\operatorname{Costa}^{6}$, and E. Telles ${ }^{6,45}$

(Affiliations can be found after the references)

Received 3 July 2020 / Accepted 14 March 2021

\begin{abstract}
The Javalambre-Physics of the Accelerating Universe Astrophysical Survey (J-PAS) will scan thousands of square degrees of the northern sky with a unique set of 56 filters using the dedicated $2.55 \mathrm{~m}$ Javalambre Survey Telescope (JST) at the Javalambre Astrophysical Observatory. Prior to the installation of the main camera $\left(4.2 \mathrm{deg}^{2}\right.$ field-of-view with 1.2 Gpixels), the JST was equipped with the JPAS-Pathfinder, a one CCD camera with a $0.3 \mathrm{deg}^{2}$ field-of-view and plate scale of 0.23 arcsec pixel $^{-1}$. To demonstrate the scientific potential of J-PAS, the JPAS-Pathfinder camera was used to perform miniJPAS, a $\sim 1 \mathrm{deg}^{2}$ survey of the AEGIS field (along the Extended Groth Strip). The field was observed with the 56 J-PAS filters, which include 54 narrow band $(F W H M \sim 145 \AA$ ) and two broader filters extending to the UV and the near-infrared, complemented by the $u, g, r, i$ SDSS broad band filters. In this miniJPAS survey overview paper, we present the miniJPAS data set (images and catalogs), as we highlight key aspects and applications of these unique spectro-photometric data and describe how to access the public data products. The data parameters reach depths of $\operatorname{mag}_{\mathrm{AB}} \simeq 22-23.5$ in the 54 narrow band filters and up to 24 in the broader filters ( $5 \sigma$ in a $3^{\prime \prime}$ aperture). The miniJPAS primary catalog contains more than 64000 sources detected in the $r$ band and with matched photometry in all other bands. This catalog is $99 \%$ complete at $r=23.6(r=22.7)$ mag for point-like (extended) sources. We show that our photometric redshifts have an accuracy better than $1 \%$ for all sources up to $r=22.5$, and a precision of $\leq 0.3 \%$ for a subset consisting of about half of the sample. On this basis, we outline several scientific applications of our data, including the study of spatially-resolved stellar populations of nearby galaxies, the analysis of the large scale structure up to $z \sim 0.9$, and the detection of large numbers of clusters and groups. Sub-percent redshift precision can also be reached for quasars, allowing for the study of the large-scale structure to be pushed to $z>2$. The miniJPAS survey demonstrates the capability of the J-PAS filter system to accurately characterize a broad variety of sources and paves the way for the upcoming arrival of J-PAS, which will multiply this data by three orders of magnitude.
\end{abstract}

Key words. surveys - techniques: photometric - astronomical databases: miscellaneous - stars: general - galaxies: general cosmology: observations

* miniJPAS data and associated value added catalogs are publicly available http://archive.cefca.es/catalogues/minijpaspdr201912 


\section{Introduction}

From the pioneering Sloan Digital Sky Survey (SDSS, York et al. 2000) to the most recent Dark Energy Survey (DES, Wester \& Dark Energy Survey Collaboration 2005), Panoramic Survey Telescope and Rapid Response System 1 (Pan-STARRS1, Chambers et al. 2016), and Hyper SuprimeCam Subaru Strategic Program (HSC-SSP, Aihara et al. 2018), large-area photometric surveys have been providing the community with a wealth of data for studies of all kinds of objects in our Universe. From stellar population studies to analyses of the large-scale structure, from the discovery of dwarf galaxies to gravitational lensing, every field in astronomy has benefited from these surveys. A leap forward is expected with the arrival of the Legacy Survey of Space and Time (LSST, Ivezić et al. 2019), which will provide deep broad-band imaging in the time domain. However, extragalactic photometric studies, in particular, often still prove challenging due to the fact that relatively few (broad band) filters have to be used to infer both the photometric redshifts and the physical properties of the sources, making expensive spectroscopic follow-up campaigns almost essential for the full exploitation of these imaging surveys. COMBO-17 (Wolf et al. 2003) was the first experiment to use a significantly larger number of bands, narrower in full-width-half-maximum (FWHM), allowing for a more precise characterization of the sources using photometric data alone (see also the seminal work of Hickson et al. 1994). The approach was then followed by other projects, such as COSMOS (Scoville et al. 2007), ALHAMBRA (Moles et al. 2008) and SHARDS (Pérez-González et al. 2013). All these experiments focused on the observation of small, but deep fields and opened the way for more extensive "spectro-photometric" observations. The PAU survey (e.g., Eriksen et al. 2019) is currently targeting tens of $\mathrm{deg}^{2}$ over a few known fields (e.g., CFHTLS, KiDS ) with 40 narrow band filters on the PAU Camera (Padilla et al. 2019), a guest instrument at the $4.2 \mathrm{~m}$ William Herschel Telescope. The J-PLUS (Cenarro et al. 2019) and S-PLUS (Mendes de Oliveira et al. 2019) surveys are covering large areas of the northern and southern hemispheres respectively, with 12 filters (including 7 custom-made narrow bands matched to certain key spectral features). Initially conceived to provide an alternative way to calibrate J-PAS data, J-PLUS started in 2017 and is being carried out from a dedicated $80 \mathrm{~cm}$ telescope, the JAST/T80, at the Observatorio Astrofisico de Javalambre (OAJ, Cenarro et al. 2014), located in the province of Teruel, in an exceptionally dark region in continental Spain. J-PLUS has already observed thousands of square degrees and its data are being used for a broad variety of science studies, for instance, the search for metal-poor stars (Whitten et al. 2019b) or the 2D study of nearby galaxies (San Roman et al. 2019) and star forming regions (Logroño-García et al. 2019). Likewise, S-PLUS uses the T80-South (a replica of the JAST/T80) located at CTIO and it has also observed thousands of square degrees with a somewhat different survey strategy than J-PLUS that is optimized to match its science goals (see Mendes de Oliveira et al. 2019).

The Javalambre-Physics of the Accelerating Universe Astrophysical Survey (J-PAS, Benítez et al. 2009, 2014) aims to push this spectro-photometric approach to unprecedented scales by mapping thousands of square degrees using a unique set of 56 filters, 54 partly overlapping narrow bands (NB) with a FWHM of $\sim 145 \AA$, complemented with 2 broader filters in the blue and red extremes of the optical spectrum. Although it is designed and optimized to achieve extremely accurate photometric redshifts that will enable measurements of the cosmological baryonic acoustic oscillation (BAO) signal across a relatively wide range of cosmic epochs, the J-PAS filter system will effectively provide a low-resolution spectrum for every pixel of the sky observed without the target selection biases or fiber collision problems that typically affect spectroscopic surveys. This makes J-PAS a highly versatile project that will produce a unique legacy-value data set for a broad range of astrophysical studies of solar-system bodies, stars, galaxies, and cosmology. The survey will be carried out with the primary telescope at the OAJ, the $2.55 \mathrm{~m}$ Javalambre Survey Telescope (JST/T250), characterized by a very large Field of View ( $3 \mathrm{deg}(D)$. The main instrument of the JST/T250 is the Javalambre Panoramic Camera (JPCam), a 1.2 Gpixel camera spanning an area of $\sim 4.2 \mathrm{deg}^{2}$ with its 14 CCDs. While waiting for the final steps of the assembly and testing of JPCam, the JST/T250 has been equipped with the JPAS-Pathfinder camera, a single CCD camera located at the center of the focal plane, with a field of view of $\sim 0.3 \mathrm{deg}^{2}$. This camera has been used to perform the first scientific operations of the JST/T250 and to deliver, for the first time, the kind of data expected from the full J-PAS survey. A variety of observations have been performed, but most of the effort has been devoted in completing $\sim 1 \mathrm{deg}^{2}$ on the famous Extended Groth Stip (EGS) field, an area covered by many experiments, from AEGIS (Davis et al. 2007) to ALHAMBRA (Moles et al. 2008), CANDELS (Grogin et al. 2011) and the HSC-SSP survey (Aihara et al. 2018, 2019). The JPAS-Pathfinder observations on the EGS field, dubbed miniJPAS, have been used to test the performance of the telescope and to prove the potential of the J-PAS filter system. In this paper, we present the miniJPAS data set and the data products, which are part of the first public release of the J-PAS collaboration. We also provide a flavor of the diverse science cases that can be studied with future J-PAS data.

The paper is organized as follows. Section 2 provides details on the OAJ, the instruments, and filter system and here we describe the miniJPAS, from the observational strategy to basic data statistics. The data reduction and catalog construction pipelines are described in Sect. 3. Section 4 offers an overview of the data products, including photometric redshifts and other value-added catalogs. In Sect. 5, we discuss the quality of the data and in Sect. 6, we highlight some of the main science cases that the J-PAS filter system unlocks. In Sect. 7, we summarize our results.

Throughout this work, we adopt a Lambda Cold Dark Matter ( $\Lambda \mathrm{CDM}$ ) cosmology with $h=0.674, \Omega_{\mathrm{M}}=0.315, \Omega_{\Lambda}=0.685$, as in the latest Planck results (Planck Collaboration VI 2020). Magnitudes are quoted in the AB-system (Oke \& Gunn 1983).

\section{Survey definition and data acquisition}

In this section, we first summarize the technical aspects of the survey and then focus on the data acquisition.

\subsection{Observatorio Astrofísico de Javalambre (OAJ)}

The $\mathrm{OAJ}^{1}$ (Cenarro et al. 2014) is an astronomical infrastructure designed to carry out large-area photometric sky surveys from the northern hemisphere with dedicated, large field-of-view (FoV) telescopes. The definition, design, construction, exploitation, and management of the observatory and the data produced at the OAJ are responsibility of the Centro de Estudios de Física del Cosmos de Aragón $\left(\mathrm{CEFCA}^{2}\right)$, and since 2014 the OAJ has functioned as a Spanish Singular Scientific and Tech-

\footnotetext{
https://oajweb.cefca.es/

http://www.cefca.es
} 


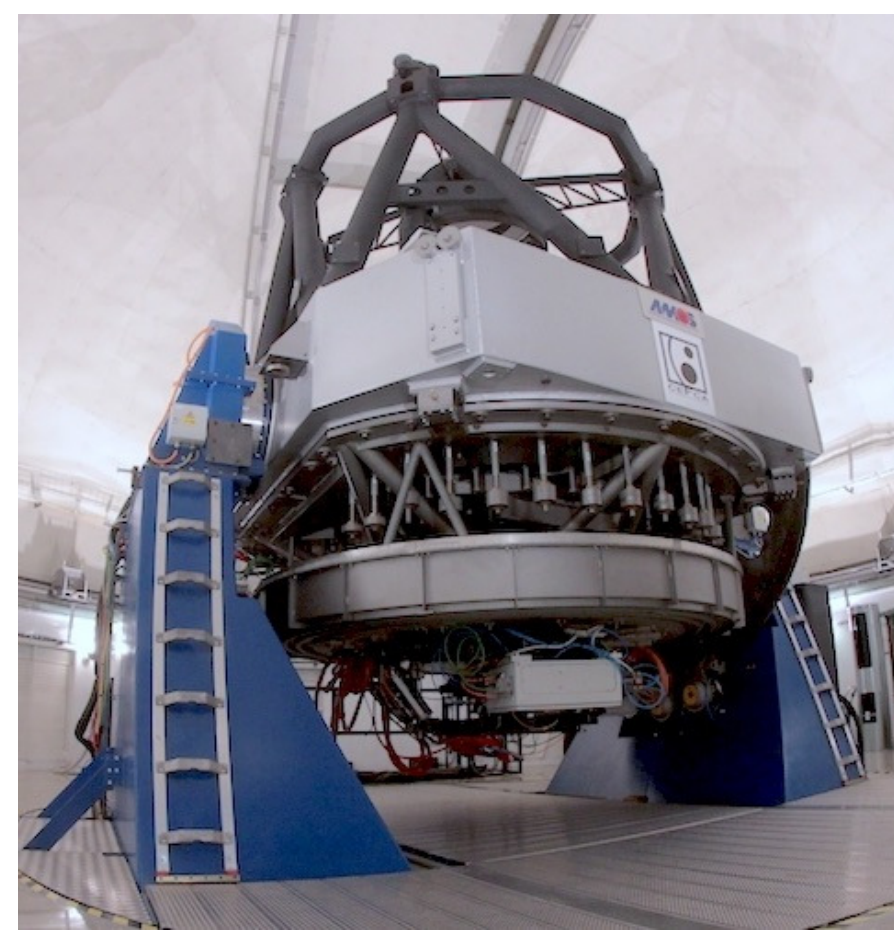

Fig. 1. View of the JST/T250 telescope at the OAJ with the JPASPathfinder camera used to conduct the miniJPAS survey mounted at the Cassegrain focus.

nical Infrastructure (known by the Spanish acronym ICTS for Infraestructura Científico Técnica Singular). The observatory is located at the Pico del Buitre of the Sierra de Javalambre, Teruel, Spain, and is organized around two telescopes, the Javalambre Survey Telescope (JST/T250, Cenarro et al. 2018) and the Javalambre Auxiliary Survey Telescope (JAST/T80), both manufactured by $\mathrm{AMOS}^{3}$. The site, at an altitude of $1957 \mathrm{~m}$, has excellent median seeing $(0.71$ arcsec in $V$ band, with a mode of $0.58 \mathrm{arcsec}$ ) and darkness (typical sky surface brightness of $V \sim 22 \mathrm{mag} \mathrm{arcsec}^{-1}$ ), a feature that is quite exceptional for continental Europe. Full details about the site testing of the OAJ can be found in Moles et al. (2010).

J-PAS will be conducted with the JST/T250 (see Fig. 1), an innovative Ritchey-Chrétien-like, alt-azimuthal, large-étendue telescope, with an aperture of $2.55 \mathrm{~m}$ and a $3 \mathrm{deg}$ diameter FoV. The JST/T250 has a flat focal plane corresponding to a Cassegrain layout. The effective collecting area of JST/T250 is $3.75 \mathrm{~m}^{2}$, yielding an étendue of $26.5 \mathrm{~m}^{2} \mathrm{deg}^{2}$. Motivated by the need of optimizing the etendue, JST/T250 is a very fast

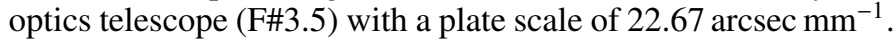
The optical design has been optimized to provide a good image quality in the optical spectral range $(330-1100 \mathrm{~nm})$ all over the $48 \mathrm{~cm}$ diameter focal plane $\left(7 \mathrm{deg}^{2}\right)$. Table 1 illustrates a summary of the main technical characteristics of JST/T250 (see also Cenarro et al. 2018).

The main scientific instrument of the JST/T250, which will be used to carry out J-PAS, is the Javalambre Panoramic Camera (JPCam, Taylor et al. 2014; Marín-Franch et al. 2017), a 1.2 Gpixel camera with an effective FoV of $4.2 \mathrm{deg}^{2}$ and a plate scale of $0.23 \operatorname{arcsec} \mathrm{pix}^{-1}$. JPCam has completed the assembly, integration, and verification phases and is currently being installed and commissioned at the Cassegrain focus of the JST/T250 telescope. Before the arrival of JPCam, however, the

\footnotetext{
https://www . amos.be/
}

Table 1. Main technical characteristics of the JST/T250 telescope.

\begin{tabular}{|c|c|}
\hline Mount: & Altazimuthal \\
\hline Optical configuration: & $\begin{array}{l}\text { Ritchey Chrétien modified, } \\
\text { equipped with a field corrector } \\
\text { and rotator }\end{array}$ \\
\hline M1 diameter: & $2.55 \mathrm{~m}$ \\
\hline Field corrector: & 3 aspherical lenses \\
\hline Effective collecting area: & $3.75 \mathrm{~m}^{2}$ \\
\hline Focus: & Cassegrain \\
\hline F\#: & 3.5 \\
\hline Focal length: & $9098 \mathrm{~mm}$ \\
\hline Plate scale: & $22.67 \operatorname{arcsec} \mathrm{mm}^{-1}$ \\
\hline FoV (diameter) & $3.0 \mathrm{deg}$ \\
\hline Étendue: & $26.5 \mathrm{~m}^{2} \mathrm{deg}^{2}$ \\
\hline EE50 $^{(a)}$ (diameter, as-built) & $12 \mu \mathrm{m}$ \\
\hline EE80 $^{(a)}$ (diameter, as-built) & $25 \mu \mathrm{m}$ \\
\hline
\end{tabular}

Notes. ${ }^{(a)}$ The encircled energy (EE) indicates the fraction of energy contained in a circular aperture of a given radius. In this case, EE50 and EE80 indicate the radius of the circular aperture encompassing the 50\% and $80 \%$ of the energy.

JST/T250 telescope was equipped with its first light instrument, called JPAS-Pathfinder, a single CCD camera built to test the system optical performance and perform the first exploratory scientific operations including the miniJPAS survey.

\subsection{The JPAS- Pathfinder camera}

The JPAS-Pathfinder camera (hereafter, JPAS-PF) is the first scientific instrument installed at the JST/T250 telescope. This single CCD, direct imager was developed with two primary goals in mind: (i) to commission the JPCam Actuator System ${ }^{4}$ and (ii) to start the scientific operation of the JST/T250 while the JPCam was still being assembled in the OAJ clean room.

The JPAS-PF camera is equipped with one large format, $9.2 k \times 9.2 k, 10 \mu \mathrm{m}$ pixel, low noise detector from Teledyne-e2V that is mounted at the center of the JST/T250 FoV. The detector is read out from 16 ports simultaneously. It has an image area of $92.16 \mathrm{~mm} \times 92.32 \mathrm{~mm}$ and a broadband anti-reflective coating for optimized performance from $380 \mathrm{~nm}$ to $850 \mathrm{~nm}$. The JPASPF consists of two main subsystems, the filter and shutter unit (FSU) and the cryogenic camera.

The cryogenic camera is a $1110 \mathrm{~S}$ CCD camera manufactured by Spectral Instruments ${ }^{5}$ (USA). It comprises the scientific detector and its associated controllers, the cooling and vacuum systems, and the image acquisition electronics and control software. The proximity drive electronics provide 16 different operational modes. The miniJPAS survey has been observed with a read mode that achieves total system level noise performance of $3.4 \mathrm{e}^{-}$(rms), allowing for readout times of $12 \mathrm{~s}$ (full frame) and $4.3 \mathrm{~s}(2 \times 2$ binning $)$. The FSU includes a two-curtain shutter provided by Bonn-Shutter $\mathrm{UG}^{6}$ (Germany) that allows us to take integration times as short as $0.1 \mathrm{~s}$ with an illumination uniformity better than $1 \%$ over the entire FoV of the JPAS-PF. The FSU incorporates a single filter wheel designed to integrate

\footnotetext{
4 Because of the large FoV and fast optics, the JST/T250 secondary mirror and the focal plane are actively controlled with two hexapod actuators to ensure optimum image quality.

5 http://www. specinst.com

6 ww. bonn-shutter.de
} 
Table 2. Main technical performances of the JPAS-PF camera.

\begin{tabular}{ll}
\hline \hline CCD format & $9216 \times 9232 \mathrm{pix}$ \\
& $10 \mu \mathrm{m} \mathrm{pix}^{-1}$ \\
Pixel scale & $0.23 \mathrm{arcsec} \mathrm{pix}^{-1}$ \\
Effective FoV & $0.54 \mathrm{deg} \times 0.50 \mathrm{deg}=0.27 \mathrm{deg}^{2}$ \\
Read out time & $12 \mathrm{~s}(\mathrm{full}$ frame $)$ \\
& $4.3 \mathrm{~s}(2 \times 2 \mathrm{binning})$ \\
Readout noise & $3.4 \mathrm{e}^{-} \mathrm{pix}^{-1}$ \\
Full well & $123000 \mathrm{e}^{-}$ \\
CTE & 0.99995 \\
Dark current & $0.0008 \mathrm{e}^{-} \mathrm{pix}^{-1} \mathrm{~s}^{-1}$ \\
Quantum efficiency & $40 \%$ at $350 \mathrm{~nm}$ \\
" & $86 \%$ at $400 \mathrm{~nm}$ \\
" & $93 \%$ at $500 \mathrm{~nm}$ \\
" & $93 \%$ at $650 \mathrm{~nm}$ \\
Filters per filter wheel & 7 \\
\hline
\end{tabular}

seven different J-PAS filters simultaneously. As these filters have a physical dimension slightly smaller than the size of the CCD, the CCD is vignetted on its periphery (see Sect. 3.1). As a result, JPAS-PF provides an effective FoV of $0.27 \mathrm{deg}^{2}$ with a pixel scale of $0.23 \operatorname{arcsec}_{\text {pixel }}{ }^{-1}$. A summary of the technical characteristics of the camera is presented in Table 2.

The JPAS-PF camera was dismounted at the end of 2019 in order to make room for the JPCam.

\subsection{The J-PAS filter system}

The novel and unique aspects of J-PAS lie in its filter system: 54 narrow band filters ranging from $3780 \AA$ to $9100 \AA$, complemented by 2 broader filters at the extreme blue and red ends of the optical spectral range. The NB filters have a FWHM of $145 \AA$ and are spaced by about $100 \AA$ (except for the filter J0378), thus creating a continuous spectral coverage through the entire optical range. The two additional filters are one medium band covering the UV edge (J0348, called $\left.u_{\mathrm{JAVA}}\right)$ and a broad filter red-wards of $9100 \AA$ (J1007). Table 3 lists the main characteristics of the J-PAS filter set, while Fig. 2 shows the transmission curves of the 56 filters described above, where the overall system efficiency has been taken into account (including the atmospheric transmission, the CCD efficiency, and the telescope optics). This filter system effectively delivers a low-resolution $(R \sim 60)^{7}$ spectrum (J-spectra, hereafter) for every object observed. It was specifically designed to achieve a photometric redshift (photo-z) accuracy sufficient for measuring a variety of cosmological probes at different redshifts (see Benítez et al. 2009, 2014, and Sect. 4.2.2). The observations in the 56 filters discussed above are complemented with broad-band observations. The miniJPAS field has been observed with the SDSS-like broad band filters $u_{\mathrm{JPAS}}{ }^{8}, g_{\mathrm{SDSS}}, r_{\mathrm{SDSS}}$, and $i_{\text {SDSS }}$. The $r_{\text {SDSS }}$ filter has been chosen as the detection band for the miniJPAS "dual-mode" catalogs, as explained in Sect. 3.4. From now on, unless otherwise stated, we will refer to these filters simply with $u, g, r$, and $i$. The information on FWHM and central wavelengths of these filters is also avail-

\footnotetext{
7 The wavelength resolution, $R_{\lambda}$, is defined as $R_{\lambda}=\lambda / \Delta \lambda$, so $R \sim 60$ for the J-PAS filter system at intermediate wavelengths.

8 The $u_{\text {JPAS }}$ filter has a redder cut-off than the SDSS $u$, but is broader than the $u_{\mathrm{JAVA}}$.
}

Table 3. Filter system main characteristics.

\begin{tabular}{cccl}
\hline \hline Filter \# & Filter name & $\begin{array}{c}\text { Central } \\
\text { wavelength } \\
{[\AA]}\end{array}$ & $\begin{array}{l}F W H M \\
{[\AA]}\end{array}$ \\
\hline 1 & uJAVA & 3497 & 495 \\
2 & J0378 & 3782 & 155 \\
3 & J0390 & 3904 & 145 \\
4 & J0400 & 3996 & 145 \\
5 & J0410 & 4110 & 145 \\
$\ldots$ & $\ldots$ & $\ldots$ & $\ldots$ \\
54 & J0900 & 9000 & 145 \\
55 & J0910 & 9107 & 145 \\
56 & J1007 & 9316 & High-pass filter \\
\hline
\end{tabular}

Notes. The full table is available in the miniJPAS database in the ADQL table minijpas.Filter.

able, as for the other filters, in the miniJPAS data release ADQL table minijpas.Filter (see Sect. 4.3). The filter transmission curves are publicly available at the Spanish Virtual Observatory page ${ }^{9}$.

Although the definition of the theoretical filter transmission curves was driven exclusively by the main scientific goals of J-PAS, additional functional requirements set by the telescope and instrument opto-mechanical designs were taken into account. The most demanding of these were the physical dimensions of the filters $(101.7 \mathrm{~mm} \times 96.5 \mathrm{~mm})$, central wavelength (CW) uniformity across the filter usable area (the $\mathrm{CW}$ varies by less than $\pm 0.2 \%$ ), a high band-pass transmission (higher than $90 \%$, except for the bluest filters) and flatness (better than $5 \%$ peak-to-valley), out-of-band blocking (OD5 from 250 to $1050 \mathrm{~nm}$ ), and filter-to-filter continuity (overlap at transmissions higher than $75 \%$ ).

The J-PAS filters have been designed by CEFCA and SCHOTT Suisse SA (Switzerland) and manufactured by SCHOTT $^{10}$. A detailed technical description of the filter requirements, design, measurements, and characterization can be found in Marin-Franch et al. (2012), Brauneck et al. (2018a) and Brauneck et al. (2018b).

\section{4. miniJPAS survey observations}

This section is devoted to the description of the miniJPAS observations, including the survey footprint design, observational strategy, and the achieved survey depth and PSF.

\subsubsection{Footprint and survey strategy}

The miniJPAS survey was designed to cover the EGS field. This field was chosen for two main reasons: (i) it is conveniently located in the sky $(\mathrm{RA}, \mathrm{Dec})=\left(+215^{\circ},+53^{\circ}\right)$ for observations at elevations $>30^{\circ}$ from the OAJ during February-July, and (ii) a wealth of multi-wavelength data is available in this region from AEGIS, SDSS, CANDELS and HSC-SSP-Wide. Only four pointings of the JPAS-PF camera (rotated at $45^{\circ}$ with respect to the celestial north) were needed to cover the EGS field. The four pointings comprising the miniJPAS survey of the EGS field are listed in Table 4 and shown in Fig. 3 together with the footprints

\footnotetext{
9 http://svo2.cab.inta-csic.es/theory/fps/index.php? mode $=$ browse\&gname $=0 A$ J

10 wWW. schott. com
} 


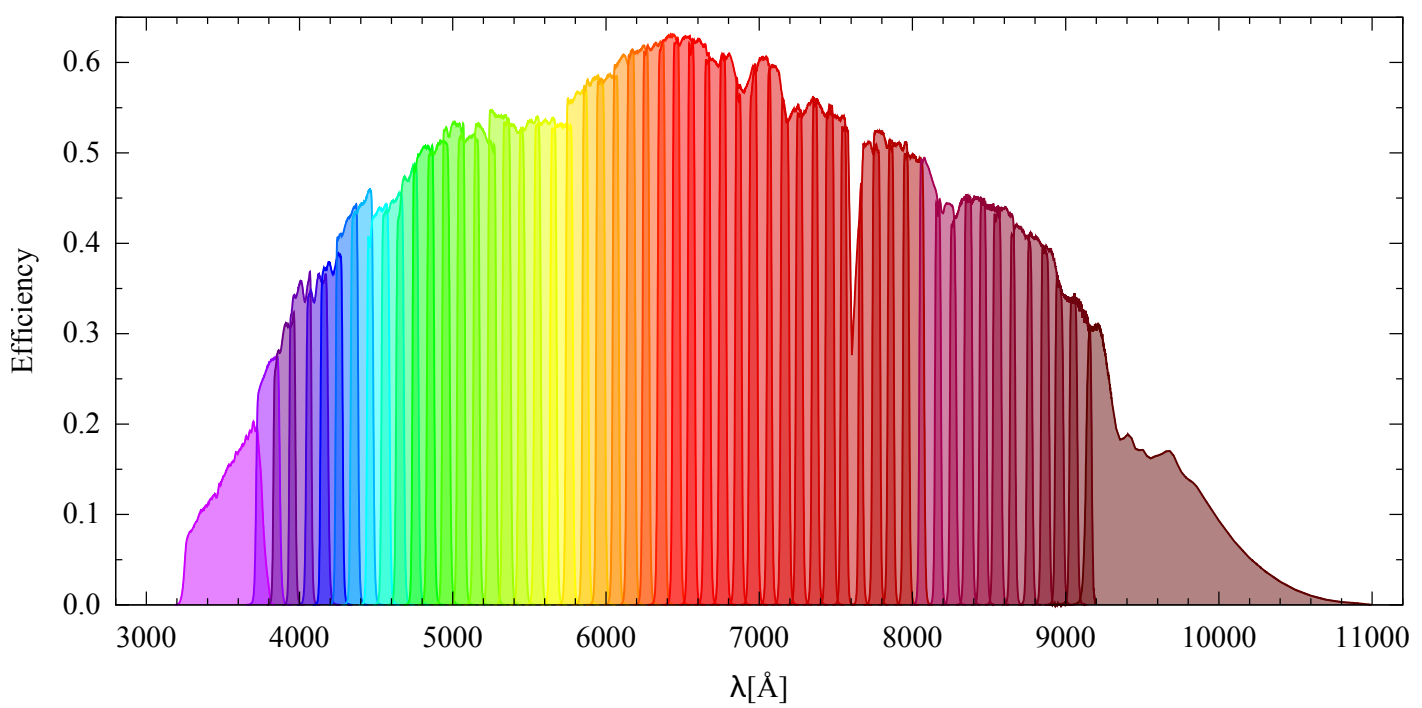

Fig. 2. Measured transmission curves of the J-PAS filters. Effects of the CCD quantum efficiency, the entire optical system of the JST/T250 telescope and sky absorption are included.

Table 4. Central coordinates of each pointing.

\begin{tabular}{lcc}
\hline \hline Tile & RA J2000 (deg) & Dec J2000 (deg) \\
\hline miniJPAS-AEGIS1 & 214.2825 & 52.5143 \\
miniJPAS-AEGIS2 & 214.8285 & 52.8487 \\
miniJPAS-AEGIS3 & 215.3879 & 53.1832 \\
miniJPAS-AEGIS4 & 213.7417 & 52.1770 \\
\hline
\end{tabular}

of several other surveys that have targeted the same region. The four pointings overlap by 3.6 '. Each pointing or "tile" was covered with a minimum of four exposures per filter, dithered by 10 arcsec along the horizontal and vertical directions of the CCD. The total area covered is $\sim 1 \mathrm{deg}^{2}$, while the total area of overlap is $0.09 \mathrm{deg}^{2}$ ( $9 \%$ of the total survey area). After taking the masked areas into account (see Sect. 3.6), the effective area of the miniJPAS survey is $0.895 \mathrm{deg}^{2}$.

In order to maximally benefit from the miniJPAS survey as a J-PAS pathfinder experiment, the miniJPAS survey strategy was dictated by the baseline survey strategy of J-PAS. The latter was described in Benítez et al. (2014) and we summarize it here. Observing times and scanning strategy were defined to deliver the optimal photometric-redshift depth for a broad range of galaxy types and across a wide range of redshifts. The default (single) exposure times assumed are $60 \mathrm{~s}$ for each of the NB filters with four exposures per filter, except for the reddest filter tray which has eight exposes per filter. Due to the technical limitations on the distribution of the filters over the JPCam filter trays, four filters on the blue-side of the spectrum are included in the reddest filter tray, and thus they also have eight exposures. In order to be consistent with J-PAS, the miniJPAS survey has similar exposure times with some modifications dictated by the technical requirements of the JPAS-PF camera. The exposure times were also scaled up with respect to Benítez et al. (2014) to account for the degraded reflectivity of the primary mirror $\mathrm{M} 1{ }^{11}$. In miniJPAS, for the $56 \mathrm{~J}-\mathrm{PAS}$ filters and the $u$ filter each independent exposure was of $120 \mathrm{~s}$, while for the broad bands

\footnotetext{
11 The first re-aluminization of M1 has been performed at the beginning of 2020 together with the integration of JPCam, hence optimizing the telescope performance for the beginning of J-PAS observations.
}

$g, r$ and $i$ each exposure was of $30 \mathrm{~s}$, to avoid saturation. The basic strategy for the narrow bands and $u$ required four exposures, one per dithered position (except for the reddest filters and few blue filters, for which a minimum of eight exposures, two per dithered position, was planned), while the strategy for the broad bands was of 4 exposures per dithered position. Therefore, the total minimum exposure time per filter was set to $480 \mathrm{~s}, 4 \times 120 \mathrm{~s}$ for the NB and $u(960 \mathrm{~s}$ for the reddest filters) and $4 \times 4 \times 30 \mathrm{~s}$ for the broad band (BB). However, for several filters, there are more than this minimum number of images currently available, as multiple observations have been carried out with different sky conditions to test the system.

Concerning the readout modes, the same set-up expected for J-PAS was adopted: $2 \times 2$ binning for the $56 \mathrm{~J}$-PAS filters and full frame mode for $u, g, r, i$. The motivation for the $2 \times 2$ binning in the narrow band filters is to increase the photometric depth reducing the readout noise in the pixels of the binned images by a factor of 4 . The drawback is the under-sampling of the PSF in those images. Finally, we note that the tiling strategy for J-PAS is planned to be different than the one adopted for miniJPAS: for J-PAS the dithering will be of $1 / 2$ of the CCD, so that the same sky area will be observed by different parts of the CCD, mitigating systematic effects due to the small variations of the transmission on the filter position (although this has been demonstrated to be very minor, being the filter's central wavelength uniform across its usable area within $\pm 0,2 \%$ of the central wavelength). For miniJPAS, given its small footprint, we limited the dithering to 10 arcsec, as mentioned above.

\subsubsection{Data acquisition, depth, and FWHM}

The miniJPAS survey data were acquired between May and September 2018, although for few filters, further images were taken in the next available season in 2019 (see Appendix A). Because of the nature of the filter wheel available for the JPASPathfinder camera (see Sect. 2.2), observations were performed in groups of six filters. The filter ordering was chosen to maximize the data validation and scientific analysis as the survey was progressing, and to ensure a broad enough spectral coverage in case some filters could not be observed for unforeseen reasons. Even though the nominal desired minimum elevation of the telescope during observations is $40^{\circ}$, for this first data 

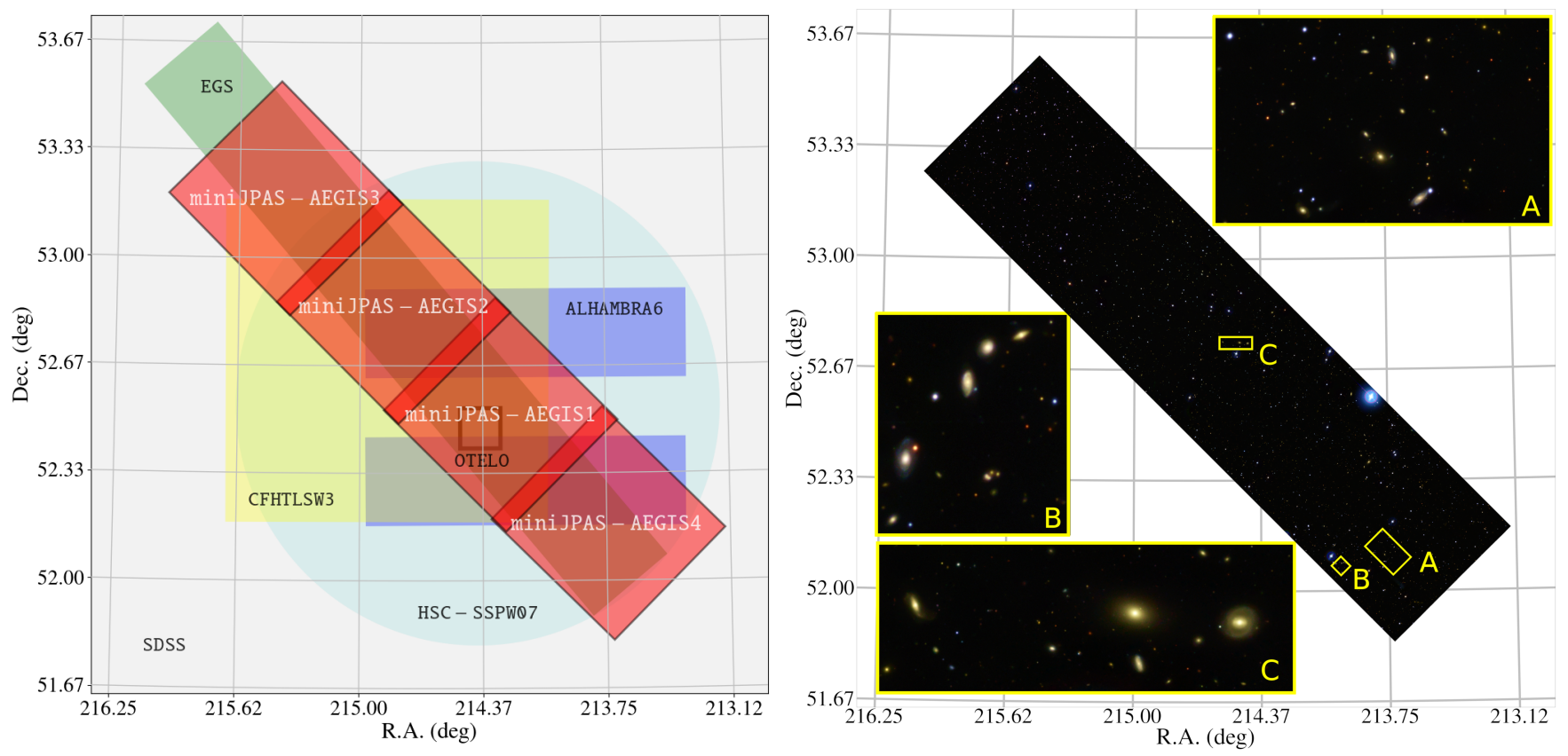

Fig. 3. Left: footprint of the miniJPAS survey, with its four individual pointings shown as red squares (see their coordinates in Table 4). miniJPAS overlaps with the footprints of other projects, also shown for reference: EGS (in green), pointing \#6 of the ALHAMBRA Survey (in violet), the W07 wide field of the HSC-SSP (large circle in pale blue), field W3 of the CFHTLS (in yellow), OSIRIS Tunable Emission Line Object survey (OTELO, http://research.iac.es/proyecto/otelo/pages/otelo.php; small square close to the center of the figure), and SDSS (in light gray occupying the whole area). We refer to Table 9 for the references to these overlapping surveys. Right: $g, r, i$ color image of miniJPAS, with zoom in three selected areas.
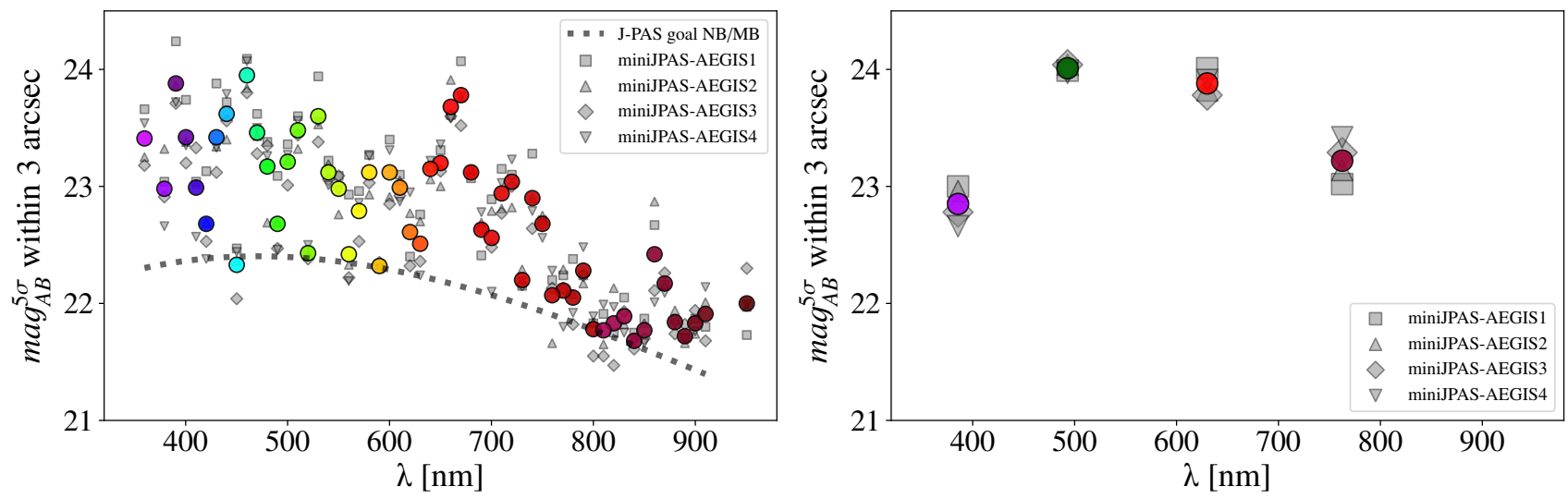

Fig. 4. Image depths ( $5 \sigma$ in a 3 arcsec circular aperture), computed from the noise in each tile for the narrow bands (left) and broad bands (right). The colored symbols indicate the survey average values in each filter, while the gray symbols indicate the values in each of the four miniJPAS pointings. For the narrow bands, the dashed gray line indicates the approximate target minimum depth of J-PAS from Benítez et al. (2014).

set, this restriction was relaxed to allow for observations for a longer period of time. The quality of the data in terms of PSF and depth suffered only mildly from this choice, as images with FWHM below 2 arcsec can be taken down to an elevation of around $30^{\circ}$.

All good-quality exposures were included in the coadded images from which the miniJPAS catalogs were generated (see Sect. 3). The number of exposures per filter and per miniJPAS tile are provided in Appendix A. The resulting depths are shown in Fig. 4. The minimum target depths are reached in all the filters, with most reaching fainter magnitudes than the J-PAS target depths. The filter-to-filter differences in depth depend both on the net sky brightness during the observations and on the final number of coadded exposures. Based on these results and the experience with J-PLUS (Cenarro et al. 2019), the final J-PAS survey strategy will be modified to mitigate these filter-to-filter inhomogeneities in survey depth. We are investigating the possibility of adapting the exposure time for each pointing according to the sky brightness at the time of observing. The final strategy of J-PAS is still in process of fine-tuning, and it will be fully described in a dedicated work (Ederoclite et al., in prep.).

The average FWHM per tile and per filter are shown in Fig. 5. Most of the bands have FWHM below 1.5 arcsec. A slight systematic increase in FWHM can be observed for the reddest bands, from $800 \mathrm{~nm}$ up and, especially, from $860 \mathrm{~nm}$ to higher wavelengths. The main reason for this behaviour is that the reddest filters were scheduled to be observed last, and towards the end of the observing campaign the EGS/AEGIS field reached the lowest elevations. As a consequence, the FWHMs are larger than the ones we would have obtained at lower zenith distances 


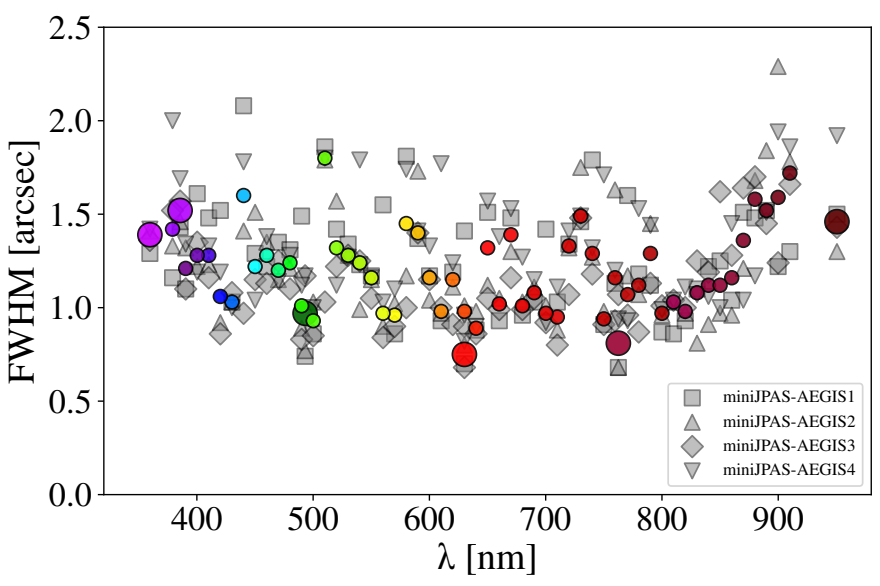

Fig. 5. PSF FWHM over the miniJPAS survey area. The colored symbols represent the survey averages for each filter, while the gray symbols indicate the values found for each of the four miniJPAS pointings. The larger symbols indicate the FWHMs of the broad bands.

with the same atmospheric conditions. Nonetheless, very few tiles have PSFs with FWHM above 2 arcsec. Regarding the 2D stability of the PSF across the images, the median rms FWHM relative variation is $3.7 \%$, when accounting for all filters and tiles, with a normalized median absolute deviation, $\sigma_{\mathrm{NMAD}}$, of $2.3 \%$.

\section{The J-PAS pipeline and data management}

All data collected by the OAJ observatory are handled and processed by the Data Processing and Archiving Unit (hereafter UPAD) group at CEFCA. The UPAD data center (Cristóbal-Hornillos et al. 2014) produces reduced and calibrated data, archives the data, and provides access to the data by the whole scientific community. In this section, we summarize the several data managing steps, from the construction of coadded images to the development of the source catalogs.

\subsection{Processing of single frames}

The detrending of the single frames follows the standard steps of bias subtraction, pre and overscan subtraction, trimming, flat field correction, illumination correction and, if needed, fringing corrections. We refer to Cenarro et al. (2019) for details on how these steps have been implemented, as the data reduction of miniJPAS follows that of J-PLUS. Here, we focus on a few particular aspects of the treatment of miniJPAS data (e.g., reflections and background patterns) due to the fact that the JPAS-PF was not specifically designed for the JST/T250 and its FoV is significantly smaller than that of the telescope ${ }^{12}$. The main issues that needed special treatment were:

Vignetting. The first issue that was detected in the JPAS-PF images is a strong vignetting in the outer parts of the CCD. As a result, the images show a strong gradient of efficiency. To minimize the impact on the final measurements, the images need to be trimmed to exclude regions with low efficiency. The preliminary tests required a reduction of the effective area of the CCD from $9216 \times 9232$ pixels to $7777 \times 8473$. The resulting effective FoV of JPAS-PF is thus $0.27 \mathrm{deg}^{2}$ (see Table 2).

\footnotetext{
12 We expect that most of these issues will not be relevant for J-PAS images produced by JPCam, because both the camera and the telescope were designed together as a unique optical system.
}

Background patterns. Another important issue detected in the JPAS-PF images are background patterns with strong gradients and variations on time scales of a few minutes which affect different filters in different ways. We were able to identify two different sources of reflection. The first one is characterized by straight patterns and is likely due to the optics of the camera. The second one, instead, features circular patterns affecting many of the reddest filters, and is likely due to the small variations in the central wavelengths of the filters. In both cases, the net effect is an irregular distribution of the background light affecting the images in two ways: it affects the flat field images via spurious changes of efficiency and creating an irregular background pattern. The first is corrected through the illumination correction while the second requires a careful subtraction so as not to affect the photometry of the objects. The technical details of the procedures followed to remove these background patterns can be found in Appendix B. The left panels of Fig. 6 show an example of an image before and after the background subtraction.

Fringing. For filters redder than J0740, the presence of fringing requires an extra data reduction step to remove the pattern. We follow the standard implementation already used for the $z$ filter of J-PLUS and constructed master fringing images using all available images taken with JPAS-PF in each filter. This is possible as the fringing pattern is very stable across nights. However, some small residual fringing can still be seen in the final images for a few filters for which the pattern is particularly strong (e.g., the J1007 filter) and for which the number of available images is small.

The right panels of Fig. 6 show an example of an image taken in the J0880 filter before and after full processing. In the raw image, we can see the vignetting around the edges, which is present in all of the images, as well as the circular background pattern and fringing, which are common in the red filters.

Astrometric calibration is the last step needed for the proper combination of the images. This is carried out as a part of the standard procedure for OAJ images using the software Scamp (Bertin 2010a) and the Gaia DR2 (Brown 2018) as reference catalog. The typical uncertainty of the final astrometry of the single frames relative to Gaia is $\sim 0.035^{\prime \prime}$, which is only a small fraction $(\sim 7-15 \%)$ of the image pixel size $\left(\sim 0.23^{\prime \prime}\right.$ and $\sim 0.46^{\prime \prime}$ for the unbinned and binned images, respectively).

\subsection{Final coadded images}

Once the single frames are cleaned of instrumental effects, they are combined with the Astromatic ${ }^{13}$ software Swarp (Bertin 2010b). All the images are sampled to the fiducial pixel size of the camera (i.e., $0.23 \operatorname{arcsec~pixel}^{-1}$ ), including all the images of the narrow bands that were observed with a binning of $2 \times 2$. We opted for maintaining the original orientations of the frames instead of rotating the coadded images with north pointing up (increasing $Y$ axis) and east toward the left (decreasing $X$ axis). In addition to the combined science images, Swarp also constructs normalized weight images that keep track of, for example, pixel areas where the number of combined images is lower due to dithering or masking. Therefore, the weight image can be considered as a map of the effective exposure time across

\footnotetext{
13 https://wWw.astromatic . net
} 

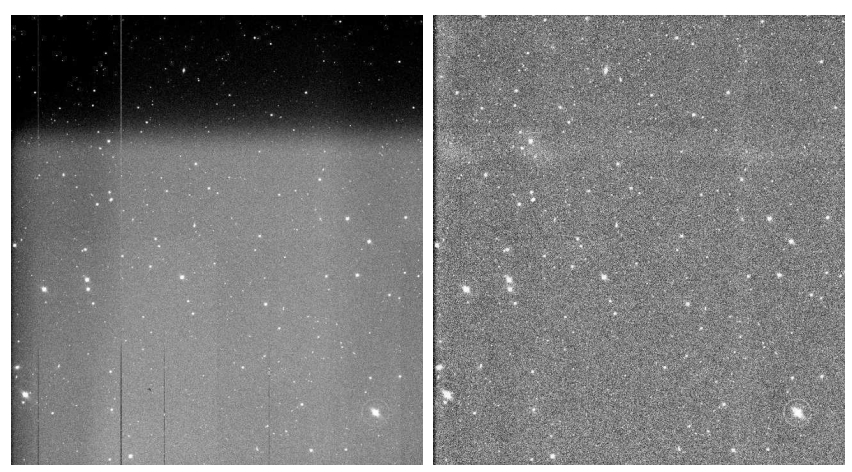

(a)
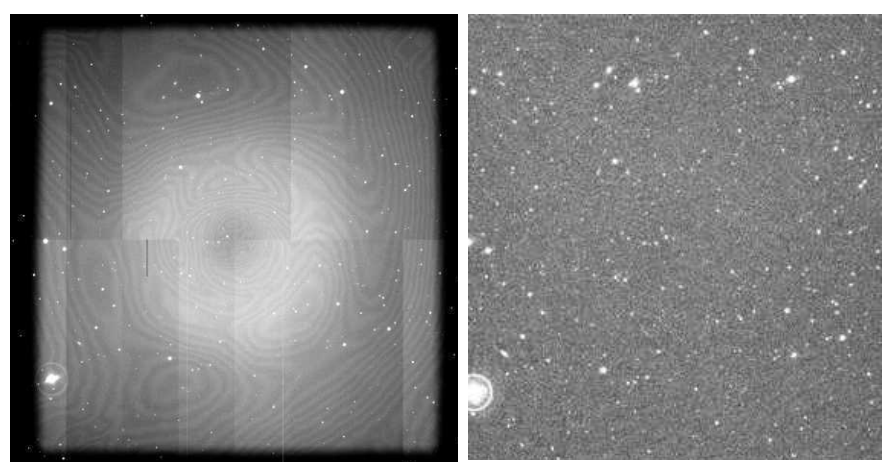

(b)

Fig. 6. Panel a: example of an image before and after background subtraction in the filter J0690 taken from the miniJPAS-AEGIS2 pointing. Panel $b$ : example of an image before and after the full processing in the filter J0880 taken from the miniJPAS-AEGIS1 pointing.

different parts of the image. This is taken into account, for example, in the masking process explained below, in Sect. 3.6.

\subsection{PSF treatment}

One of the main challenges of large field surveys is to provide homogeneous photometry across the large survey area. Each miniJPAS pointing is comprised of 60 filter images with multiple exposures per image. Variations in the PSF from filter to filter may introduce artificial color terms due to light coming from the same area on the sky being distributed differently in the final images corresponding to different filters. These PSF variations will cause problems with the object photometry when the PSF differs significantly between the filter images. miniJPAS uses an image homogenization process to create PSF-matched, aperturecorrected images for consistent photometry measurements, at the expense of degrading some images (see Sect. 3.4). This method uses PSFEx (Bertin 2011) to calculate a PSF homogenization kernel that is used to convert variable instrumental PSFs to constant, round Moffat profiles. The homogenization process introduces some correlated noise across the images. Therefore, the algorithm uses the noise model to recalculate the noise in the images that can then be taking into account when computing photometric errors (Molino et al. 2014). We only apply the homogenization kernel to the corrected PSF apertures (PSFCOR and WORST_PSF, see Sect. 3.4) in the dual-mode catalogs. For all other types of apertures, no such convolution is performed. For each filter image, we provide the overall PSF model produced by PSFEx, and we also provide a service for generating an image of the PSF model at any position of any image (see Sect. 4.1.2).

\subsection{Photometry}

The detection of sources in the images is done with the widely used software SExtractor (Bertin \& Arnouts 1996). To cover as much as possible the needs of the astronomical community we have run SExtractor in two complementary ways:

Dual-mode. To construct catalogs in which the photometry for each objects is carried out self-consistently in the different bands, we ran SExtractor in the so-called "dual-mode". In this mode, SExtractor is first run on a reference ("detection") image for source detection and for the definition of the position and sizes of the apertures. Then it is run on each of the filter images to perform the photrometry within the apertures defined by the reference image (forced photometry). In our case, we chose the $r$ band coadded images as the reference images for constructing the dual-mode catalogs ${ }^{14}$. We note that to compute the different types of magnitudes described in Sect. 4.1, we had to run SExtractor in dual-mode three times, each time with a different treatment of the PSF.

Single-mode. In the so-called "single-mode", both the detection and the photometry are performed for each filter image separately.

The advantage is that objects that are not visible or not detected in the reference image (e.g., faint objects having emission lines in one or more filters but not in the $r$ band) can be identified. A disadvantage is that for the same object the photometry may be done at somewhat different positions or within somewhat different apertures in the different bands, which increases the chance of obtaining inconsistent colors.

The SExtractor detection parameters used were chosen to reach a compromise between large detectability (i.e., completeness), while avoiding spurious detections (i.e., purity). Due to the fact that the noise level in all other bands is higher than that in the $r$ band, the detection threshold (in units of the $\sigma$ of the background) for the generation of the single-mode catalogs was set to a higher value (DETECT_THRESH=2) than for the dual-mode catalogs (DETECT_THRESH $=0.9)^{15}$. The full list of SExtractor parameters can be found in Appendix C. Finally, we note that the miniJPAS photometry was carried out on the images corresponding to each of the four individual miniJPAS pointings. In the regions of overlap between the pointings, the primary catalogs only contain the best detection of each overlapping source (flagged with FLAG $=1024$, see Sect. 4.1.1). However, the alternative measurements for each overlapping source are available in the tables minijpas. Duplicated.

\subsection{Photometric calibration}

The photometric calibration of the miniJPAS survey largely follows the methodology for the calibration of J-PLUS presented in López-Sanjuan et al. (2019a). We applied the following steps:

- Selection of a high-quality sample of stars for calibration. We selected all miniJPAS sources with a $S / N>10$ in all

14 For very faint objects having fluxes close to the noise level, it is possible to obtain negative fluxes in bands different than the detection image. This is not a problem as it is consistent with a measurement that is within the noise.

15 The value of the other parameter controlling the source detection, DETECT_MINAREA, was set to 5 pixels. 
photometric bands and for which a $S / N>3$ Gaia parallax is available. We constructed a diagram of the absolute $G$ magnitude versus $\left(G_{\mathrm{BP}}-G_{\mathrm{RP}}\right)$ color, de-reddened for dust using the 3D dust maps from Green et al. (2018). We then selected those sources belonging to the main sequence, giving 334 calibration stars.

- Calibration of the gri broad-band filters using the PanSTARRS photometry as reference. We compared our 6" aperture magnitudes corrected to total magnitudes with the PSF magnitudes from Pan-STARRS. The aperture corrections were computed from the light growth curves of unsaturated, bright stars in each tile and are stored in the ADQL table mini jpas. TileImage. This step provides the zero points of the gri broad band filters. We also computed the zero points using the J-PLUS photometry as the reference, obtaining differences below $0.01 \mathrm{mag}$.

- Homogenization of the narrow bands with the stellar locus. For each NB, we compute the dust de-reddened $\left(\mathcal{X}_{\text {ins }}-r\right)_{0}$ vs. $(g-i)_{0}$ color-color diagram of the calibration stars, where $X_{\text {ins }}$ is the instrumental magnitude of the selected NB. We then computed the offsets needed for making the stellar locus consistent among the four pointings. This provides a homogeneous instrumental photometry in miniJPAS.

- Absolute color calibration. The last step is to translate the instrumental magnitudes to the magnitudes on top of the atmosphere. In J-PLUS this step was performed using the white dwarf (WD) locus, but there is no high-quality WD locus in the miniJPAS area. We therefore used stellar spectra from BOSS as the reference to provide the absolute calibration of all miniJPAS colors. After a visual inspection of the available BOSS spectra, 40 stars were selected. We compared the synthetic $(\mathcal{X}-r)$ colors from BOSS spectra with the instrumental magnitudes from miniJPAS to obtain one offset per passband except for $r$, which is used as reference and anchored to the Pan-STARRS calibration.

- The BOSS spectra do not cover the $u_{\mathrm{JAVA}}$ and $u_{\mathrm{JPAS}}$ filters. For these bands, we obtained the color offset from a direct comparison with the J-PLUS photometry.

In summary, the absolute flux calibration of miniJPAS was tied to the Pan-STARRS $r$ band fluxes, and the colors were anchored to the BOSS spectra except for the $u_{\mathrm{JAVA}}$ and $u_{\mathrm{JPAS}}$, which were anchored to the J-PLUS photometry.

We also estimated the zero points by direct comparison with J-PLUS photometry in each of the filters that are shared or similar between the two surveys. This comparison shows that our zero points are consistent at the $4 \%$ level. Thus, we conclude that the current photometric calibration has an absolute error of at most $\sim 0.04$ mag. Given the limited number of stellar reference sources that could be used in the calibration, we use this as a safe upper limit when assessing the performance of our flux calibration. Furthermore, we tested the consistency of our calibration procedure by comparing the photometry of the sources in the overlapping regions of the four pointings (see Sect. 5.1). We note that this comparison only reflects uncertainties in the miniJPAS homogenization (step iii) and is not sensitive to the uncertainties in the absolute color calibration (steps iv and v).

For the J-PAS survey, we expect to reach a 1-2\% accuracy in the photometric calibration when an area of $\sim 500 \mathrm{deg}^{2}$ is available. This will provide a few hundred WDs to derive a consistent color calibration with the WD locus and a robust estimation of the uncertainties thanks to a large number of overlapping areas with duplicated measurements of the same sources.

\subsection{Masks}

In order to help the identification of problematic areas in the images, we computed survey masks that are provided in the
MANGLE format ${ }^{16}$ (Swanson et al. 2008). In addition, we flagged all objects falling in those masked areas and stored this information in the FLAGS_MASK column of the catalogs (see Sect. 4.1.1). The problematic areas currently identified and masked are the following:

Window frame mask. This mask identifies regions where the normalized weight map values are less than $85 \%$. This is determined from the tiling weight-map image in order to homogenize the effective exposure times for the same coadd. The threshold value has been selected to be a compromise between maximizing the valid observation area and minimizing regions with a lower effective exposure time (usually as the result of a lower number of images that were combined). Nevertheless, for each object we compute the value of the normalized weight map at its position, which is stored in the parameter NORM_WMAP_VAL. This parameter takes a value of 1 in the area with the highest effective exposure time.

Mask of bright stars. The mask of bright stars discards regions around bright stars found in the Bright Star Catalog ${ }^{17}$ and the Tycho-2 catalog (Høg et al. 2000). The radius of each masked region is a function of the magnitude of the star.

Mask of artefacts. This mask identifies obvious artefacts in the images, usually resulting from light reflections in the telescope or its optical elements. We developed specific algorithms that are able to automatically detect, analyse and mask such artefacts or patterns. In addition, artefacts not detected automatically are manually masked.

We provide the masks for each tile or coadded image as well as a combined mask of the whole miniJPAS footprint ${ }^{18}$.

\section{4. miniJPAS data set and data access}

In this section, we describe the data products available and how to access them.

\subsection{Data products}

This data release includes images, basic catalog data (parameters measured from images, such as photometry or morphology data) as well as value-added information. For the images, we provide not only the final coadded image but also the several ancillary data ${ }^{19}$, such as: (i) The basic parameters of the observations, including total exposure time, photometric zero points, and estimates of the photometric depth; (ii) The information on the single frames used for generating the co-added image, including details on the sky conditions during the observations; (iii) The weight image resulting from the Swarp co-adding procedure (see Sect. 3.2); (iv) The mask (see Sect. 3.6); (v) The PSF model resulting from PSFEx (see Sect. 3.3).

The information related to individual objects or detections is placed in different tables in a relational database. We provide two kinds of tables that store the data resulting from running SExtractor in both "dual-mode" and "single-mode,"

\footnotetext{
${ }^{16}$ https://space.mit.edu/ molly/mangle/

17 https://heasarc.gsfc.nasa.gov/W3Browse/ star-catalog/bsc5p.html

18 The masks are available for download in the "Image Search" service of the Catalogues Web Portal.

19 All this information is available through the "Image Search" service of the Catalogues Web Portal (see Sect. 4.3).

${ }^{20}$ In the database, these tables have in their names the tag Dual and Single, respectively.
} 
Table 5. Numbers of objects detected in each miniJPAS pointing.

\begin{tabular}{lcc}
\hline \hline Pointing & $N_{\text {Dual }}$ & $N_{\text {Single }}$ \\
\hline miniJPAS-AEGIS1 & 20,016 & 167,150 \\
miniJPAS-AEGIS2 & 13,836 & 142,481 \\
miniJPAS-AEGIS3 & 15,792 & 142,496 \\
miniJPAS-AEGIS4 & 14,649 & 152,443 \\
\hline Total & 64293 & 604570 \\
\hline
\end{tabular}

Notes. $N_{\text {Dual }}$ refers to the total number of detections in the dualmode catalogs (see Sect. 3.4 with the SExtractor parameters listed in Appendix C). $N_{\text {Single }}$ refers to the total number of detections in the single-mode catalogs. We note that for the single-mode catalogs a higher SExtractor detection threshold was used (see Sect. 3.4).

explained in Sect. 3.4. For the dual-mode, each detection corresponds to a unique source across all the bands by construction. In the catalogs, for each detection in the reference band, we provide the list of geometrical parameters (e.g., position of the barycentre, shape, and FWHM) as well as different types of photometric measurements in all the other bands. For the single-mode, the relation between measurements in different bands is not so straightforward and requires a strategy for the cross-matching between the different filter catalogs. Therefore, we opted for providing a catalog with all the detections treated independently, leaving it to the user to perform the cross-match in the desired way.

In summary, each entry in the dual-mode catalog corresponds to one object detected in the $r$ band and its photometry in all the J-PAS bands while each entry in the single-mode catalog is a detection of one object in one band (and only if it is detected in that band) and, hence, each detection in each band has its own entry in the single-mode catalog. Table 5 shows a brief summary of the object number counts for each of the four pointings in the dual- and single-mode catalogs.

The catalogs contain photometry measurements given in three different units: (i) $\mathrm{AB}$ magnitudes (names of the tables tagged with MagAB), (ii) flux densities as a function of wavelength in units of $10^{-19} \mathrm{erg} \mathrm{s}^{-1} \mathrm{~cm}^{-2} \AA^{-1}$ (names of the tables tagged with FLambda), (iii) flux densities as a function of frequency in units of $10^{-30} \mathrm{erg} \mathrm{s}^{-1} \mathrm{~cm}^{-2} \mathrm{~Hz}^{-1}$ (names of the tables tagged with Fnu.

Below, we detail the different types of photometry that are provided in the database: ${ }^{21}$

MAG_AUTO, MAG_ISO, MAG_PETRO. These are different types of estimates of total magnitudes. The reader is referred to the SExtractor User's Manual for a detailed description.

MAG_APER_... These correspond to aperture photometry in circular apertures of different sizes. The numbers in the names refer to the sizes of the aperture in arcseconds, for instance, MAG_APER_1_5 corresponds to the photometry in an aperture of 1.5 arcsec in diameter.

MAG_ISO_WORSTPSF, MAG_APER3_WORSTPSF. These correspond to MAG_ISO and MAG_APER_3_Q with the particularity of being measured after transforming all the images of a given pointing to a PSF size equal to the worst PSF among all the images. This is a straightforward procedure for removing the

\footnotetext{
21 For clarity, we use in this description the names of the columns in the tables storing the magnitudes. For tables storing fluxes, the names are equivalent, exchanging MAG with FLUX.
}

effect of PFS variations among different filters on the photometry and, thus, to obtain more robust measurements of the colors. A disadvantage is the loss of information from the images with the better PSF.

MAG_PSFCOR. This photometry is performed following the approach of Molino et al. (2019; see also Molino et al. 2014, 2017) with the aim of applying corrections object by object in the images with worst PSF to correct for the differences in PSF among different bands. To increase the robustness of the color determination, instead of total magnitude estimators such as MAG_AUTO or MAG_ISO, the fluxes are measured within an aperture with the same shape as the Kron aperture and a semimajor axis equal to the KRON_RADIUS. These magnitudes are also called restricted magnitudes (Molino et al. 2017, 2019). Being smaller than the aperture of MAG_AUTO, the signal-to-noise ratio $(\mathrm{S} / \mathrm{N})$ is higher and, therefore, color measurements, which are key in spectral analysis such as photo- $z$ determination, are more robust. We stress, however, that these are not total magnitudes.

\subsubsection{Flags}

In order to easily identify objects with known specific issues, we provide two types of flags are for each detected object.

SExtractor. FLAGS. This parameter is inherited from SExtractor and contains ten flag bits with basic warnings about the source extraction process (see Table 6). The values of 1024 and 2048, respectively, have been added to identify the original eight flags from SExtractor for objects with detections in more than one pointing (in the areas where the pointings overlap) and known variable objects from the cross-match with other surveys. The cataloged value of the FLAGS parameter of a given object is the sum of the individual values of the FLAGS that affect that object.

Mask FLAGS. Besides the issues affecting individual objects, there are large areas of the images that are affected by different problems. Those areas are identified and masked (see Sect. 3.6) and the objects falling inside those masked areas are flagged using the MASK_FLAGS parameter. In Table 7, we list the possible MASK_FLAGS values and their corresponding image issues. As in the case of the FLAGS parameter, the final value of the MASK_FLAGS parameter for each object is the sum of all the individual MASK_FLAGS that applied to that object.

\subsubsection{PSF models}

In Sect. 3.3 we describe how we treated variations of the PSF from filter to filter to achieve a homogeneous photometry. In the database, the PSF models are provided in two different ways: (i) as the direct output of PSFEx. This can be downloaded for each image through the "Image Search" service of the Web Portal; (ii) as FITS images of the actual model of the PSF for a given position in a given image. This is an "on-demand" service accessible via HTTP request to allow for programmed access ${ }^{22}$.

\subsection{Value-added catalogues}

In addition to the basic photometry information described above, the database contains a wealth of ancillary information to facilitate the scientific analysis of the miniJPAS data. Here, we

\footnotetext{
${ }^{22}$ See http://archive. cefca.es/catalogues/minijpas-pdr 201912/download_services.html\#link_get_psf_by_ position.
} 
Table 6. Individual FLAGS values.

\begin{tabular}{|c|c|c|}
\hline Value & Name & Description \\
\hline 1 & Close neighbours & $\begin{array}{l}\text { The object has neighbours, bright and close enough to significantly bias the } \\
\text { photometry, or bad pixels (more than } 10 \% \text { of the integrated area affected) }\end{array}$ \\
\hline 2 & Blending & The object was originally blended with another one \\
\hline 4 & Saturation & At least one pixel of the object is saturated (or very close to saturation) \\
\hline 8 & Truncated & The object is truncated (too close to an image boundary) \\
\hline 16 & Aperture incomplete & Object's aperture data are incomplete or corrupted \\
\hline 32 & Isophotal incomplete & Object's isophotal data are incomplete or corrupted \\
\hline 64 & Memory overflow deblending & A memory overflow occurred during deblending \\
\hline 128 & Memory overflow extraction & A memory overflow occurred during extraction \\
\hline 256 & Not used & \\
\hline 512 & Not used & \\
\hline 1024 & Duplicated & The object has been marked as duplicated \\
\hline 2048 & Known variable & The object could be a known variable \\
\hline
\end{tabular}

Notes. The final value of the FLAGS of a given object will be the sum of all the individual FLAGS values that apply to that object.

describe the most relevant of these additional parameters or products.

\subsubsection{Stellarity index}

The database provides the results of several complementary methods for the star-galaxy classification of the sources, where "stars" are considered all compact, point-like, objects (thus, including quasars and very compact galaxies) and "galaxies" refer to all other resolved objects. We provide three approaches:

SExtractor classification. SExtractor automatically provides a morphological classification (the CLASS_STAR parameter) for all detected objects. We refer to the manual of SExtractor for details on this classification.

Stellar-Galaxy Locus Classification (SGLC). We applied the Bayesian star/galaxy morphological classifier developed in López-Sanjuan et al. (2019b) for J-PLUS data. The concentration versus magnitude diagram presents two distinct populations, corresponding to compact and extended sources (see Fig. 7). We modeled both populations to obtain for each source a probability of being compact or extended, as indicated in Fig. 7 by the color of the symbols. The most relevant update with respect to the J-PLUS methodology is the modification of the galaxy population model. The galaxy locus was assumed to be constant with magnitude in the J-PLUS analysis, but that assumption does not hold at the fainter magnitudes probed by miniJPAS data. Galaxies become apparently smaller at fainter magnitudes, such that the galaxy locus approaches asymptotically the stellar locus. We modelled this trend with an error function calibrated with miniJPAS data. The morphological information from the $g, r$, and $i$ broad band filters was combined. A prior on the $r$ magnitude, accounting for the larger number of galaxies at fainter magnitudes and estimated in each pointing independently, was applied. The modelling of the stellar and galaxy populations was done pointing-by-pointing as in López-Sanjuan et al. (2019b). As we show in Sect. 5.5, the number counts derived from this Bayesian classification agree with the expectations from the literature up to $r \sim 23.5$. The derived probabilities for the morphology of each source are publicly available in the ADQL table minijpas. StarGalclass.

Machine learning classification. We used machine learning (ML) to classify sources of miniJPAS as stars or galaxies in the magnitude interval $15 \leq r \leq 23.5$. In order to
Table 7. Individual MASK_FLAGS values.

\begin{tabular}{lll}
\hline \hline Value & Name & Description \\
\hline 0 & Not mask & Not inside a mask \\
1 & Window & Object is outside the window frame \\
2 & Bright star & Object is bright star or near one \\
4 & Artefact & Object masked due to nearby artefact \\
\hline
\end{tabular}

Notes. The final value of the MASK_FLAGS parameter of a given object will be the sum of all the individual MASK_FLAGS flags that apply to that object.

train and test our classifiers, we cross-matched the miniJPAS dataset with SDSS and HSC-SSP data, whose classification we assume to be trustworthy within the intervals $15 \leq r \leq 18.5$ and $18.5<r \leq 23.5$, respectively. The best ML classifiers are extremely randomized trees (ERT) and random forest (RF), whose performance is shown in Fig. 8 as compared to SExtractor and SGLC, described above. We can see that when using morphological parameters, ERT outperforms SGLC. For the case in which we use only photometric bands, RF is the best classifier. For a more detailed analysis with other methods and metrics, see Baqui et al. (2021). A value-added cata$\log$ is available in the miniJPAS database via the ADQL table minijpas.StarGalClassML.

\subsubsection{Photometric redshifts}

The photometric redshifts (photo- $z$ ) for miniJPAS were obtained with the JPHOTOZ package, developed at CEFCA as part of the reduction pipeline for the J-PLUS and J-PAS surveys. The JPHOTOZ package is a set of python scripts that acts as interface between the database and the actual photo- $z$ computing code, which is a custom version of LEPHARE (Arnouts \& Ilbert 2011) modified to work with a larger number of filters and higher resolution in redshift than typically required for broadband photometry.

LEPHARE computes photo- $z$ by fitting the observed photometry of each source with a set of templates. We used 50 galaxy templates specifically tailored for miniJPAS data. The templates are stellar population synthesis models generated with CIGALE ${ }^{23}$

$\overline{{ }^{23} \text { http://cigale.lam.fr }}$ 


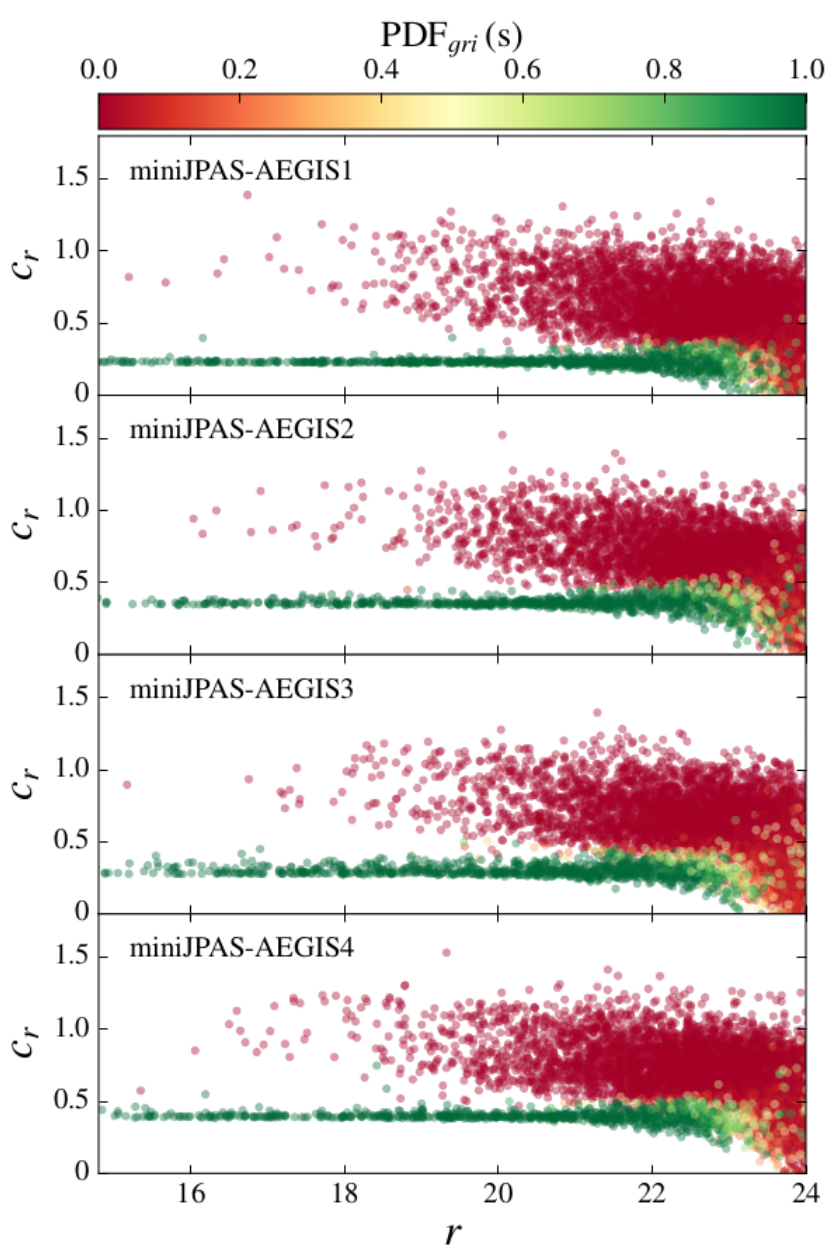

Fig. 7. Concentration-magnitude relation (MAG_AUTO) for sources in each individual miniJPAS pointing. Symbols are color-coded according to the probability of being compact or extended, based on the SGLC of López-Sanjuan et al. (2019b), adapted to the miniJPAS data.

that match the J-spectra of individual miniJPAS galaxies. The process of generation and selection of the most suitable set of templates is described in detail in Hernán-Caballero et al. (in prep.).

To test the accuracy of the miniJPAS photo- $z$ we use a subsample of galaxies with spectroscopic redshifts taken from SDSS DR12 and the DR4 of the DEEP2 Galaxy Redshift Survey (Newman et al. 2013). The latter covers the footprint of the EGS and includes 12051 reliable galaxy redshifts down to magnitude $r=24.1$, with no preselection in magnitude or color. We matched sources in the miniJPAS catalog with those in SDSS and DEEP2 using a search radius of 1.5 arcsec. To ensure a proper evaluation of the photo- $z$ accuracy in galaxies, we considered only sources with a reliable redshift determination $(Q \geq 3$ in DEEP2 or zwarning $=0$ in SDSS $)$ and spectroscopic classification as galaxy. In addition, we excluded sources with FLAGS $>0$ in the miniJPAS photometric catalog. Table 8 summarizes the total number of miniJPAS sources and the number of those used for evaluation of the photo- $z$ accuracy in bins of magnitude.

The error in the photo- $z$ for a given source is expressed by the quantity $\Delta z=\left(z_{\text {phot }}-z_{\text {spec }}\right) /\left(1+z_{\text {spec }}\right)$. The distribution of $\Delta z$ is approximately Gaussian but with heavier wings far from the core due to an almost flat distribution of outliers (defined as those galaxies with catastrophic redshift errors $|\Delta z|>0.05)$ in

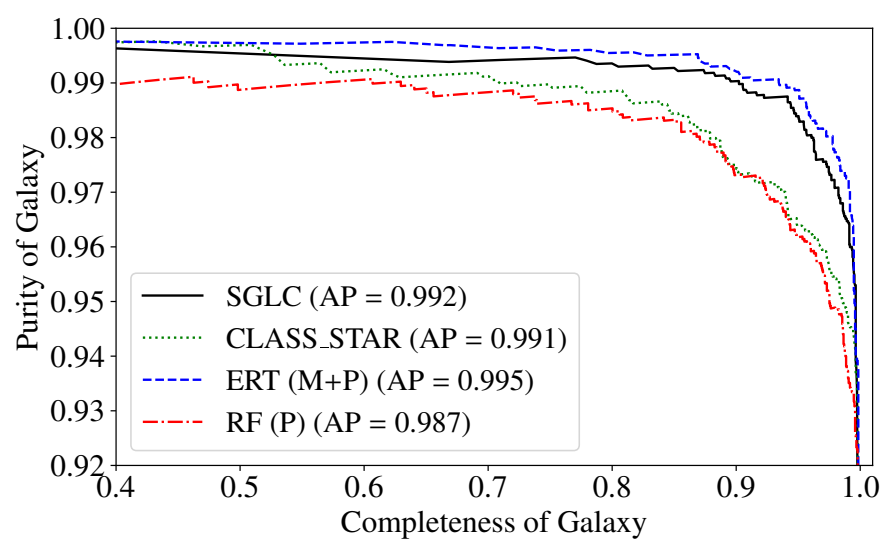

Fig. 8. Purity-completeness curves for galaxies in the magnitude range of $15 \leq r \leq 23.5$, based on the morphological classification from SExtractor (CLASS_STAR, green dotted line), SGLC (black solid line), Extremely randomized trees (blue dashed line) and random forest (red dot-dashed line). ERT uses both the photometric $(P)$ and morphological $(M)$ information of the sources, while the RF uses photometric information only. AP is the area under the purity curve.

the redshift search range. A common statistic used to represent the width of the distribution is the normalized median absolute deviation $\sigma_{\text {NMAD }}=1.4826 \times \operatorname{median}(|\Delta z-\operatorname{median}(\Delta z)|)$, which equals the standard deviation for a purely Gaussian distribution but is less sensitive to the outliers.

It is possible to select samples with more accurate photo$z$ (both in terms of $\sigma_{\mathrm{NMAD}}$ and outlier rate) by sacrificing the sources with the least reliable estimates. Our confidence in the photo- $z$ determination of individual sources depends on the shape of their redshift probability distribution function (PDF). A common parametrization of this confidence is the ODDS parameter (Benítez 2000), defined as the integral of the PDF in a window of fixed width centred at the mode of the PDF. For miniJPAS, we choose a half-width of $0.03(1+z)$ for the integration window. The value of the ODDS ranges from 0 to 1 , with higher values implying higher confidence in the photo- $z$.

In Fig. 9, we show the photometric versus spectroscopic redshift plane for the sources at $r<22.5$, with ODDS $>0.5$, which qualitatively indicates the high level of precision of our photo$z$ solutions. The redshift precision for this sample is $\sigma_{\mathrm{NMAD}}=$ 0.004 and the outlier rate $\sim 4 \%$.

In Fig. 10, we show the $\sigma_{\mathrm{NMAD}}$ and outlier rate for the sample with spectroscopic counterparts as a function of the limiting magnitude in the $r$ band, after applying cuts in ODDS corresponding to completeness of $20,50,80$, and $100 \%$. As expected, both $\sigma_{\text {NMAD }}$ and the outlier rate increase for fainter magnitude cuts due to the decrease in the average $\mathrm{S} / \mathrm{N}$ of the J-spectra. For the entire sample of 2421 galaxies at $r<22.5$, with reliable spectroscopic redshifts, we obtain $\sigma_{\mathrm{NMAD}}=0.0078 \pm 0.0004$ and an outlier rate of $15.3 \pm 8 \%$ (for $\Delta z=0.05$ ), where the uncertainties are calculated using bootstrap resampling. However, it is possible to select subsamples with $\sigma_{\text {NMAD }}$ as low as $\sim 0.002$ and an outlier rate of $\sim 1-2 \%$ by imposing a more restrictive cut in ODDS. We note that further refinement of the photometry might lead to even better photo- $z$ depth. The threshold value of the ODDS parameter required to achieve the desired redshift accuracy or completeness is shown in Fig. 11. In Sect. 6.4 we show how different cuts in ODDS translate to cuts in number densities and redshift precision for sources at different redshifts. 
Table 8. Redshift precision, in units of $\left[10^{-2}\right]$ for different magnitude cuts, differential and cumulative, and for different completeness cuts (100\%, $80 \%, 50 \%$, and $20 \%$ ).

\begin{tabular}{lllllll}
\hline \hline$r$ (MAG_AUT0) & Ntot $^{(a)}$ & Nused $^{(b)}$ & $\sigma_{\text {NMAD }}\left[10^{-2}\right](100 \%)$ & $\sigma_{\text {NMAD }}\left[10^{-2}\right](80 \%)$ & $\sigma_{\text {NMAD }}\left[10^{-2}\right](50 \%)$ & $\sigma_{\text {NMAD }}\left[10^{-2}\right](20 \%)$ \\
\hline $20.0-20.5$ & 1103 & 155 & $0.34 \pm 0.04$ & $0.28 \pm 0.03$ & $0.25 \pm 0.04$ & $0.20 \pm 0.04$ \\
$20.5-21.0$ & 1633 & 226 & $0.43 \pm 0.04$ & $0.39 \pm 0.04$ & $0.34 \pm 0.05$ & $0.26 \pm 0.04$ \\
$21.0-21.5$ & 2360 & 394 & $0.68 \pm 0.06$ & $0.58 \pm 0.07$ & $0.40 \pm 0.04$ & $0.33 \pm 0.04$ \\
$21.5-22.0$ & 3404 & 645 & $1.21 \pm 0.10$ & $0.90 \pm 0.10$ & $0.67 \pm 0.06$ & $0.41 \pm 0.08$ \\
$22.0-22.5$ & 4795 & 773 & $2.30 \pm 0.29$ & $1.72 \pm 0.20$ & $1.11 \pm 0.12$ & $0.58 \pm 0.07$ \\
$22.5-23.0$ & 6972 & 935 & $4.56 \pm 0.34$ & $3.74 \pm 0.37$ & $2.39 \pm 0.38$ & $1.30 \pm 0.17$ \\
\hline$r$ (MAG_AUT0) & Ntot $^{(a)}$ & Nused $^{(b)}$ & $\sigma_{\text {NMAD }}\left[10^{-2}\right](100 \%)$ & $\sigma_{\text {NMAD }}\left[10^{-2}\right](80 \%)$ & $\sigma_{\text {NMAD }}\left[10^{-2}\right](50 \%)$ & $\sigma_{\text {NMAD }}\left[10^{-2}\right](20 \%)$ \\
\hline$<20.5$ & 4016 & 383 & $0.26 \pm 0.02$ & $0.23 \pm 0.02$ & $0.21 \pm 0.02$ & $0.21 \pm 0.02$ \\
$<21.0$ & 5649 & 609 & $0.31 \pm 0.02$ & $0.28 \pm 0.02$ & $0.23 \pm 0.02$ & $0.22 \pm 0.02$ \\
$<21.5$ & 8009 & 1003 & $0.40 \pm 0.02$ & $0.34 \pm 0.02$ & $0.25 \pm 0.02$ & $0.20 \pm 0.02$ \\
$<22.0$ & 11413 & 1648 & $0.56 \pm 0.02$ & $0.45 \pm 0.03$ & $0.32 \pm 0.02$ & $0.22 \pm 0.02$ \\
$<22.5$ & 16208 & 2421 & $0.78 \pm 0.04$ & $0.59 \pm 0.02$ & $0.39 \pm 0.02$ & $0.24 \pm 0.02$ \\
$<23.0$ & 23180 & 3356 & $1.21 \pm 0.05$ & $0.83 \pm 0.04$ & $0.51 \pm 0.02$ & $0.28 \pm 0.02$ \\
\hline
\end{tabular}

Notes. ${ }^{(a)}$ Total number of objects (including compact sources) in miniJPAS for each magnitude bin/cut. ${ }^{(b)}$ Number of objects with spectroscopic counterpart used to calculate the $\sigma_{\mathrm{NMAD}}$ statistics for each magnitude bin/cut. The sub-sample is selected imposing no flags in miniJPAS (flags $=0$ ) in all bands, $0<z_{\text {spec }}<1.5$ and flags $z Q \geq 3$ for DEEP2 and zwarning $=0$ for SDSS.

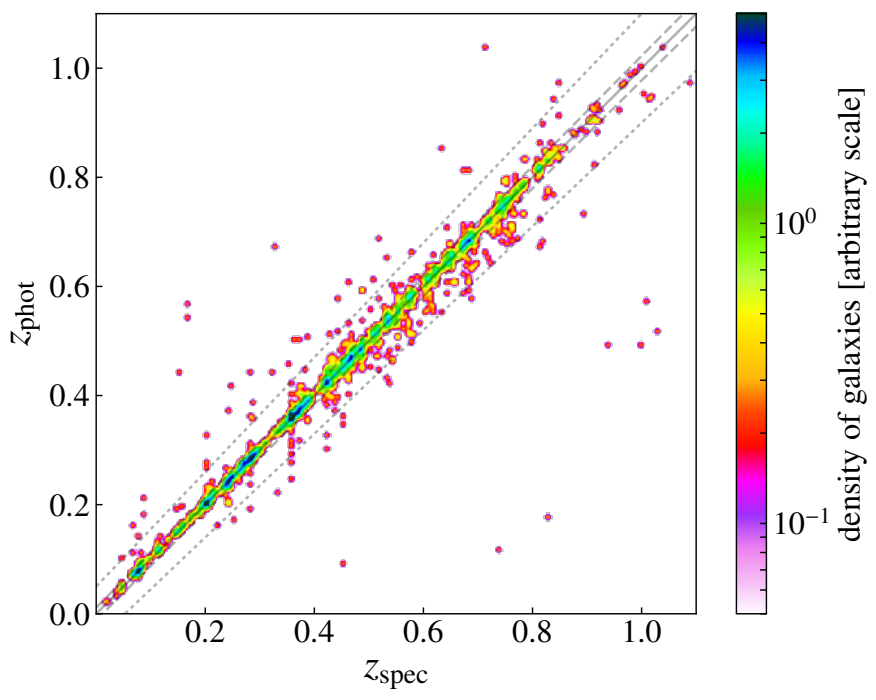

Fig. 9. Comparison between photometric and spectroscopic redshifts for miniJPAS galaxies at $r<22.5$ with ODDS $>0.5$. In order to better highlight the density of points, we have applied a 2D Gaussian smoothing to the data. The solid line marks the 1:1 relation. The two dashed lines enclose a region containing $80 \%$ of the sources, while the dotted lines indicate the $|\Delta z|=0.05$ threshold used to define redshift outliers. We note that each outlier smoothed region corresponds to an individual source.

The accuracy of the photo- $z$ for galaxies also depends on their spectral class. In particular, luminous red galaxies (LRGs) are expected to have more accurate photo- $z$ compared to the general population at the same magnitude thanks to a strong $4000 \AA$ spectral break. To estimate the photo- $z$ accuracy for LRGs we split miniJPAS galaxies in two samples according to the starforming or quiescent classification based on SED-fitting, discussed in Sect. 6.3, which has been performed for galaxies with $r<22.5$. This classification is broadly consistent with a red or blue classification based on the $\mathrm{Dn}(4000)$ index, which measures the strength of the $4000 \AA$ break (see Balogh et al. 1999). Figure 12 shows the redshift accuracy as a function of the limiting magnitude for the subsamples of passively evolving and main sequence, star-forming, galaxies. We note that the

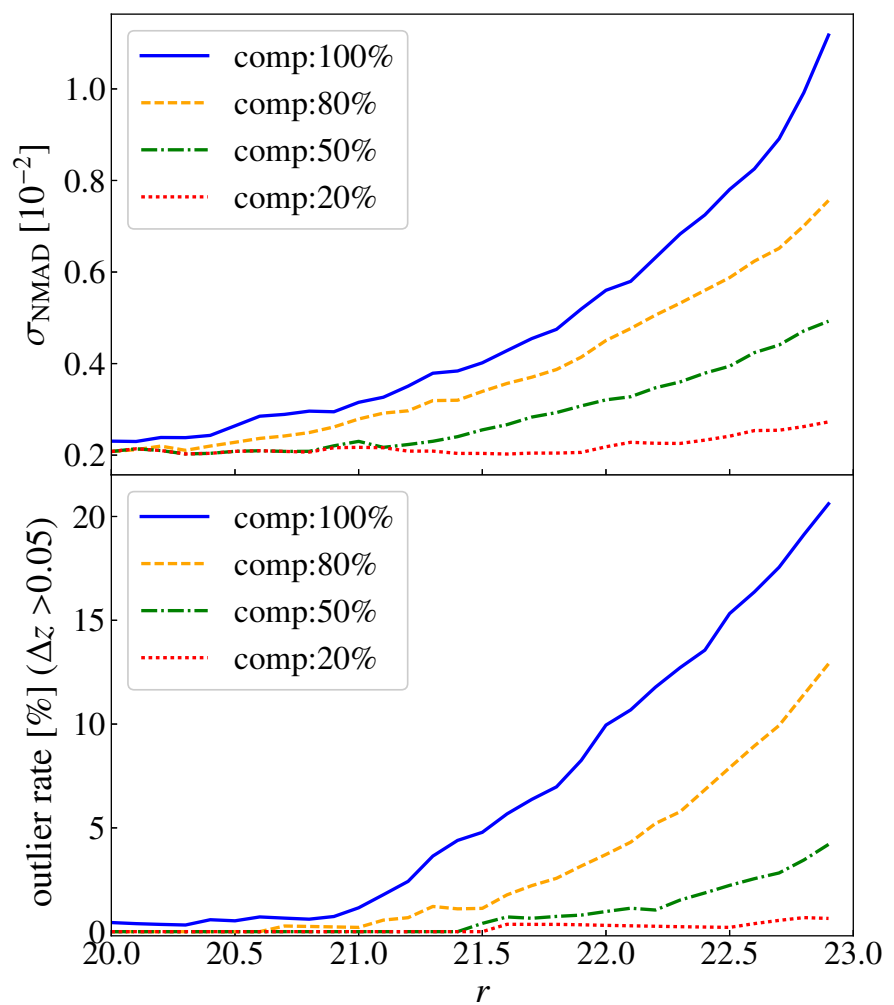

Fig. 10. Normalized median absolute deviation of photo- $z$ errors (top panel, in units of $\left[10^{-2}\right]$ ) and outlier rate (bottom panel) as a function of the limiting magnitude in the $r$-band (MAG_AUTO). Outliers are defined as having redshift errors $|\Delta z|>0.05$. The colors refer to different cuts in the ODDS parameter, resulting in the selection of $100,80,50$ or $20 \%$ of the sources in the spectroscopic sample. The $\sigma_{\mathrm{NMAD}}$ values and the associated errors are provided in Table 8.

redshift precision of blue galaxies depends more strongly on the magnitude. This is because at fainter magnitudes, the emission lines typical of blue star-forming galaxies become weaker and we thus have to rely on the continuum to estimate their photo- $z$. For passive galaxies, instead, the precision of the photo- $z$ estimate decreases only weakly with magnitude. This same classification and the results on redshift accuracy are used in Sect. 6.4 


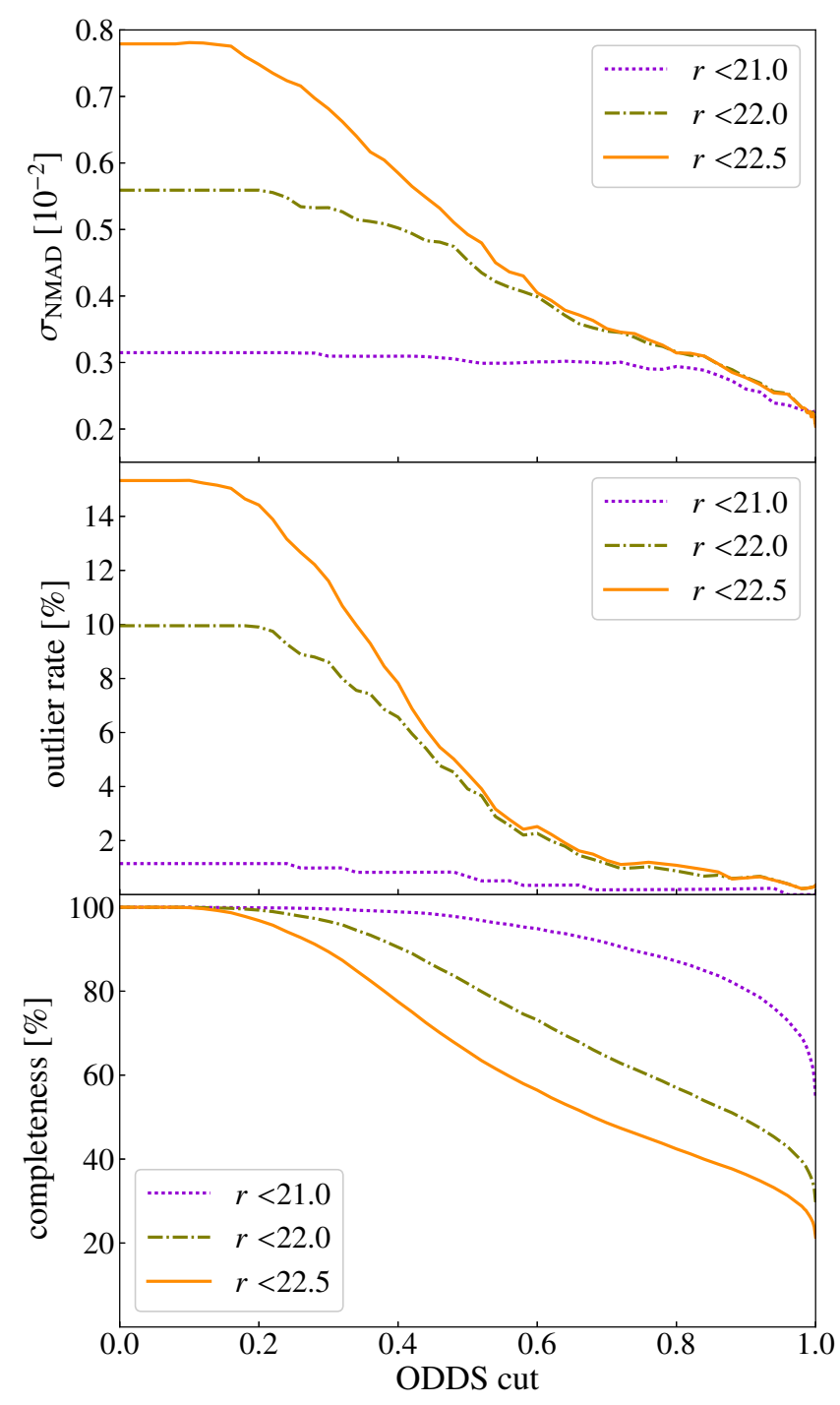

Fig. 11. Dependence of the Normalized median absolute deviation of photo- $z$ errors $\left(\sigma_{\mathrm{NMAD}}\right)$ (top panel), outlier rate (middle panel), and completeness (bottom panel) on the ODDS parameter for different $r$ band cuts.

to discuss the derived number densities of red and blue galaxies, in view of clustering studies of the large-scale structure.

\subsubsection{Extinction and cross-matches}

We provide an estimate of the integrated $E(B-V)$ color excess due to the Milky Way extinction along the line of sight of each detected source in the database table minijpas. MWExtinction. This was derived from the Bayestar17 (Green et al. 2018) dust maps and coupled with the extinction coefficients $k_{\lambda}$ gathered in table minijpas.Filter and computed from the Schlafly et al. (2016) extinction curve following the prescription in Whitten et al. (2019b). In addition to the $E(B-V)$ value, we also provide a precomputed extinction correction $k_{\lambda} E(B-V)$ for each source.

Finally, we provide a cross-match with the surveys listed in Table 9. For catalogs with a Vizier ID, the cross-match has been performed using the CDS X-Match Service ${ }^{24}$. We note that we are reporting, for each miniJPAS source, all the objects within

\footnotetext{
${ }^{24}$ http://cdsxmatch.u-strasbg.fr/
}

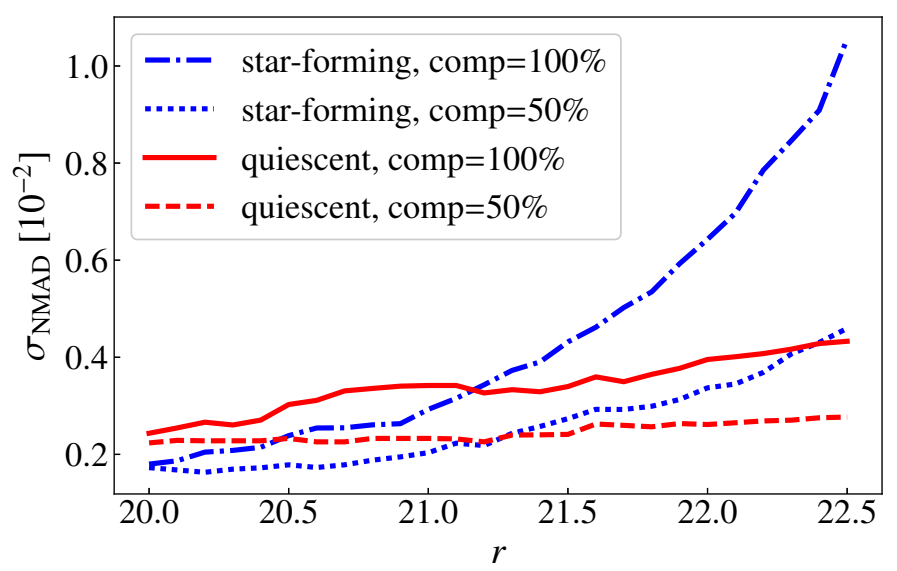

Fig. 12. Dependence of photo- $z$ errors on the limiting magnitude in the $r$-band for galaxies in the "passive" subsample (red line) compared to other galaxies (main sequence and starburst galaxies, blue line). Solid lines indicate values for $100 \%$ completeness (no cut in ODDS), while dashed lines correspond to the 50\% with highest ODDS in each magnitude bin.

the search radius. Therefore, a miniJPAS source may have multiple counterparts in the cross-match catalogs.

\subsection{Data access}

Large projects such as J-PAS require an easy and agile access to the data. For this purpose, CEFCA has developed a powerful Science Web Portal ${ }^{25}$ offering advanced tools for data search, visualization and download, each suited to a particular need (Civera \& Hernandez 2020). The data of this Public Data Release (PDR-201912) of miniJPAS can be accessed online ${ }^{26}$.

The portal includes a user-friendly sky navigator service including color images of the survey. Sources are highlighted, and clicking on an object allows the user to visualize a summary of its properties and its J-spectrum. Further object exploration is possible through an object visualization tool. This tool displays the detailed information about the selected source and the image thumbnail in each filter. It also provides tools for downloading reports and custom object fits cutouts as well as to perform cross matches with other catalogs.

An object list search tool is also offered, which lets the user upload a list of sky positions, object names, or object identifiers and then returns a list of objects near those positions, displaying a customizable summary, J-spectra and thumbnail images for all objects returned. Users can also retrieve a list of objects within a certain angular distance to a given sky position, optionally with additional brightness and photometric redshift criteria, using the cone search service.

To download the coadded images and derived products, an image search service has been implemented where users can select and download the desired data using a variety of search criteria. A coverage map service is also available which helps users to visualise the sky area covered and the distribution of the pointings of the survey. With the multi-order coverage maps (MOC) download service (Fernique et al. 2019), users can download the MOC file which describes the area covered by the survey, which can then be used to compute very fast data set operations (e.g., unions and intersections) or query for data

\footnotetext{
25 http://archive.cefca.es/catalogues

26 http://archive.cefca.es/catalogues/ minijpas-pdr201912
} 
Table 9. External catalogs for which a precomputed cross-match with miniJPAS is provided.

\begin{tabular}{lllll}
\hline \hline Catalogue name & Reference & Vizier ID & $\begin{array}{l}\text { Search } \\
\text { radius }\end{array}$ & Data base table \\
\hline J-PAS DR1 & Cenarro et al. (2019) & - & $3^{\prime \prime}$ & xmatch_jplus_dr1 \\
ALHAMBRA Survey & Molino et al. (2014) & J/MNRAS/441/2891/photom & $4^{\prime \prime}$ & xmatch_alhambra \\
Gaia DR2 & Brown (2018) & I/345/gaia2 & $1.5^{\prime \prime}$ & xmatch_gaia_dr2 \\
PanSTARRS DR1 & Chambers et al. (2016) & II/349/ps1 & $1.5^{\prime \prime}$ & xmatch_panstarrs_dr1 \\
SDSS DR12 & Alam et al. (2015) & V/147/sdss12 & $1.5^{\prime \prime}$ & xmatch_sdss_dr12 \\
All WISE & Cutri et al. (2014) & II/328/allwise & $4^{\prime \prime}$ & xmatch_allwise \\
GALEX AIS & Bianchi et al. (2011) & II/312/ais & $4^{\prime \prime}$ & xmatch_galex_ais \\
CFRS & Lilly et al. (1995) & VII/225B/catalog & $4^{\prime \prime}$ & xmatch_cfrs \\
DEEP2 & Coil et al. (2004) & II/301/catalog & $1.5^{\prime \prime}$ & xmatch_deep2_photo \\
DEEP2 All & Matthews et al. (2013) & III/268/deep2all & $1.5^{\prime \prime}$ & xmatch_deep2_spec \\
HSC-SSP PDR2 & Aihara et al. (2019) & - & $1.5^{\prime \prime}$ & xmatch_subaru_pdr2 \\
\hline
\end{tabular}

(e.g., sources or images) using external tools, such as VizieR ${ }^{27}$, Aladin ${ }^{28}$ or Topcat ${ }^{29}$.

An asynchronous queries interface based on the Virtual Observatory (VO) ${ }^{30}$ Table Access Protocol (TAP, Dowler et al. 2010) has also been implemented in the portal where users can perform Astronomical Data Query Language (ADQL) queries (Osuna et al. 2008) directly on the database. All of these services support the Simple Application Messaging Protocol (SAMP, Boch et al. 2012) that enables the catalogues portal to interoperate and communicate with VO-compatible applications ${ }^{31}$.

Catalogue data and images are also accessible through VO protocols (Hernandez et al. 2020). These VO services allow users to access data in a standardized way using VO compatible applications or their own scripts. Images are available via the Simple Image Access Protocol (SIAP, Dowler et al. 2015) that allows not only for a search of all images covering a sky region, but also for the fits images, cutouts, or color images to be retrived. Catalogue data is accessible both via Simple Cone Search (SCS, Plante et al. 2008) and TAP. The first allows us to search all the objects within a given radius around a specified location, while TAP offers a more flexible access to data tables allowing for complex searches (using ADQL) to be undertaken on the tables storing information about the images, filters, objects, and photo- $z$. Below, we provide a summary of the different tools available to access the miniJPAS data:

Sky Navigator. Sky exploration by panning and zooming. By clicking on an object, we obtain a summary of its properties and options to explore it further or to search for it in other catalogues.

Object List Search. Search for a list of objects via sky positions, object names, or object identifiers. It returns a list of sources near those positions and displays a summary, photospectra, and thumbnail images for the list of objects. name.

Image search. Search and download images by position or

Cone search. Search the database for objects near a certain sky position. Restrictions on colors, magnitudes, and photo- $z$ can be added.

\footnotetext{
27 http://vizier.u-strasbg.fr/

${ }^{28}$ https://aladin.u-strasbg.fr/aladin.gml

${ }^{29}$ http://www.star.bris.ac.uk/ mbt/topcat/

${ }^{30}$ http://www.ivoa.net/

31 http://www . ivoa.net/astronomers/applications.html
}

Coverage map. Shows the sky area covered by the data release. The map is linked to the Sky Navigator.

Multi-Order Coverage Map (MOC). To download the MultiOrder Coverage map (MOC), which describes the area covered by the data release (FITS file).

VO Services. Access to images and objects data through the Virtual Observatory (VO) protocols using VO compatible applications. VO services offered are Simple Cone Search (SCS), Table Access Protocol (TAP), and Simple Image Access Protocol (SIAP).

VO Asynchronous Queries (ADQL). Search the database via Astronomical Data Query Language (ADQL) queries. A help manual with examples is provided.

Direct Download Services. Allow easy automatic access to most of the data. It is possible to download some data directly through simple HTTP access ${ }^{32}$. The data currently available through this service include:

- Full images and weight maps in FITS format and PNG

- "On-demand" cutouts of images and weight maps in FITS and PNG formats

- Masks of individual images in MANGLE format

- PSF models of full frames in PSFEx format or as FITS images of the PSF models at a given position in any image

- "On-demand" PSF models for a given position and image in FITS format

- Photo- $z$ catalogs

- Information on the original individual images

\section{Data quality}

In this section, we show the results of a variety of tests aimed at characterizing the quality of the data.

\subsection{Homogeneity across the footprint}

A powerful test of the consistency of the data reduction and photometric calibration is the comparison of the photometry of objects in the overlap area of adjacent pointings. For these objects, the data reduction and calibration are performed twice, in an independent way in each pointing (see Sect. 3.5). Based on

\footnotetext{
32 http://archive.cefca.es/catalogues/ minijpas-pdr201912/download_services.html
} 


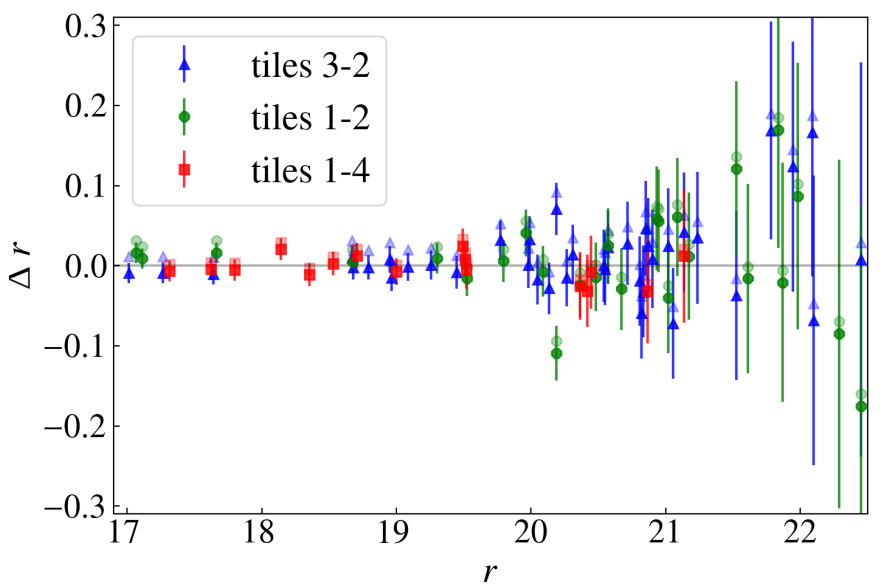

Fig. 13. Magnitude differences measured for objects found in the overlapping pointings using a fixed aperture of 6 arcsec in the $r$ band (rSDSS). Each point corresponds to a point source in one of the overlapping areas (distinguished here by the colors). The light symbols are the magnitude differences before correcting for the PSF of each tile, and the heavy symbols are the magnitude differences after that correction is applied. The error bars are those of the individual tiles, added in quadrature.

the miniJPAS observation strategy, the overlap area is approximately $10 \%$ of the total area of the footprint (see Sect. 2.4.1).

We compared the magnitudes of point sources (CLASS_STAR >0.5) within a 6 arcsec aperture, taking into account the corrections to the flux driven by the variations of the PSF between the different observations (i.e., the light profile of the point sources was used to extend the flux beyond the cut-off at 6 arcsec). The result of that comparison is shown in Fig. 13 for the $r$ band, where we plot the difference in the magnitudes for each object in the overlap area of two contiguous tiles, $\Delta r=r_{a}-r_{b}$, as a function of the mean magnitude, $r=\left(r_{a}+r_{b}\right) / 2$, with error bars given by $\sigma=\left(\sigma_{a}^{2}+\sigma_{b}^{2}\right)^{1 / 2}$, where $(a, b)=(1,2),(1,4),(2,3)$ (numbers correspond to the four miniJPAS pointings). In the figure, we show the differences in magnitudes before (light symbols) and after (heavy symbols) the PSF correction. From the scatter of the data points we can derive an approximate estimate of the calibration error for each filter. For this, we estimate the systematic error that needs to be added to the statistical errors to obtain a reduced $\chi^{2}$ of approximately unity. In the case of the $r$ band, we estimate this systematic error to be $\sigma_{\mathrm{zpt}} \simeq 0.005$. This calibration error is different for each filter, with the upper limit being set by the filters in the blue-end of the spectrum. These show a calibration error of approximately 0.04 magnitudes. These calibration uncertainties are consistent with the ones cited in Sect. 3.5. We stress, however, that the current statistics is too low to properly use this procedure to estimate calibration errors. This will not be a problem for J-PAS, where the area observed will be three orders of magnitude larger than miniJPAS, and the J-PAS strategy includes a larger fraction of overlapping areas.

\subsection{Comparison with other surveys}

We further test the quality of the data by comparing our photometry with that from other surveys. The broad band photometry is compared with that from SDSS and HSC-SSP, while the narrow band photometry is compared with synthetic photometry derived by convolving SDSS spectra with the J-PAS filters, as detailed below:
Broad band photometry. We compare the broad band photometry of miniJPAS with that of SDSS and HSC-SSP. We use sources with no warnings or flags and the MAG_AUTO photometry, which is a proxy for the total magnitude. For this comparison we use colors, as they best reflect the SEDs of the sources. Colours are also very useful to check the quality of the relative calibration in miniJPAS. We show in Fig. 14 the $(r-i)$ color difference between miniJPAS and SDSS/HSC-SSP as a function of the miniJPAS $r$-band for both point-like and extended sources (point sources are defined as having CLASS_STAR $>0.9$ ). The colors in miniJPAS are generally consistent with those in SDSS and HSC-SSP. The lack of systematic shifts in the comparison with SDSS confirms the quality of the calibration. As expected, the scatter is larger in the comparison with SDSS than with HSC-SSP, because of the shallower depth reached by SDSS. We obtain similar results when comparing the colors from other broad bands. The good agreement for both point and extended sources confirms not only the quality of the calibration, but also that we can trust the SEDs of galaxies for extragalactic studies.

Narrow band photometry. We compare the narrow-band photometry of miniJPAS with the synthetic photometry obtained by convolving SDSS spectra with the transmission curves of the J-PAS filters. We use the spectra available in the SDSS DR12 of galaxies having magnitudes in the miniJPAS $r$-band $r \leq 20$ and $20 \leq r \leq 22.5$ in the MAG_PSFCOR photometry. We used the MAG_PSFCOR photometry to avoid aperture corrections. In any case, we scaled the spectra (in AB magnitudes) to the median of the $g, r, i$ miniJPAS MAG_PSFCOR photometry to account for the differences in aperture between the SDSS fibre and the miniJPAS photometry. In total, there are 405 spectra at $r \leq 22.5$, of which 122 at $r \leq 20$. Figure 15 shows the mean of the difference between the observed and the synthetic magnitudes for each narrow band filter. The mean of the shifts is $0.006 \pm 0.028$ and the median scatter is 0.06 . These small values confirm the accuracy of the miniJPAS narrow-band calibration. The scatter is larger for galaxies with $20 \leq r \leq 22.5$ than for brighter objects due to the shallower depth reached by SDSS, and it is larger in the blue than the red narrow-band filters.

\section{3. miniJPAS completeness}

The limiting fluxes at which a survey is not biased in object detection, also known as the completeness of a survey, is one of the key parameters that must be determined during the preliminary analysis and data validation phase. The J-PAS survey will include a pipeline for determining the completeness curves of the composite images or tiles. Here, we first introduce our methodology and the completeness curves obtained for the miniJPAS survey, and then compare these with the results obtained from a comparison with HSC-SSP.

Our pipeline is based on the use of synthetic images of galaxies and stars, which are treated separately. The completeness of each pointing is obtained after injecting at random positions without previous detections a position-dependent PSF for pointlike sources, and galaxy light profiles extracted from COSMOS HST images in F814W for extended sources. In the latter case, an average color term is applied to modify the structure measured in F814W to the appropriate scale in each of the miniJPAS filters, and the original profile is convolved with the corresponding position-dependent PSF. The completeness curves are then calculated from the fraction of injected sources that our source detection process recovers (see Sect. 3.4). This analysis is done for each of the miniJPAS pointings separately in 


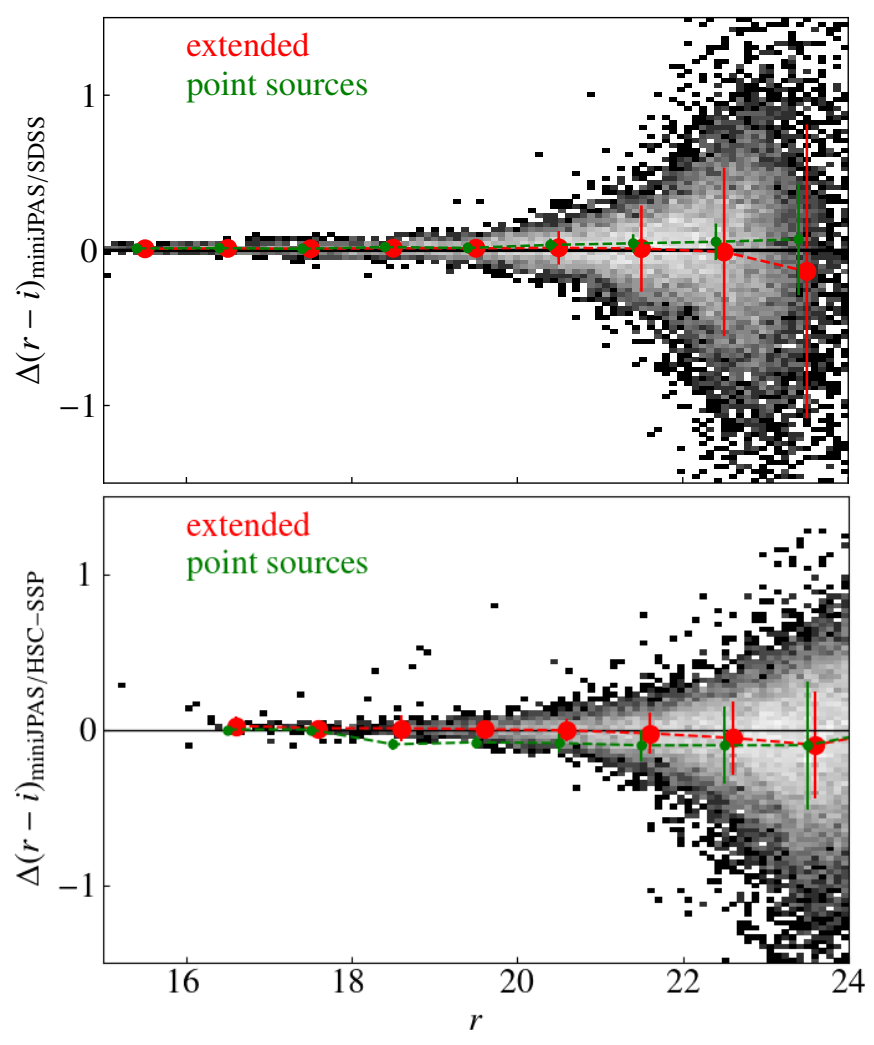

Fig. 14. Color $(r-i)$ difference of miniJPAS and SDSS (upper panel) and HSC-SSP (lower panel) as a function of the miniJPAS $r$ band. The gray density map is for all objects. The colored points indicate the median with $1 \sigma$ error in different magnitude bins for extended (red) and point sources (green).

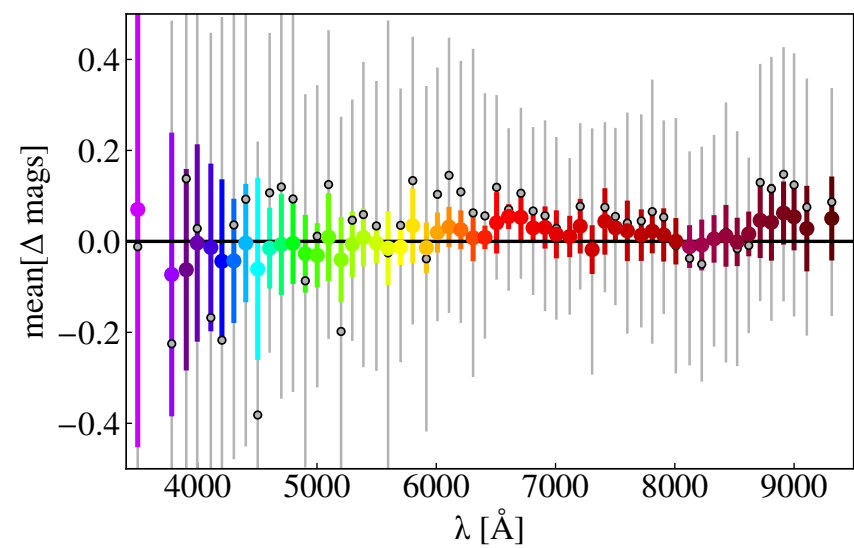

Fig. 15. Difference between miniJPAS photometry (MAG_PSFCOR magnitudes) and synthetic photometry derived by convolving SDSS spectra with the J-PAS filters. Coloured symbols, and thick error bars, correspond to galaxies with $r<20$, while gray circles with thin error bars to galaxies with $20<r<22.5$. The error bars refer to the scatter of the distribution within each filter.

order to account for possible discrepancies between the pointings related to, for example, the different observational conditions. In Fig. 16 we show the derived completeness curves for the compact and extended sources. The curves are properly fitted by a Fermi-Dirac distribution function (see e.g. Sandage et al. 1979; Díaz-García et al. 2019a) of the form:

$C=\frac{100}{\exp \left\{\left(r_{\mathrm{JPAS}}-C_{50}\right) / \Delta_{C}\right\}+1}$,

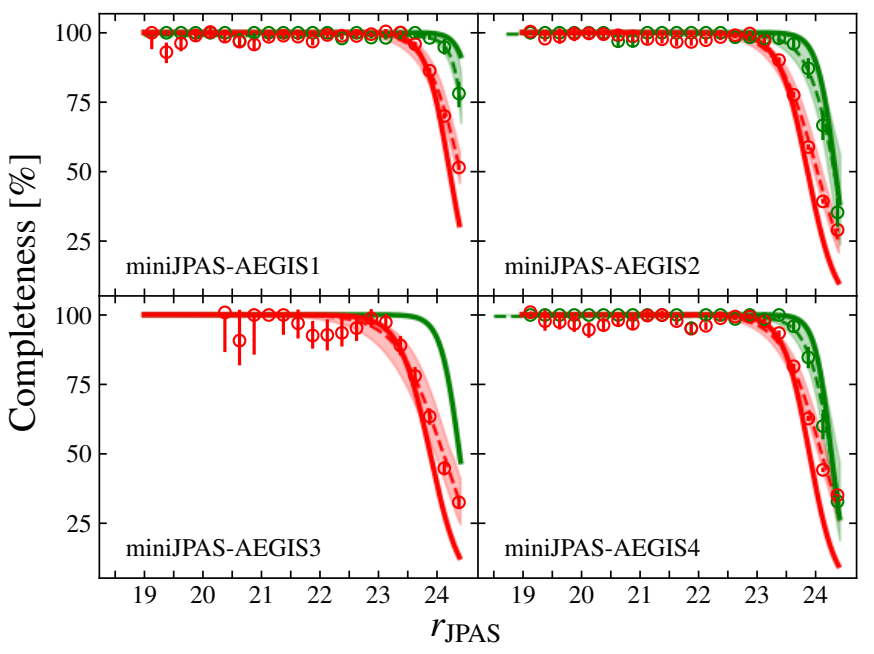

Fig. 16. Completeness of extended and point-like miniJPAS sources as a function of total magnitude in the miniJPAS $r$ band for the four miniJPAS pointings. The solid lines are the completeness curves of extended and point-like sources (red and green, respectively) obtained from the injection and detection of synthetic sources. The red (green) circles illustrate the corrected fraction of common extended (pointlike) sources in the miniJPAS and HSC-SSP surveys (this comparison is not possible for miniJPAS-AEGIS3, given the small area of overlap between this pointing and HSC-SSP). The dashed colored lines are the completeness curves obtained from the comparison with the HSC-SSP dataset, with uncertainties highlighted by the shaded area.

where $C$ is the detection fraction (in per cent units), $C_{50}$ is the magnitude at which the sample is $50 \%$ complete, and $\Delta_{C}$ the decay rate on the fraction of detections. In Table 10, we show the parameters that result from fitting Eq. (1) to the miniJPAS data. We estimate that the sample of point-like sources in the full miniJPAS catalog is $99 \%$ complete up to $r \sim 23.6 \mathrm{mag}$ (MAG_AUT0). For extended sources, this limit is constrained to be $r \sim 22.7 \mathrm{mag}$. It is worth mentioning that the miniJPAS-AEGIS1 field is deeper than the other pointings, with limiting depths of $r \sim 24.1$ and $r \sim 23.3 \mathrm{mag}$ for point-like and extended sources, respectively.

With a typical seeing of $0.6-0.8$ arcsec in the optical range, along with a depth of $r \sim 26$ mag ( $5 \sigma$ limit within a 2 arcsec diameter aperture), the HSC-SSP survey can also be used to test the miniJPAS completeness. To carry out the comparison, we used the second data release of the HSC-SSP survey (Aihara et al. 2019) as the reference catalog for the HSC-SSP photometry. The overlap area with miniJPAS is $0.7 \mathrm{deg}^{2}$. Taking as reference the $r$ band from HSC-SSP (cmodel magnitudes, which are a proxy for total magnitudes), we computed the fraction of detections in common between HSC-SSP and miniJPAS as a function of magnitude following a similar procedure as the one described above for the injection of synthetic sources. Owing to the lower seeing of the HSC-SSP observations, we used as compactness discriminator the parameter r_extendedness_value provided by the HSC-SSP catalogs. To perform a robust and fair determination of the completeness, we quadratically include the systematic uncertainties reported by Huang et al. (2018) for both PSF and cmodel magnitudes (see Tables 1 and 3 in Huang et al. 2018). The impact of these uncertainties on the fraction of common detections is determined via a Monte Carlo approach by assuming Gaussian errors for the $r_{\text {HSC }}$ fluxes. Nevertheless, discrepancies between the HSC-SSP and the miniJPAS observations (e.g., seeing and deblending) may yield systematic discrepancies in the fraction of common 
Table 10. Parameters describing the completeness function of the miniJPAS survey obtained from injection and recovery of synthetic sources $\left(C_{50}\right.$ and $\Delta_{C}$, see Eq. (1)), and from measuring the fraction of sources in common between miniJPAS and the much deeper HSC-SSP catalogs $\left(C_{50}^{\text {HSC }}\right.$ and $\left.\Delta_{C}^{\mathrm{HSC}}\right)$.

\begin{tabular}{lccccc}
\hline \hline Field & $C_{50}$ & $\Delta_{C}$ & $C_{50}^{\mathrm{HSC}}$ & $\Delta_{C}^{\mathrm{HSC}}$ & $r_{99}^{\text {AUTO }}$ \\
\hline Point-like & & & & & \\
\hline miniJPAS-AEGIS1 & 24.74 & 0.14 & $24.57_{-0.09}^{+0.09}$ & $0.13_{-0.06}^{+0.06}$ & 24.10 \\
miniJPAS-AEGIS2 & 24.33 & 0.16 & $24.33_{-0.12}^{+0.16}$ & $0.23_{-0.08}^{+0.11}$ & 23.59 \\
miniJPAS-AEGIS3 & 24.39 & 0.16 & - & - & 23.65 \\
miniJPAS-AEGIS4 & 24.26 & 0.14 & $24.26_{-0.10}^{+0.12}$ & $0.20_{-0.07}^{+0.08}$ & 23.62 \\
\hline Extended & & & & & \\
\hline miniJPAS-AEGIS1 & 24.22 & 0.21 & $24.39_{-0.08}^{+0.07}$ & $0.29_{-0.06}^{+0.09}$ & 23.26 \\
miniJPAS-AEGIS2 & 23.86 & 0.24 & $24.03_{-0.07}^{+0.07}$ & $0.33_{-0.03}^{+0.04}$ & 22.76 \\
miniJPAS-AEGIS3 & 23.88 & 0.26 & $24.11_{-0.15}^{+0.14}$ & $0.39_{-0.07}^{+0.09}$ & 22.69 \\
miniJPAS-AEGIS4 & 23.89 & 0.22 & $24.09_{-0.07}^{+0.07}$ & $0.34_{-0.04}^{+0.04}$ & 22.88 \\
\hline
\end{tabular}

Notes. In the last column, we give the limiting values for a completeness level of 99\% for the miniJPAS detection band ( $r$, MAG_AUT0). We find that total magnitudes, used for the injection of synthetic sources, are essentially equivalent to AUT0 magnitudes at the $99 \%$ completeness limit.

sources, typically ranging from 5 to $10 \%$, even when both surveys are not biased by incompleteness.

To account for this effect and obtain alternative completeness curves based on HSC-SSP data, we corrected the fraction of common detections, assuming that these discrepancies are linearly dependent on the $r_{\mathrm{HSC}}$ magnitude. For the point-like sources, this correction of the fraction of common detections proved unnecessary and it was only performed for the extended sources. Figure 16 illustrates the fraction of common detected sources in the HSC-SSP and the miniJPAS surveys for extended and point-like sources, as well as the fits following Eq. (1) after correcting for discrepancies between the HSC-SSP and the miniJPAS observations. We find a good agreement between the completeness curves obtained from the synthetic sources and those from the comparison with HSC-SSP observations (within the $1 \sigma$ uncertainty level, see also Table 10$)$. We were not able to observationally determine the completeness curve of point-like sources in the miniJPAS-AEGIS3 pointing because of the small area in common with HSC-SSP.

We note that the completeness curves were obtained from total (synthetic) magnitudes in miniJPAS and from cmodel magnitudes in HSC-SSP. Regarding the practical application of the completeness curves, the AUTO magnitude is our best proxy for the total magnitude and should thus be used to define a complete flux-limited sample. Therefore, the galaxy size adopted to derive the completeness for extended objects is the KRON_RADIUS (i.e., the aperture used to compute the AUTO magnitude of each source, see Sect. 4.1). The limiting magnitudes found for all the miniJPAS pointings for the dual mode catalogs are $r_{\text {AUTO }}=23.6$ and 22.7 mag for point-like and extended sources, respectively. The completeness curve parameters are available in the ADQL table minijpas. TileImage.

\subsection{Purity}

As a complement to completeness, purity indicates the fraction of spurious sources for different magnitude cuts. We estimate the purity of the miniJPAS catalog using the negative-image technique and, as for the completeness, we compare the results with the purity estimated using the HSC-SSP deeper photometric catalogs. In the negative image technique, the first step is to remove from the original images the objects detected by SExtractor. The resulting images are then multiplied by -1 , which generates "negative images". SExtractor is then run again on these "negative images" and, by construction, any detection is spurious. Assuming a symmetric background noise distribution around zero, the amount of spurious sources detected in this way is similar to that of the original images ${ }^{33}$. Due to small differences in the computed background RMS of the "negative" images with respect to the original ones, mainly due to the removal of the objects, the DETECT_THRESH must be tuned to ensure that the detection parameters are the same as those used on the original images. This procedure was applied to the co-added $r$ images and the purity estimated as:

$P=\frac{N_{\text {obj,cat }}-N_{\text {obj,neg }}}{N_{\text {obj,cat }}}$,

where $N_{\text {obj,cat }}$ is the number of sources detected by SExtractor in the original images, included in the miniJPAS catalogs, and $N_{\text {obj,neg }}$ is the number of sources detected in the "negative" images. In Fig. 17 we show the purity fraction as a function of magnitude calculated with this approach. Full purity is achieved up to $r \sim 24$ in all the miniJPAS pointings. At fainter magnitudes, purity starts dropping quickly, as expected. In the same figure, we also show the purity fraction calculated from the comparison with HSC-SSP data. In this case, the contamination by spurious detections is calculated from the number of sources in the miniJPAS catalogs (excluding the spurious ones as calculated above) that do not have a counterpart in HSC-SSP, in the area where the two surveys overlap and taking into account the masks of the two surveys. The results are also shown in Fig. 17 for the three pointings where HSC-SSP data is available. While predicting full purity down to fainter magnitudes, it is reassuring that both approaches agree in the regime where we expect to carry out most J-PAS science.

\footnotetext{
33 This procedure takes into account only pure statistical spurious detections and not those produced by other causes such as, for example, spikes around bright stars. However, we consider that at the faintest magnitudes the spurious detections due to statistical fluctuations of the background dominates the population.
} 


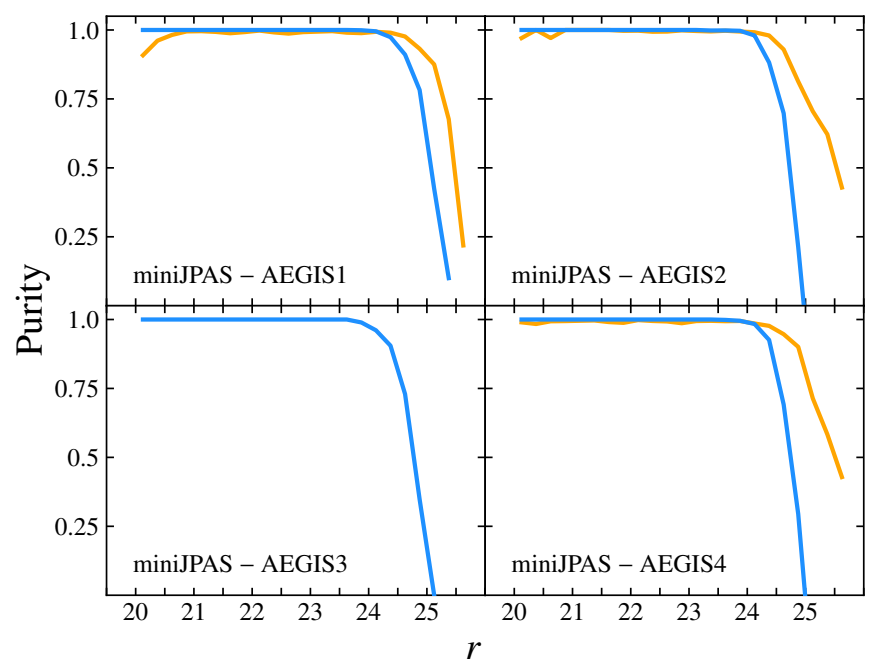

Fig. 17. Purity of the miniJPAS catalog, calculated with the "negative images" technique (blue curves) and from the cross-match with the deeper HSC-SSP data (orange curves). The cross-match with HSC-SSP is not possible for miniJPAS-AEGIS3 because of the small overlapping area.

\subsection{Number counts}

We present the stellar and galaxy number counts in Fig. 18. To derive the number counts separately for point-like and extended sources, we used the stellar-galaxy locus classification presented in Sect. 4.2.1, corrected for the completeness derived from the synthetic source injection as described above.

The number counts derived from the Bayesian classification agree with the expectations from the literature both for stars and galaxies up to $r=23.5 \mathrm{mag}$, as shown in Fig. 18. The derived probabilities are publicly available in the ADQL table minijpas. StarGalClass.

\subsection{Discussion of data quality issues and lessons learned}

Here, we discuss the known issues and caveats related to the miniJPAS data-release presented in this paper and reflect on the main lessons learned in view of the upcoming J-PAS survey.

\subsubsection{Caveats and known problems}

We provide below a brief list of issues and caveats to keep in mind when using the data-release presented in this paper:

Inhomogeneous exposure times. Narrow bands present heterogeneous total exposure times from band to band and from pointing to pointing as, in some cases, more than the planned number of images was used to produce the co-adds. For J-PAS, the observational strategy with the JPCam will be defined to ensure that the depths are more homogeneous across the survey area and the different filters.

Extended faint sources. The superbackground subtraction described in Appendix B, together with the small dithering pattern of miniJPAS, causes extended sources to be potentially confused with the instrumental background. Therefore, for miniJPAS data we cannot discard the possibility that some faint, extended real sources have been totally or partially removed from the final images. For J-PAS data, this issue is not expected because of the relatively large dithering length of one half of the CCD.

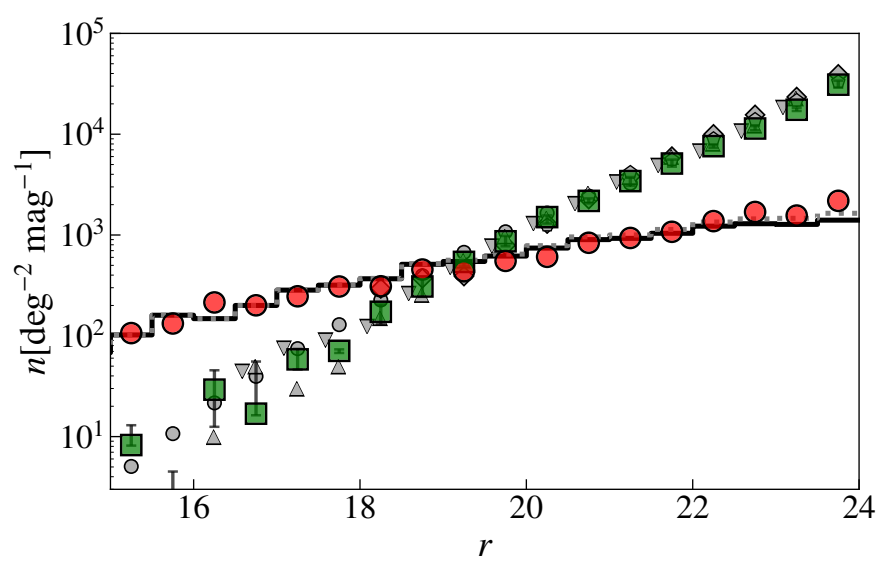

Fig. 18. Stellar (red dots) and galaxy (green squares) number counts as a function of the $r$ band magnitude. The black solid curve shows the stellar number counts at the EGS position estimated with the default TRILEGAL model of the Milky Way (Girardi et al. 2005). The dotted gray curve includes the expected contribution for point-like quasars (from the BOSS survey, Palanque-Delabrouille et al. 2016) to the compact population. Gray symbols are galaxy number counts from the literature: Yasuda et al. (2001, circles); Huang et al. (2001, triangles); Kümmel \& Wagner (2001, inverted triangles); Kashikawa et al. (2004, diamonds); and Capak et al. (2004, pentagons).

Image quality. Since the time window to complete the miniJPAS project was limited, the initial requirements in terms of seeing, airmass, and atmospheric stability were at times relaxed to guarantee the completion of the whole project on time. This has resulted in PSFs that were on average larger than what the system can theoretically provide ( $<1$ arcsec). Also, some thin clouds were present during observations of the last tray with the reddest filters (mainly J0790 and redder), which was detected only after the data reduction.

Fringing correction. The limited time available for the miniJPAS observations resulted in a number of scientific images which turned out to be too scarce for generating high-quality, master fringing images for all red filters. This meant that the final fringing removal was not optimal in all cases. This is not expected to be a problem for J-PAS, as a very large number of exposures will be available in each band, allowing for the master fringing patterns to be determined with higher accuracy.

Astrometric accuracy of the coadded images. Despite single individual images having astrometric solution accuracies with respect to Gaia DR2 of the order of $\sim 0.03 \operatorname{arcsec}^{34}$, these degrade to up to $\sim 0.3$ arcsec in the case of coadded images and dual-mode catalogs. Resampling and pixel rotations before coadding seem to be the main source of this effect. This will be revisited in future data releases.

\subsubsection{From miniJPAS to J-PAS: Lessons learned}

The miniJPAS survey presented in this paper has provided us with a crucial benchmark test before commencing the much larger J-PAS. The acquisition, reduction, and storage of miniJPAS data have taught us important lessons on the handling of such complex data, which is proving to be extremely valuable in the preparation for J-PAS. We note that the data processing

\footnotetext{
${ }^{34}$ Measured as the rms of the differences between Gaia DR2 coordinates and the final astrometric solution for the stars used in the astrometric calibration.
} 
of miniJPAS relied heavily on the infrastructure and methods developed for J-PLUS. The pipeline developed for J-PLUS, from data acquisition to publication, needed only few changes to process the miniJPAS data, allowing us to focus on the unexpected issues. Below we list some of the key lessons on topics ranging from the data acquisition to the data publication relevant to the upcoming J-PAS:

Data acquisition. The JPAS-PF camera has allowed us to advance with the commissioning of the T250 early, saving precious time after the installation of JPCam. Despite the intrinsic differences between JPCam and JPAS-PF, we also gathered useful information on the operations, such as the determination of the right observational conditions for the acquisition of sky flatfield images.

Data transfer. The data transfer from the OAJ to the UPAD, described in Cristóbal-Hornillos et al. (2014), proceeded as expected, and we do not foresee any issue nor any need for modifying the upcoming J-PAS data.

Data processing. During the data processing phase, we had to face the first issues related to the actual characteristics of our system (telescope, camera, and filters). Since there are important differences between JPAS-PF and JPCam, we focused primarily on those issues related to the telescope (e.g., reflections) or to the filters. In other words, we tried to avoid applying too many resources to solving problems that are unique to JPAS-PF that will not be present in JPCam. Vignetting and background patterns, however, are two issues that we do not expect to be severe for JPCam, but we considered these important enough for the miniJPAS survey to properly address them, as discussed in Sect. 3.1. For these issues, we tried to develop solutions that could be adapted to JPCam, if necessary. Vignetting causes a reduction of the effective useful area of the CCD. Theoretically, this effect should be corrected with a flat-field correction. However, this assumes a perfect stability of the shadowing pattern, which is not the case, even for JPAS-PF which was located at the center of the focal plane. For miniJPAS data we opted for the more conservative and straightforward solution: trimming all the images to ensure that vignetting did not affect the final survey area. However, for a large project such as J-PAS, this extreme solution would impact strongly the speed of the survey. Therefore, we are developing for J-PAS a more sophisticated solution which selects the proper area for each individual exposure. Regarding the background patterns, miniJPAS data has shown how these depend on several aspects related with the telescope, camera, and filters, and how this dependency can change in time scales of minutes. Part of the problem was linked to the illumination issues. This is treated with the illumination correction and a solution valid also for JPCam images was implemented ${ }^{35}$. For the remaining background patterns we implemented the super-background subtraction. We developed a general solution, valid for the different kinds of patterns found in miniJPAS and assuming that J-PAS may show similar, although not equivalent, behaviour. The use of all the J-PAS filters for miniJPAS has been very useful, as it allowed us to understand issues related to specific wavelengths. This was particularly true for the case of fringing, where the reddest filters are the ones most affected.

Data publication. The steps related to data publications were tested during the DR1 of J-PLUS. Only few changes were

\footnotetext{
${ }^{35}$ In fact, this effect is also present in J-PLUS data and, therefore, it was implemented to the data processing of the DR2 of J-PLUS, showing the advantages of having instruments with similar properties.
}

needed to adapt the system to miniJPAS and we do not foresee the need for any further modifications for the publication of J-PAS data.

All the aspects described here underline the importance of having a test-bench data set to train and prepare the full pipeline. The experience based on J-PLUS has been of paramount importance for a relatively smooth processing of miniJPAS. In turn, miniJPAS has allowed us to acquire a deeper knowledge of the system to fully prepare for the arrival of J-PAS.

\section{A glimpse at J-PAS science with miniJPAS}

In this section, we use the miniJPAS data to give a brief overview of the wide variety of scientific cases that can be addressed thanks to the J-PAS filter system. In Fig. 19 we show examples of astrophysical objects observed in miniJPAS. The measured fluxes in the narrow filters are shown and compared with the SDSS spectra. The top six panels show examples of J-spectra for stellar sources, where the classes and spectral types listed for each object were retrieved from the SDSS spectroscopic pipeline database. Figure 19 shows that the miniJPAS photometry is capable of successfully capturing the continuum shape of these stellar sources as well as the main spectral absorption features used to determine spectral types, atmospheric parameters, and chemical abundances (e.g., carbon, $\alpha$-elements). For hotter stars (first four panels), the hydrogen Balmer lines are clearly traceable at $\lambda<5000 \AA$, while for cooler stars (middle and right panels in the second row) several TiO molecular bands can be distinguished at $\lambda>6000 \AA$.

The J-spectra are sufficiently detailed to allow for the derivation of stellar parameters and to address a range of important topics in stellar astrophysics, including the search for metalpoor stars (Youakim et al. 2017; Yoon et al. 2018; Placco et al. 2018, 2019), blue horizontal branch stars (Santucci et al. 2015a; Whitten et al. 2019a; Starkenburg et al. 2019), blue stragglers (Santucci et al. 2015b), ultracool dwarfs, and white dwarfs. They also allow for the identification and chemical characterization of Galactic stellar streams, classical dwarf galaxies, and ultra-faint dwarf galaxies (e.g., Longeard et al. 2018, 2020; Shipp et al. 2018, 2019; Chiti et al. 2020). Systems such as planetary nebulae and symbiotic stars, which involve circumstellar ionized gas, are also easily identified and characterized based on the J-spectra, (Gutiérrez-Soto et al. 2020). The J-PAS photometry can also contribute to the study of stellar populations of Globular Clusters as shown by Bonatto et al. (2019) for the GC M15, using J-PLUS Science Verification Data.

Examples of J-spectra of extragalactic sources observed by miniJPAS are provided in the bottom six panels of Fig. 19. Ordered by increasing redshift, the first four panels show examples of both passive and star forming galaxies. Clearly visible are the $4000 \AA$ break of red galaxies and the $\mathrm{H} \alpha$ emission line of blue star-forming objects. The last two panels show examples of quasars, with the profiles of their broad emission lines, such as Ly $\alpha, \mathrm{CIV}, \mathrm{CIII}]$, clearly traced by our filters. As these examples clearly show, the J-PAS filter system allows for the detection and characterization of emission lines with equivalent widths larger than a few $\AA$ (Martínez-Solaeche et al. 2021) in star forming galaxies, AGN, and quasars. $\mathrm{H} \alpha$ and [OII] $\lambda \lambda 3726,3729$ can be used to trace the star forming population from the local universe up to $z \sim 0.5$ and $z \sim 1.5$, respectively. Weaker lines, such as $\mathrm{H} \beta$ and $[\mathrm{OIII}] \lambda 5007$, can also be easily detected for galaxies with $r<20$ and $z<0.35$, the same magnitude and redshift range probed by the GAMA survey (Driver et al. 2011). This shows 

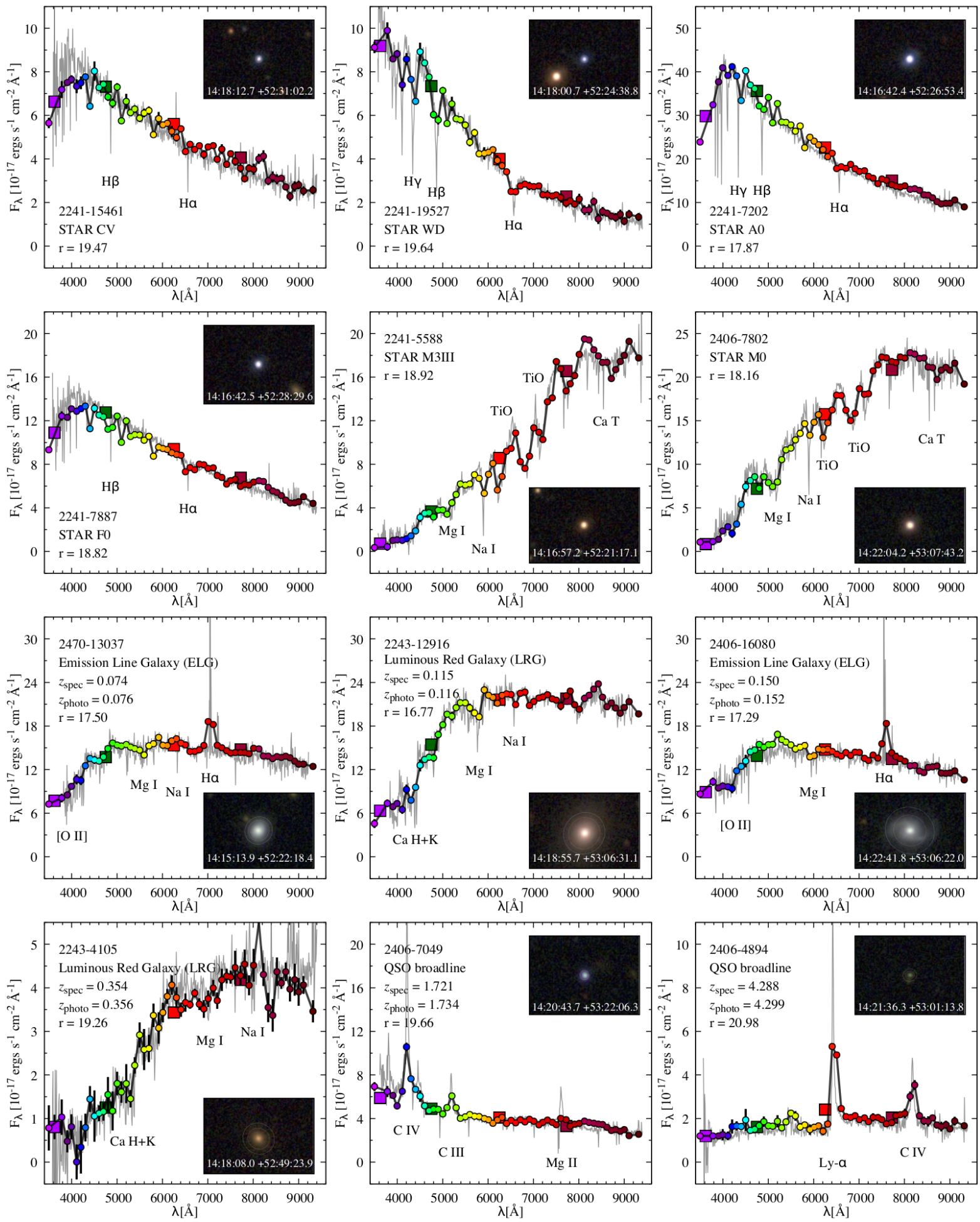

Fig. 19. J-spectra of different classes of stars, galaxies, and quasars in the miniJPAS field (colored symbols) compared with the SDSS spectra (gray lines). The miniJPAS object ID, $r$ magnitude, and classification are listed in the legend. Extragalactic sources are ordered by increasing redshift, and both spectroscopic (SDSS) and photometric (miniJPAS) redshift are provided in the legend. The multi-color inset images are centred on the object and are 30 arcsec across. The ellipses visible for extended sources are the 〈auto ellipse $\rangle$ (inner ellipses) and the 〈petro ellipse $\rangle$ (outer ellipses).

that miniJPAS (and, in the future, J-PAS) can be used to identify star forming galaxies without the redshift limitations of typical narrow-band surveys, and any of the pre-selection biases typical of spectroscopic surveys. Below, we use the miniJPAS data to provide concrete examples of future scientific applications of J-PAS data.

\subsection{J-PAS: a low spectral resolution IFU survey}

The combination of the wide FoV, large survey area and relatively small CCD pixel size will make J-PAS a competitive IFU-like survey of extended galaxies at $z<0.15$, where the low spectral resolution will be compensated by the large number 

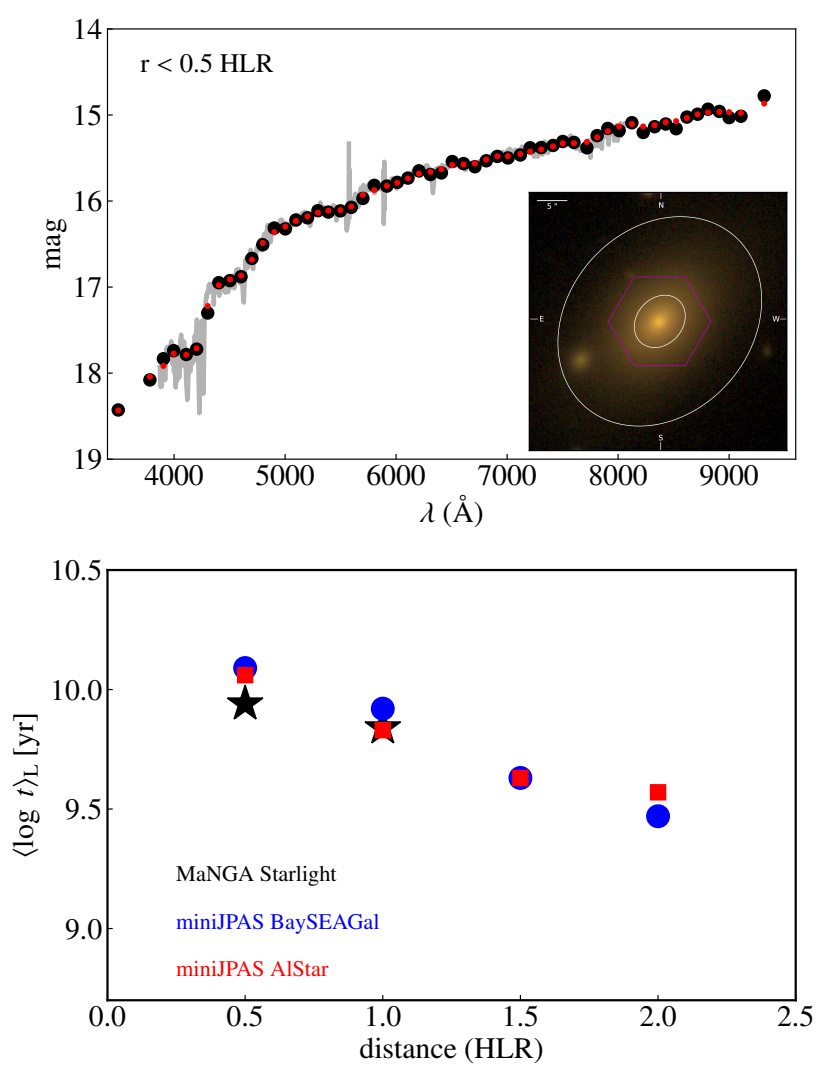

Fig. 20. Spatially-resolved properties of a galaxy at $z \sim 0.1$. Upper panel: MaNGA spectrum (grey line) of the central annulus, up to 0.5 HLR, compared with miniJPAS data (black dots). The result of the fitting of miniJPAS data is also plotted (red dots). The inset shows an image of the galaxy in the $r$ band where two ellipses are overlaid at 0.5 and 2 HLR. The FoV of the MaNGA survey is over-plotted as a red hexagon. Bottom panel: comparison of the radial variation of the average age $\langle\log t\rangle_{L}$ derived from miniJPAS data, with the non-parametric code AlStar (red dots) and the parametric code BaySEAGal (blue dots), and from the MaNGA data analysed with the STARLIGHT code (black stars).

statistics that the survey will provide. In this sense, J-PAS will be highly complementary to CALIFA (Sánchez et al. 2012, 2016; García-Benito et al. 2015) and MaNGA (Bundy et al. 2015; Law et al. 2015) by providing much larger samples of galaxies and by probing larger galactocentric distances, up to several halflight-radii (HLR) ${ }^{36}$, with a significantly better spatial sampling ( 0.23 arsec pixel ${ }^{-1}$ for the broad bands and 0.46 arsec pixel $^{-1}$ for the narrow bands). For example, even at $z \sim 0.1$, where the spatial scale is $\sim 1.8 \mathrm{kpc} \mathrm{arcsec}^{-1}$, galaxies with a typical HLR of $4 \mathrm{kpc}$ can be studied every $\sim 0.5 \mathrm{HLR}$, assuming an average seeing of 1 arcsec. Moreover, J-PAS data will not suffer from FoV restrictions, so the spatial properties of the largest galaxies in the Local Volume (distance $\leq 15 \mathrm{Mpc}$ ) can be studied in a uniform manner.

In the small miniJPAS footprint there are a number of extended galaxies with a HLR exceeding 5 arcsec that are useful for showing the potential of J-PAS. We have chosen, as an example, a quiescent galaxy at $z \sim 0.1$ of $14.7 \mathrm{mag}$ in the $r$ band. This galaxy ( $\alpha=14: 15: 20.37 ; \delta=52: 20: 45.19)$ was also observed by the MaNGA survey (Bundy et al. 2015), so

\footnotetext{
${ }^{36} \mathrm{We}$ define the half-light-radius as the semi-major axis length of the elliptical aperture which contains half of the light of the galaxy at the rest frame wavelength $5600 \AA$.
}

we can directly show how results based on the analysis of the miniJPAS data compare with those from the spectroscopic data. The MaNGA data were retrieved from the SDSS DR14 (Abolfathi et al. 2018), and the spectra were analysed using the PyCASSO pipeline (de Amorim et al. 2017). From the miniJPAS data we define the peack of the galaxy emission at $5600 \AA$, and we estimate the HLR to be 9.4 arcsec.

The top panel of Fig. 20 shows a comparison between the MANGA spectra and the miniJPAS photometric data within 0.5 HLR, demonstrating the very good agreement in terms of flux and SED. We derive the spatially-resolved stellar population properties of the galaxy by analysing both the miniJPAS and MaNGA data in annuli centred on the nucleus. While the MaNGA FoV allows us to study the galaxy properties in the first two annuli (within 1 HLR, see the inset in Fig. 20), with the miniJPAS data we can extend the analysis up to 2 HLR. The miniJPAS J-spectra of the four annuli were fitted using the BaySEAGal software (de Amorim et al., in prep.), a Bayesian code that allows a wide variety of intrinsic galaxy properties to be derived, such as the stellar mass, the age of the stellar population, the metallicity, and internal extinction. For comparison, we also fit the J-spectra with the non-parametric code AlStar (Cid Fernandes et al., in prep.).

Both codes use the same set of single stellar populations (SSP) models from Bruzual \& Charlot (in prep.) to derive the star formation histories. The magnitudes obtained from the fitting with BaySEAGal are plotted in Fig. 20. The MaNGA spectra of the two inner annuli are instead fitted with the non-parametric code STARLIGHT (Cid Fernandes et al. 2005; López Fernández et al. 2016), following the same processes used for CALIFA data (de Amorim et al. 2017) and using the same set of SSPs. We note that AlStar is a spin-off of BaySEAGal and was specifically developed to deal with photometric data. Thus, any differences between the results from these two codes are purely due to differences between the spectroscopic and photometric data. We find very good agreement in the stellar population properties derived from the parametric and non-parametric codes (AlStar and BaySEAGal) for the miniJPAS data, and these results also agree with those derived from the MaNGA spectroscopic data. In the bottom panel of Fig. 20 we show the derived radial distribution of luminosity-weighted stellar ages $\langle\log t\rangle_{\mathrm{L}}$. A similar negative age gradient was also found in the CALIFA sample (González Delgado et al. 2015), and in a few extended galaxies in the ALHAMBRA survey (San Roman et al. 2018). The stellar mass in the inner 0.5 HLR is estimated to be $10^{11.10} M_{\odot}$ and $10^{11.06} M_{\odot}$ from the MaNGA and miniJPAS analysis, respectively.

J-PAS will also be competitive for IFU-like studies of star forming galaxies of the nearby Universe. The HII regions distributions and their ionized gas properties will be retrieved for galaxies in the Local Volume $(z<0.015)$, in similar way as it has been done for J-PLUS (Logroño-García et al. 2019, following the methodology of Vilella-Rojo et al. 2015). Several methodologies can be developed to retrieve the emission line flux for galaxies out of the Local Volume. Martínez-Solaeche et al. (2021) presented a Neural Network method that can be used to determine the equivalent width of $\mathrm{H} \alpha, \mathrm{H} \beta$, [NII] $\lambda 6584$, [OIII] $\lambda 5007$ using spectra from the CALIFA and MaNGA surveys as a training set.

\subsection{Morphological studies in multi-color}

The morphologies of galaxies, derived from the analysis of photometric data, can vary with wavelength, as different stellar 

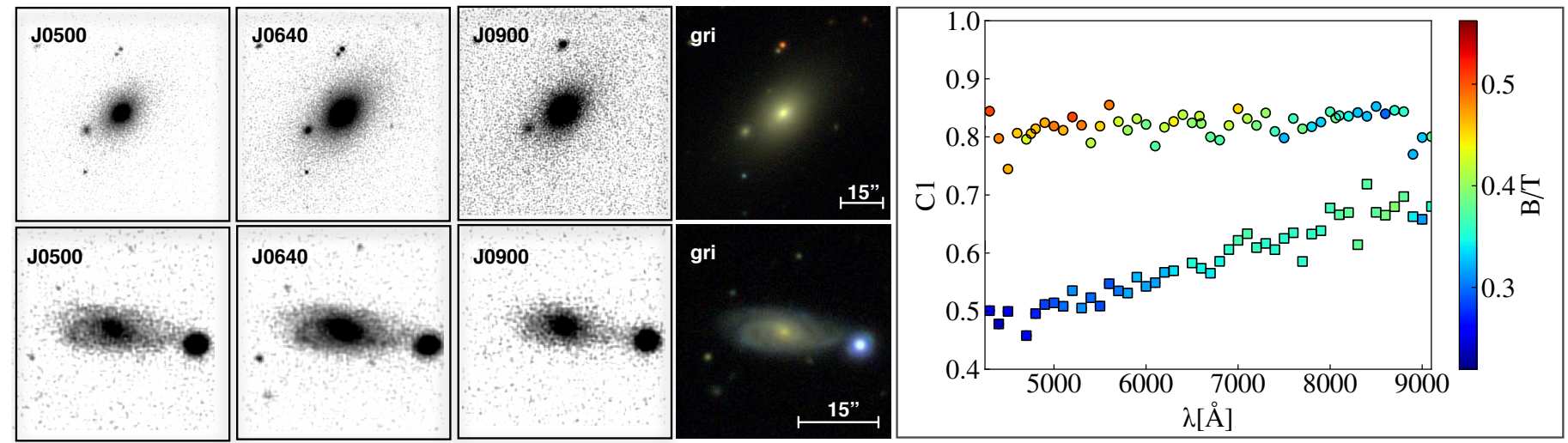

Fig. 21. Galaxy morphology across wavelengths. Left panel: miniJPAS images of an elliptical galaxy, ID 2470-10239 (upper row) and a spiral galaxy, ID 2470-10291 (bottom row), in three of the J-PAS narrow bands (J0500, J0640, J0900), as an example of the variation of galaxies' properties with wavelength. Middle panel: $g, r, i$ color images of the two galaxies. The bar of 15 arcsec in length, indicated on the images for reference, corresponds to $21.5 \mathrm{kpc}$ for the elliptical galaxy $(z \simeq 0.075)$ and $20.8 \mathrm{kpc}$ for the spiral galaxy $(z \simeq 0.073)$. Right panel: variation of the concentration $\mathrm{C} 1$ parameter with wavelength, color-coded according to the bulge-to-total light ratio $(B / T)$, for the early type galaxy (circles) and the spiral galaxy (squares). The magnitudes are integrated up to 1 disk scale length.

populations present different colors. For example, if the disk of a galaxy is characterized by a young, star-forming population, while its spheroidal component is old and metal rich, the Sérsic index will increase toward redder wavelengths (Vulcani et al. 2014; Vika et al. 2015). Several works have explored the variation of morphological parameters with wavelengths, recovering the star formation history and stellar population of the different galaxy components. The typical number of fitted wavelengths has been increasing during the years, from, for instance, the five broad bands of the SDSS survey (ugriz, Bottrell et al. 2019) to the nine of the GAMA survey (SDSS + UKIDSS, Kennedy et al. 2016) and the $\simeq 15$ bands in the Dustpedia project (GALEX, SDSS, 2MASS/UKIDSS, WISE, Davies et al. 2017). J-PAS data will be unique for this type of studies, as it will provide a lowresolution spectrum for each galaxy component and CAS (concentration, asymmetry, smoothness) parameters in each of filters. Vika et al. (2013) showed, using SDSS data, that a multi-band bulge-to-disk light decomposition is reliable up to $z \sim 0.3$, while the CAS parameters can be used to define morphological properties up to $z \sim 0.25$ using SDSS data (Ferrari et al. 2015) and even up to $z \sim 3-4$ using Hubble Space Telescope data (Conselice 2003; Overzier et al. 2010).

Figure 21, generated using miniJPAS data, gives a taste of the potential of J-PAS. The first three panels show how an early- and a late-type galaxy appear at different wavelengths. While the isophotal intensity of the early type galaxy increases with increasing wavelength, since the majority of its mass comprises old and metal rich stars, for the late type galaxy the spiral arms, where most star formation occurs, fade away at the redder wavelengths. The colors of the different components are also clearly visible in the middle $g, r, i$ color image. More quantitatively, we show in the right panel, for both galaxies, the variation with wavelength of the concentration $C 1^{37}$, which ranges from 0 to 1 . This was derived using the non-parametric code MFMTK (Ferrari et al. 2015). The symbols are color-coded according to the bulge-to-total light ratio, obtained performing a bulge-to-disk light decomposition of the galaxy images using GALFITM, a multi-wavelength extension of GALFIT (Peng et al. 2011). For the early type galaxy the concentration is nearly

\footnotetext{
37 The concentration $C 1$, first of the three CAS parameters, is defined as follows: $C 1=\log \left(R_{80} / R_{20}\right)$, where $R_{80}$ and $R_{20}$ are the radii containing, respectively, $80 \%$ and $20 \%$ of the total light inside the Petrosian Region.
}

constant with wavelength, while for the late type galaxy $C 1$ increases with increasing wavelength. The spiral galaxy is diskdominated $(B / T<0.25)$, while in the early type galaxy the bulge and the disk contribute almost equally to the galaxy light. Interestingly, while for the spiral galaxy the $B / T$ ratio increases with increasing wavelength, (i.e., the percentage of light associated with the bulge is higher in redder bands), in the early-type galaxy the bulge is slightly more prominent in bluer bands. Such techniques as the Kurvature method of Lucatelli \& Ferrari (2019) allow for the structural multi-components of a galaxy to be to disentangled to give an indication of the transition regions and allow for the presence of other features to be inferred, such as bars and nuclear disks. These kind of measurements, together with the SED fitting of the J-spectra, will allow us to obtain a full picture of the build-up of galaxy structures (Cortesi et al., in prep.).

In conclusion, the J-PAS filter system will provide accurate galaxy morphologies and structural and stellar population properties over the entire optical range. The large area of J-PAS will furthermore allow for searches of peculiar and rare objects in all kinds of environments, such as ram-pressure stripped (including "jellyfish") galaxies (e.g., Roman-Oliveira et al. 2019; Wang et al. 2020) and green peas and Lyman Break Analogs (e.g., Heckman et al. 2005; Cardamone et al. 2009; Loaiza-Agudelo et al. 2020).

\subsection{Galaxy properties across cosmic time}

The accuracy of the photometric redshifts and the physical properties of galaxies derived from the J-spectra, will allow us to characterize the galaxy population from the nearby universe up to $z \sim 1$. By fitting the continuum, we can constrain stellar population properties of galaxies, as well as segregate galaxy populations according to their star-formation activity (see, e.g., Cid Fernandes et al. 2005; Mathis et al. 2006; Walcher et al. 2011; Moustakas et al. 2013). The ability of the J-PAS narrow band-filters in extracting physical parameters of galaxies has been studied in Mejía-Narváez et al. (2017) for a variety of star formation histories. The authors used SED fitting codes (TGASPEX and DynBaS Magris et al. 2015) to investigate biases, correlations, and degeneracies affecting the retrieved parameters in mock galaxies. They also compared 


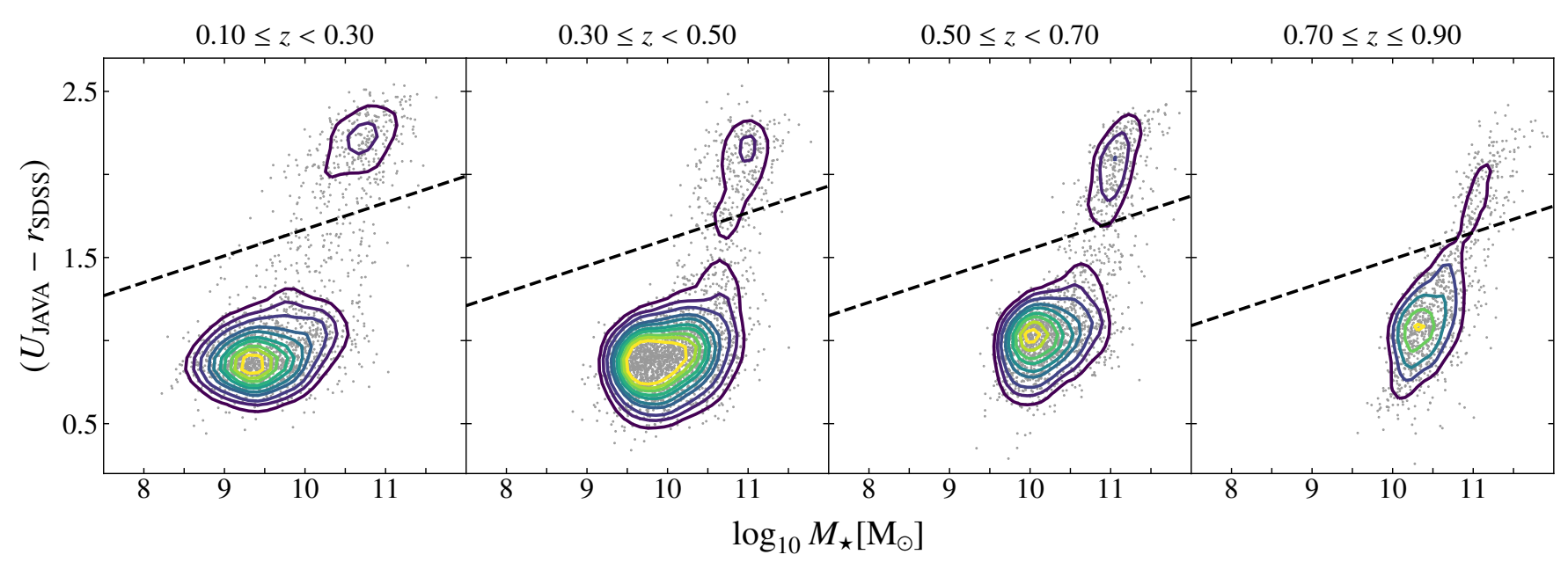

Fig. 22. Rest-frame stellar mass-color diagram corrected for extinction, showing the distribution of star-forming (lower cloud) and quiescent (upper cloud) galaxies at four redshift intervals. The dashed black line illustrates the lower color limit of quiescent galaxies as a function of stellar mass and redshift. Yellow (purple) contours illustrate higher (lower) densities.

results obtained from SDSS galaxy spectra to narrow-band SEDs (synthesised from the same spectra), concluding that the J-PAS filter system yields the same trend in the age-metallicity relation as spectroscopy for typical $\mathrm{S} / \mathrm{N}$ values. With the data introduced in Sec. 6.1, González Delgado et al. (2021) study a sample of galaxies with several SED fitting codes (MUFFIT by Díaz-García et al. 2015; TGASPEX by Magris et al. 2015; AlStar by Cid Fernandes et al., in prep.; BaySEAGal by de Amorim et al., in prep.), additionally showing that J-PAS photometry yields the same trend in the age-mass, color (rest and intrinsic)-mass as the analysis of spectroscopic data.

In the example of Fig. 22, we used the MUlti-Filter FITting code (MUFFIT, Díaz-García et al. 2015) to separate star-forming and quiescent galaxies via a rest-frame stellar mass-color diagram corrected for extinction (Díaz-García et al. 2019a). In this specific analysis, we used the single stellar population models of Bruzual \& Charlot (2003) to build twoburst composite stellar population models, assuming the extinction law of Fitzpatrick (1999). The accurate determination of the intrinsic extinction of galaxies, which needs precise and well-calibrated photospectra, is essential to differentiate quiescent galaxies from dusty star-forming galaxies and it is also important for the selection of green valley galaxies to avoid contamination from obscured star-forming galaxies (Brammer et al. 2009; Cardamone et al. 2010; Moresco et al. 2013; Díaz-García et al. 2019a). Other interesting properties related to the stellar contents of galaxies and its evolution (such as formation epoch and age, environment effect, quenching mechanisms, metallicity, initial stellar mass function) can be studied in a self-consistent way (González Delgado et al. 2021), making it possible to carry out a proper analysis and interpretation of the correlations between different physical parameters (see Belli et al. 2015; Martín-Navarro et al. 2015; Díaz-García et al. 2019b,c, and references therein).

Quasars and AGN can also be easily identified and characterized from their J-spectra (Abramo et al. 2012; Chaves-Montero et al. 2017). We used miniJPAS data to test a new method that estimates the photo- $z$ of quasars by means of a PCA modelling of the spectral variations (Queiroz et al., in prep.). The eigenspectra of the PCA are computed using as basis a selection of SDSS spectra of broad-line quasars (Yip et al. 2004, see also Abramo et al. 2012) and they correspond to the most relevant modes of variation of broad-line quasars. In addition to these eigenmodes, we also include a reddening law to better fit reddened quasar spectra. Our method employs an optimization routine tuned to extract the full redshift probability distribution. The quality of the derived photometric redshifts for quasars at $z_{\text {spec }}<3.5$ with $r<22$ is estimated using a subsample of 97 quasars in the miniJPAS footprint with $\operatorname{med}(\mathrm{S} / \mathrm{N})^{38} \geq 5$, reliable spectral identification in SDSS from Pâris et al. (2018) (mask flag and zWarning flag equal to zero), and for which a visual inspection did not show any calibration issues within the miniJPAS observations. The result is shown in Fig. 23. The estimated average photo- $z$ uncertainty is 0.0059 and the fraction of outliers is $4.1 \%$. We notice that photo- $z$ uncertainty decreases for $z>2$ thanks to the fact that multiple strong emission lines are present within the J-PAS spectral coverage at those high- $z$. With the larger samples provided by J-PAS, we expect to be able to further fine-tune our methods and reach even higher redshift precision to determine quasar luminosity functions and the search for rare quasars at high redshifts.

At $z>2$ we are also sensitive to bright Ly- $\alpha$ emitters (LAEs). The wide spectral coverage of the J-spectra will allow us to distinguish those from quasars (e.g., Spinoso et al. 2020), making J-PAS highly complementary to surveys studying LAEs with a few narrow bands (e.g., Ouchi et al. 2018) or with spectroscopic instruments with limited spectral coverage, such as HETDEX (Hill et al. 2008). In the next section we will discuss the power of J-PAS in performing clustering studies using the large samples of emission line galaxies at $z<1$ and quasars and LAEs at $z>2$ expected.

\subsection{Large scale structure}

As already discussed and demonstrated in previous sections, J-PAS was designed as a high-completeness astrophysical survey (Benítez et al. 2009, 2014), with a set of narrow-band filters able to provide photospectra with sufficient spectral resolution to yield excellent photo- $z$ (see Sect. 4.2.2).

The ability of J-PAS to map the cosmic web is illustrated in Fig. 24, where we show a slice of the volume mapped by the spectroscopic DEEP2 and by the photometric miniJPAS counterparts. Across a wide redshift range, miniJPAS is

${ }^{38}$ Where the $\operatorname{med}(\mathrm{S} / \mathrm{N})$ is defined as the median value of the ratio flux/flux_err for all filters with detection. 


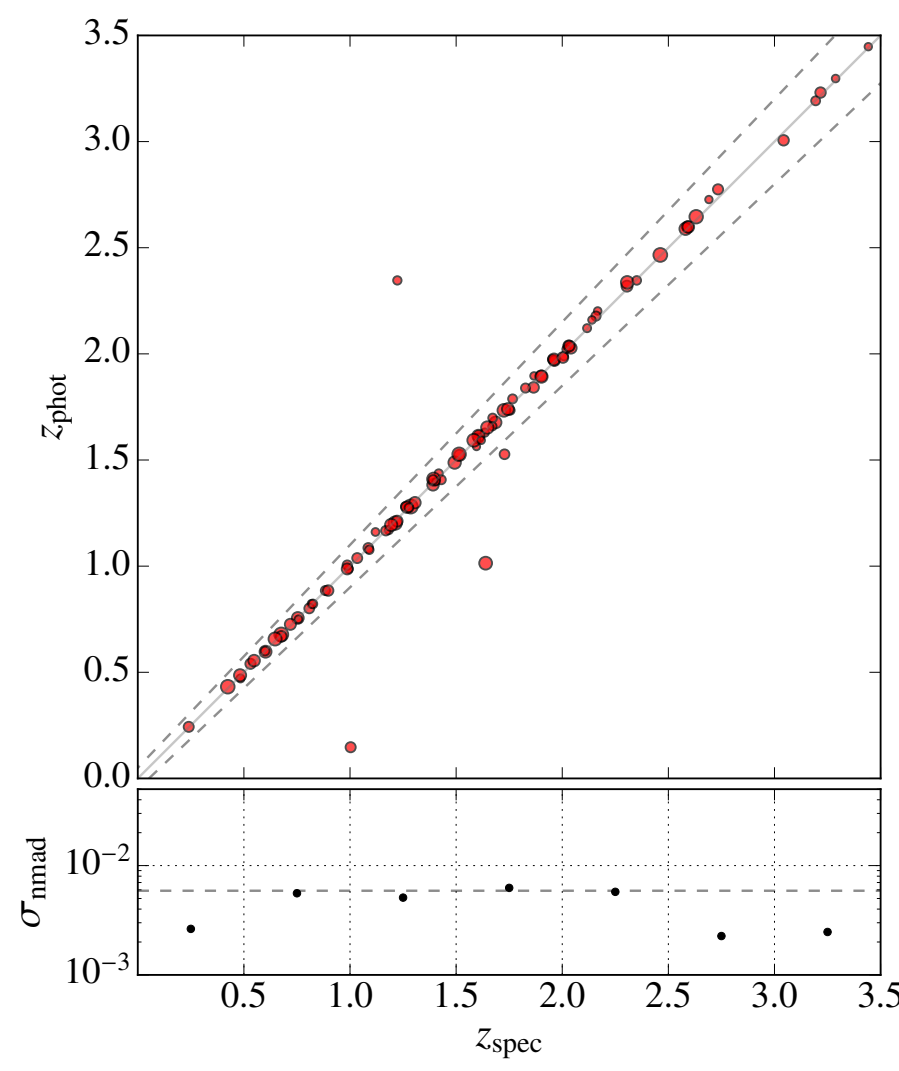

Fig. 23. Photo- $z$ of miniJPAS quasars. Upper panel: photometric versus spectroscopic redshifts for a sample of 97 DR14 quasars with $z_{\text {spec }}<3.5$ detected in the miniJPAS field. Larger symbols represent higher $\operatorname{med}(\mathrm{S} / \mathrm{N})$. The solid diagonal line indicates $z_{\text {phot }}=z_{\text {spec }}$, and the dashed lines correspond to $z_{\text {phot }}=z_{\text {spec }} \pm 0.05\left(1+z_{\text {spec }}\right)$. The average photo- $z$ uncertainty, $\sigma_{\text {nmad }}$, is 0.0059 and the fraction of outliers is $4.1 \%$. Bottom panel: photo- $z$ precision as a function of redshift, with the horizontal dashed gray line indicating the average photo- $z$ uncertainty.

able to reproduce the pattern of spatial clustering of galaxies unveiled by the spectroscopic DEEP2 survey. The impact of the line-of-sight blurring due to the photo- $z$ errors becomes visible in particular for objects with low values of photo- $z$ accuracy (ODDS). This typically affects scales along the line-of-sight below $\lesssim 50 h^{-1} \mathrm{Mpc}$. Therefore, J-PAS will be able to accurately map the cosmic structures, from the largest to mildly non-linear scales, where most of the cosmological information is encoded. In particular, it will be possible to constrain cosmological models using the filamentary structure of the universe (Tempel et al. 2014; Kitaura et al. 2021).

Due to its large area, high density of objects, and accurate photo- $z$, J-PAS is expected to impact the field of cosmology in several different ways. First, since the entire sample of galaxies has photo- $z$ errors lower than $0.8 \%$ (see Sect. 4.2.2), we will have the ability to to extract the scale of baryonic acoustic oscillations (BAO) from galaxy clustering up to $z \sim 1$, both in the transverse and in the radial directions (see, e.g., Chaves-Montero et al. 2018, for the impact of photometric redshifts on clustering measurements). This analysis will produce accurate measurements of the angular-diameter distance $D_{\mathrm{a}}(z)$ and the Hubble parameter $H(z)$ (Blake \& Glazebrook 2003; Seo \& Eisenstein 2003), allowing us to tightly constrain the dark energy equation of state and the other cosmological parameters. Second, being an imaging survey which does not need to rely on the pre-selection of targets, we will be able to measure the shape of the power spectrum (or, equivalently, the corre- lation function) using many different tracers of the large-scale structure (Seljak 2009; McDonald \& Seljak 2009; Abramo et al. 2016): galaxies of different spectral types, colors, luminosities and stellar masses (Arnalte-Mur et al. 2014; Hurtado-Gil et al. 2016; Montero-Dorta et al. 2020), quasars (Abramo et al. 2012), Ly- $\alpha$ emitters (Gurung-López et al. 2019, 2020), or even groups and clusters. The multi-tracer character of J-PAS will lead to improved constraints not only on dark energy but also on modified gravity models, primordial non-Gaussianities, and neutrino masses (Abramo \& Bertacca 2017). In particular, J-PAS has the potential to provide the most precise determination of the Hubble parameter $H(z)$ and the growth rate $f \sigma_{8}(z)$ at $0.2 \leq z \leq 0.6$, outperforming past and upcoming surveys in this redshift range. A dedicated forecast analysis can be found in Costa et al. (2019), Resco et al. (2020), and Resco \& Maroto (2021).

Another indicator of the power of J-PAS to constrain cosmology is the signal-to-noise ratio $(\mathrm{S} / \mathrm{N})$ which we can use to measure galaxy clustering. For a galaxy with bias $b_{\mathrm{g}}$, the amplitude of its clustering in Fourier space is given by $P_{\mathrm{g}}=b_{\mathrm{g}}^{2} P_{\mathrm{m}}(k)$, where $P_{\mathrm{m}}(k)$ is the matter power spectrum. The main source of noise is shot (count) noise, $1 / \bar{n}_{\mathrm{g}}$, where $\bar{n}_{\mathrm{g}}$ is the comoving galaxy number density. Since the matter power spectrum at the typical BAO scales $\left(k_{\mathrm{BAO}}=0.1 \mathrm{~h} \mathrm{Mpc}^{-1}\right)$ is on the order of $P_{\mathrm{m}}\left(k_{\mathrm{BAO}}\right) \sim(1+z)^{-2} \times 10^{4} h^{-3} \mathrm{Mpc}^{3}$ and galaxy bias is on the order of $b_{\mathrm{g}} \sim 1-2$, so that we may rise above the level of shot noise; thus, a galaxy survey should achieve number densities of $\bar{n}_{\mathrm{g}} \gtrsim(1+z)^{2} \times 10^{-4} h^{3} \mathrm{Mpc}^{-3}$. In Fig. 25 we show the estimated (comoving) spatial number density of galaxies at different redshifts for different color and ODDS cuts, based on the photo- $z$ results of miniJPAS (see Sect. 4.2.2) for extended sources with $r<22.5$. These estimates account for the masked regions in the miniJPAS footprint, and incorporate the statistical weights correcting for the stellar contamination (López-Sanjuan et al. 2019b). As expected, we find that blue/late-type galaxies are significantly more abundant than red/early-type ones. Moreover, the photo- $z$ precision for blue galaxies depends significantly on redshift, while that for red galaxies is approximately constant. Typically, we find number densities in the range of $\left[10^{-3}, 5 \times\right.$ $\left.10^{-2}\right] h^{3} \mathrm{Mpc}^{-3}$, significantly higher than past spectroscopic surveys such as LOWZ+CMASS and comparable to the photometric pencil-beam surveys such as COSMOS or ALHAMBRA and the DESI spectroscopic survey. We can select galaxy samples of progressively higher photo- $z$ quality by applying cuts in the ODDS parameter (see details in Sect. 4.2.2), at the cost of reducing the sample completeness. In Fig. 25 we show that for a cut in ODDS of 0.6 , we still obtain fairly large number densities, namely, in the range of $\left[10^{-4}, 10^{-2}\right] h^{3} \mathrm{Mpc}^{-3}$. The redshift precision associated with each ODDS cut depends on redshift, as shown in Fig. 26, where the complicated relation between redshift precision and expected number density of sources is shown for different ODDS cuts and redshift slices. An ODDS cut of 0.6, as used in Fig. 25, generally ensures a relative redshift error of 0.004 or lower. The previous results, together with the completeness shown in Fig. 16, suggest that J-PAS will be able to perform very well in the redshift range of $0.2<z<0.6$ (Resco et al. 2020; Costa et al. 2019).

In addition to measuring clustering on large scales, J-PAS will also study galaxy correlations on very small scales (a few tens of kiloparsecs, sampling the so-called 1-halo term) due to the high number densities of detected sources. In particular, since J-PAS is not affected by fiber-collision problems in the way spectroscopic surveys are, the small scale limit is set only by the combination of seeing and photometric redshift accuracy. Beyond standard clustering analyses, the J-PAS data will enable 


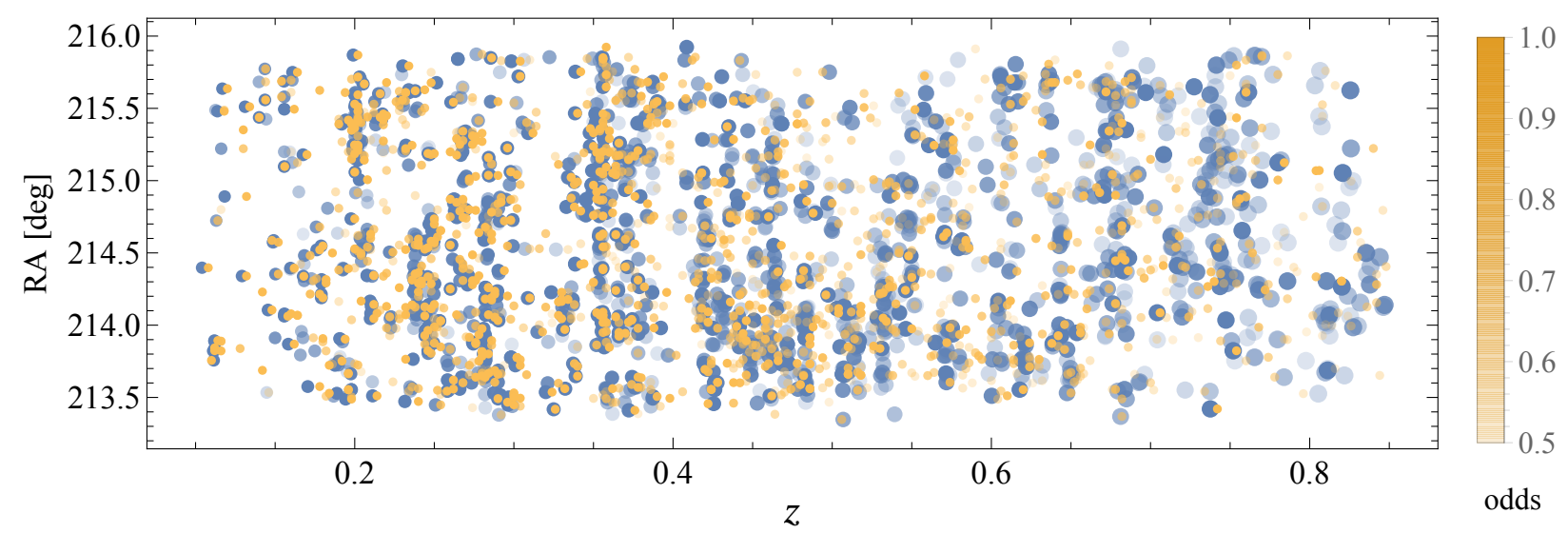

Fig. 24. Slice of miniJPAS as mapped by the spectroscopic sample of DEEP2 (blue symbols) and the miniJPAS counterparts (orange symbols), for $r<22.5$. miniJPAS successfully reproduces the pattern of spatial clustering of galaxies unveiled by the spectroscopic DEEP2 survey. The redshifts of miniJPAS sources are given by the best photo- $z$ solution, as described in Sect. 4.2.2. The color shade of the miniJPAS/DEEP2 galaxy pairs is related to the ODDS value of the miniJPAS photo- $z$ solution. The size of the symbols of DEEP2 sources is given by the target photo- $z$ precision, $\sigma_{\mathrm{NMAD}}=0.003 \times(1+z)$, which is reached when the constant-size miniJPAS symbol lies within the DEEP2 one.

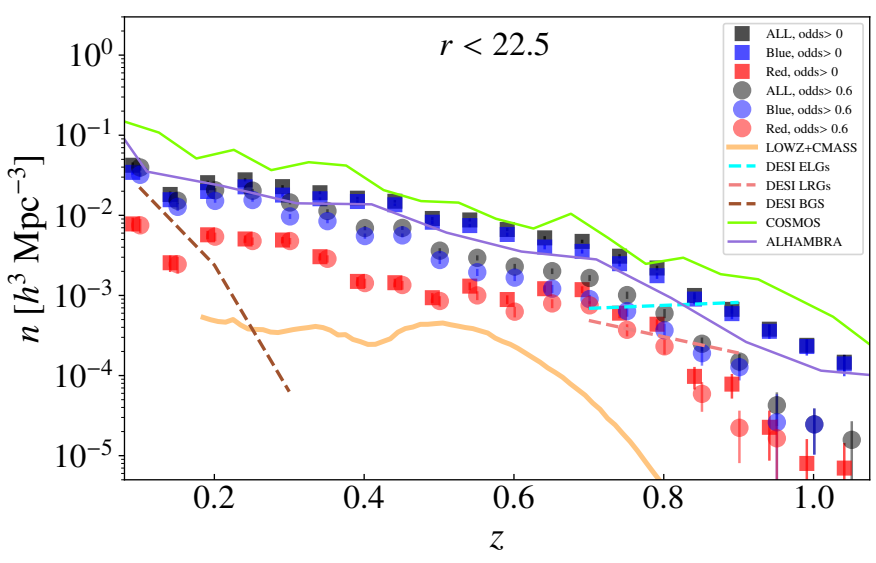

Fig. 25. Comoving number density of galaxies in miniJPAS, as a function of redshift. The total galaxy population (dark gray squares) is broken up into star-forming (blue squares) and quiescent (red squares) galaxies. We also apply a cut in photo- $z$ precision (ODDS $>0.6)$ (circles, with the same color coding as the squares). We show the number density of galaxies for other surveys such as LOWZ+CMASS (orange line), DESI (DESI Collaboration 2016, brown, cyan, and magenta solid lines, respectively, for BCGs, ELGs, and LRGs), ALHAMBRA (Molino et al. 2014, purple line, with an applied cut of F644W < 22.5, where F644W is a medium-band centred at $6440 \AA$ ) and COSMOS (Laigle et al. 2016, green line, with an applied cut of $r<22.5$ ).

a series of alternative cosmological tests. For instance, the redshift precision of J-PAS will allow us to analyse angular redshift fluctuations (ARF, Hernández-Monteagudo et al. 2021a) in narrow redshift shells $(\Delta z \sim 0.01)$. This new cosmological observable contains information about the bias and peculiar velocities of the probes, and thus is sensitive to gravity and nonGaussianity parameters from inflation, among others. A tomographic analysis (such as that of Hernández-Monteagudo et al. 2021b) would hence provide a detailed view of the redshift evolution of source bias, peculiar velocity amplitudes, and a number of other cosmological quantities that can be derived thereof. The combination of ARF with standard 2D angular clustering maps can be cross-correlated with current and future $\mathrm{CMB} / \mathrm{sub}$-millimeter maps as well as other 2D maps derived

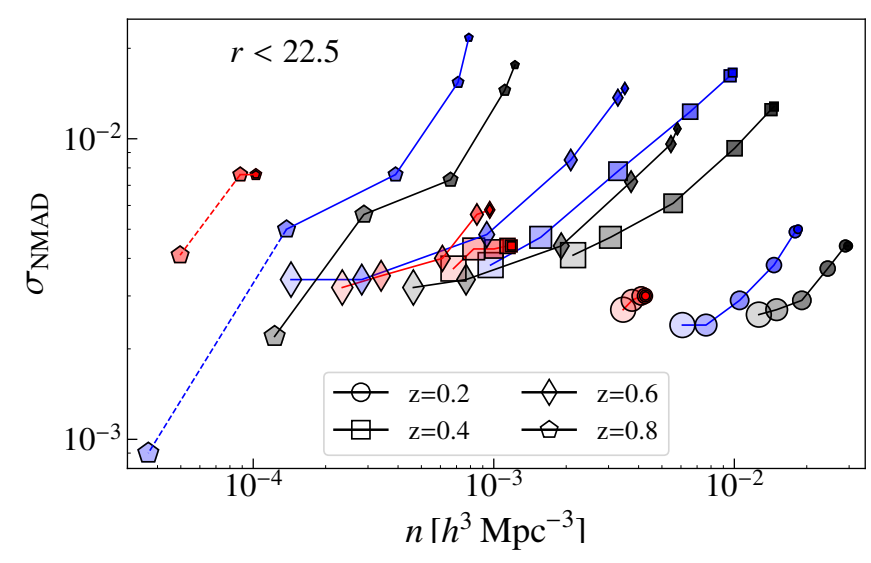

Fig. 26. Relation between galaxy number density and corresponding redshift error, as a function of redshift, ODDS cut, and galaxy color. Red galaxies, blue galaxies and the full sample are shown in red, blue, and black lines and symbols, respectively. Results are presented in redshift bins of $\Delta z=0.2$, centred at $z=0.2$ (circles), $z=0.4$ (squares), $z=0.6$ (diamonds) and $z=0.8$ (pentagons). For each redshift, the symbols correspond to the cumulative number density for increasing cuts, at ODDS > 0.0, 0.2, 0.4, 0.6, 0.8, 0.9 (for some cuts the symbols are overlapping). The larger the size of the symbols, the larger the ODDS cut (thus the redshift precision). Dashed lines correspond to the regime where we have, in the miniJPAS field, less than 15 galaxies.

from them, such as the CMB lensing convergence map, maps of the cosmic infrared background, or maps of the thermal Sunyaev-Zel'dovich effect (Sunyaev \& Zeldovich 1972). Given its high number density of galaxies (reaching $>10^{-2} \mathrm{~h}^{3} \mathrm{Mpc}^{-3}$ at $z<0.5)$, J-PAS will also be able to map the cosmic web in rich detail, revealing the pattern of sheets, filaments, and voids over an unprecedented volume, and providing a powerful test of the cosmological model as well as of our theory of structure formation (Kitaura et al. 2021). Another potential field where J-PAS may contribute significantly is the study of emission from unresolved sources in different (and narrow) redshift shells: the narrow bands should allow for the isolation of line emitters placed at particular redshifts, even when these lack the required $\mathrm{S} / \mathrm{N}$ to be identified as sources in the 
catalog. Crucial for this intensity mapping (IM) approach (see, e.g., Gong et al. 2011) are the adopted techniques for background removal, which may impact IM signal on the largest scales. Finally, J-PAS will have the unique ability to measure the cosmic shear (see Sect. 6.6) in the same volume in which the galaxy power spectrum is measured, thus probing different combinations of the gravitational potentials with two independent observables.

At the highest redshifts $(z>2)$, cosmological studies will be possible via quasars and Ly- $\alpha$ emitters (LAEs). Because of their strong emission features, these objects can be easily detected and characterized with the J-spectra. Using miniJPAS, we tested the quasar photo- $z$ uncertainty for objects at $r<22$, being on average $\sim 0.5 \%$, but decreasing to $0.3 \%$ for objects at $z>2$ (see Sect. 6.3). Even at fainter magnitudes (up to $r \sim 23$ ) we expect to recover complete quasar samples (with a density of $\sim 200 \mathrm{deg}^{-2}$ ) with $\sim 1 \%$ redshift precision, allowing for efficient spectroscopic follow-up campaigns. The WEAVEQSO survey (Pieri et al. 2016) will target $\sim 400 k$ J-PAS quasars at $z>2$, which will allow for precise Ly- $\alpha$ forest and IGM studies. Finally, at $z>2$, J-PAS will select large samples of LAEs to perform cosmological studies. This will be highly complementary to the HETDEX spectroscopic survey (Hill et al. 2008), as the lower redshift precision of J-PAS will be compensated by the larger area and wider spectral coverage.

\subsection{Galaxy clusters}

Galaxy clusters are key to discriminate among the various cosmological models as they can be used to place constraints on $\Omega_{\mathrm{m}}$ and $\Omega_{\Lambda}, w$ and $\sigma_{8}$ (e.g., see Allen et al. 2011, for a review). Ascaso et al. (2016) applied the Bayesian Cluster Finder (Ascaso et al. 2012) and calculated the selection function for J-PAS from $N$-body+analytical mock catalogs similar to the ones of Merson et al. (2013) adapted to J-PAS. Thanks to the exquisite photo- $z$ that J-PAS can deliver, they found the minimum halo mass threshold for detection of galaxy systems with both $>80 \%$ completeness and purity to be $M_{\text {halo }} \sim 5 \times 10^{13} M_{\odot}$ up to $z \sim 0.7$, outperforming DES (DES Collaboration 2020), ACTpol (Hilton et al. 2018) and SPTpol (Bleem et al. 2020), and comparable to eROSITA (Merloni et al. 2012) and LSST (LSST Science Collaboration 2009) at lower-intermediate redshifts. This is a unique feature of J-PAS, that will allow for the expansion of the study of the evolution of galaxy systems down to galaxy groups. These low-mass systems are particularly interesting and poorly observed at higher redshifts and J-PAS will allow us to clarify many of their unusual properties, such as the relative importance of different mechanisms that can explain their low baryon fraction (e.g., Dai et al. 2010), for example by comparing stellar loss to intracluster light (Jiménez-Teja et al. 2018, 2019) to IGM ejection (e.g., Bower et al. 2008) due to the energetic excess by central AGN. Moreover, galaxy star formation activity has been suggested to vary over an extended range of environments, measured as a function of galaxy density, and redshift (e.g., Elbaz et al. 2007; Paulino-Afonso et al. 2020). Therefore, the statistical study of galaxy properties in groups will allow us to probe the evolution of galaxies in intermediate environments.

To demonstrate the strength of our multi-band data for the selection of groups and clusters (which we refer to only as "clusters" hereafter), we searched for clusters in miniJPAS within the redshift range of $0.05<z<0.8$ with the Voronoi Tessellation (VT) technique (Ramella et al. 2001; Lopes et al. 2004), the Adaptive Matched identifier of Clustered Objects

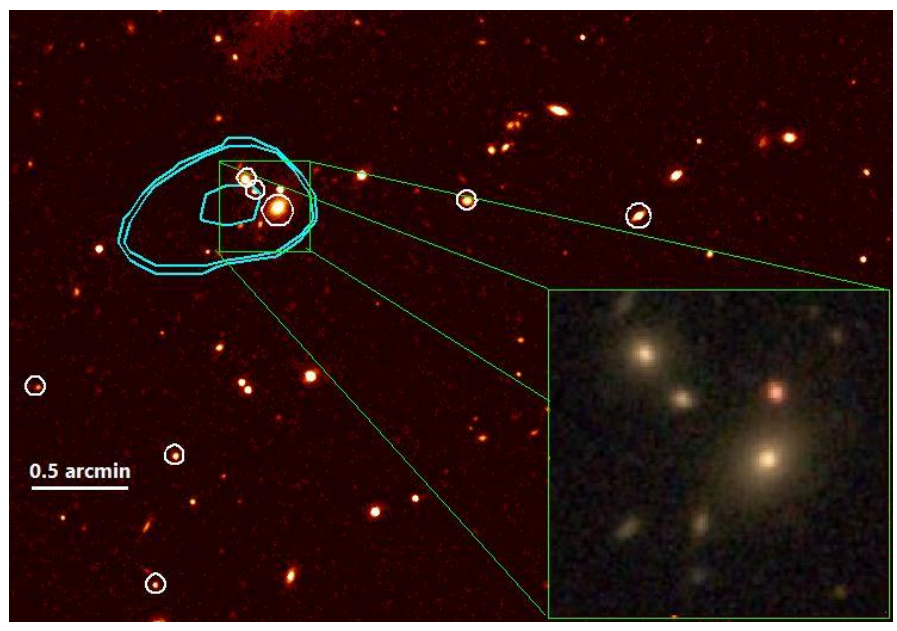

Fig. 27. Example of a low-mass cluster (group), mJPC 2241-16656, detected by all three cluster finder algorithms in the miniJPAS/Chandra AEGIS field. The system, dominated by the galaxy SDSS J141719.86+523537.6 is at $\mathrm{RA}=14: 17: 20 \mathrm{Dec}=+52: 35: 38$ and has photo- $z=0.238$. Overlaid on the $i$-band image, white circles mark the position of galaxy members (within a window of $\pm 4 \sigma_{\text {l.o.s. }}$ - or $\left.840 \mathrm{~km} \mathrm{~s}^{-1}\right)$ within $R_{200}\left(507 h_{70}^{-1} \mathrm{kpc}\right.$; Evrard et al. 1996). These galaxies have membership probability larger than $88 \%$ as provided by the AMICO code. The contours in cyan show the extended $\mathrm{X}$-ray emission in the $0.5-2 \mathrm{keV}$ range obtained by the analysis of Chandra data in 0.5 dex surface brightness levels (for details refer to Erfanianfar et al. 2013). In the bottom right we show a zoom-in of the BCG surroundings for illustration. 1 arcmin corresponds to $\approx 230 \mathrm{kpc}$ at the clusters redshift.

(AMICO, Bellagamba et al. 2018, 2019; Maturi et al. 2019) and the Photo- $z$ Wavelet Cluster Detection Code (PZWav), as described in Euclid Collaboration (2019). AMICO and PZWav are the detection algorithms adopted for the upcoming Euclid survey (Euclid Collaboration 2019). Their use in J-PAS will allow for the results of the two surveys to be compared and crossvalidated. All cluster finder algorithms mentioned above found a minimum of $30 \%-43 \%$ of the systems detected in X-rays with $\mathrm{a} \leq 0.5 \mathrm{Mpc}$ matching scale for $z \leq 0.5$. From these first tests we can detect clusters down to a mass of $\sim 10^{13} M_{\odot}$ and up to redshift $z=0.76$ (mass based on the Chandra X-ray Luminosity scaled mass of Erfanianfar et al. 2013). The miniJPAS cluster catalog will be presented in an upcoming paper (Maturi et al., in prep.). We show here only two illustrative examples. An example of a low mass system is shown in Fig. 27. It is dominated by a bright group galaxy at $z=0.24$ and it has a mass (scaled from X-ray Luminosity) of $2.9 \times 10^{13} M_{\odot}$, typical of groups or poor clusters.

In Fig. 28, we show the most massive cluster detected in the miniJPAS footprint. This is the first cluster that stands out in all cluster finding algorithms used (VT, AMICO, PZWav). It is roughly centred on the bright cluster galaxy at RA:213.62543 Dec: +51.93786 (one of the two galaxies with spectroscopic redshift in the region, with $z_{\mathrm{s}}=0.289$ ), and we refer to this cluster as the miniJPAS Pathfinder Cluster 2470-1771, or mJPC $2470-1771^{39}$. It is near the southwest edge of the miniJPAS field and it has not been analysed previously. The cluster has an unusual central alignment that spans about $500 h_{70}^{-1} \mathrm{kpc}$ in the plane of the sky, as shown in Fig. 28. Even though this cluster was barely missed by the Chandra observations of the

\footnotetext{
${ }^{39}$ The identifier number corresponds to that of the BCG in the miniJPAS public data release. Its identifier in SDSS is SDSS J141430.10+515616.3
} 


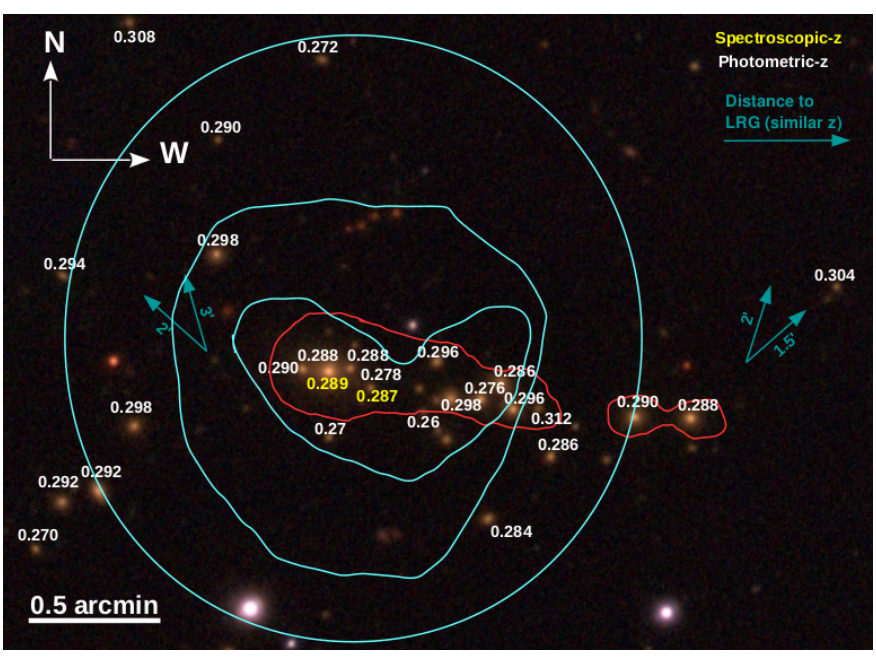

Fig. 28. Most massive cluster found in the miniJPAS footprint, centred at $\mathrm{RA}=213.6254, \mathrm{Dec}=51.9379$. This cluster is also part of the redMaPPer catalog where it is listed as a cluster with richness $\lambda=33$. The brightest galaxy has a spectroscopic redshift (from SDSS) of 0.289. Numbers in white next to galaxies indicate the photometric redshift derived from miniJPAS for galaxies with photometric redshifts between $\sim 0.26$ and 0.31 . More galaxies in this redshift interval (including some luminous ellipticals) are found at larger distances. The arrows indicate the direction and approximate distance of luminous galaxies found nearby and in this redshift interval. In red are drawn the strong lensing critical curves, estimated for fiducial sources at redshift $z_{\mathrm{s}}=2$ with the EasyCritics code applied to our data. We also show the X-ray iso-contour emission in the $0.5-2 \mathrm{keV}$ range obtained by the analysis of XMM-Newton observations (cyan) in steps of 0.5 dex in surface brightness. 1 arcmin corresponds to $\approx 200 \mathrm{kpc}$ at the cluster's redshift.

AEGIS field, the XMM-Newton program covering a large area around the Chandra field (PI Merloni) allowed us to derive some $\mathrm{X}$-ray properties relatively well. We used standard data reduction protocol using the XMM-Newton Science Analysis System (SAS) version xmmsas-v18.0.0. The X-ray spectrum of the hot gas was modeled with an absorbed thermal Bremsstrahlung with emission lines (phabs apec), with redshift fixed at 0.289, corresponding to the Bright Cluster Galaxy (BCG) and a hydrogen column density of $1.9 \times 10^{20} \mathrm{~cm}^{-2}$. Simultaneous spectral fitting of all imaging spectrometers showed an intracluster gas temperature of $(2.8 \pm 0.8) \mathrm{keV}$ for a core excised $(30 \operatorname{arcsec})$ region of 80 arcsec radius centred in the X-ray peak. The $R_{200}$, scaled from that X-ray temperature is $\sim 1200 h_{70}^{-1} \mathrm{kpc}$ and a mass $M_{200 c}$ is $\sim 3.26 \pm 1.4 \times 10^{14} M_{\odot}$. Within that radius miniJPAS finds 29 galaxies within $2000 \mathrm{~km} \mathrm{~s}^{-1}$ of the BCG redshift (scale is $4.378 \mathrm{kpc} \mathrm{arcsec}^{-1}$ ). The masses derived based on the miniJPAS data alone correspond very well to the X-ray-based mass. We derived three independent mass estimated using (i) the richness scaling relation (Lopes et al. 2009), (ii) the photo- $z$ derived velocity dispersion (Heisler et al. 1985) and (iii) the cluster model amplitude returned by the AMICO code, finding $(2.6,4.8,3.7) \times 10^{14} M_{\odot}$ with $(50 \%, 16 \%, 17 \%)$ uncertainties, respectively ${ }^{40}$. A more detailed analysis of this cluster, including the physical properties of its member galaxies, will be presented in a separate work.

\footnotetext{
${ }^{40}$ More recent spectroscopic follow-up with Gemini GMOS observations (PI:Carrasco, E.) found a velocity dispersion of $\sim 790 \mathrm{~km} \mathrm{~s}^{-1}$ and a virial mass of $(2.2 \pm 0.3) \times 10^{14} M_{\odot}$
}

\subsection{Gravitational lensing}

The excellent seeing conditions at the OAJ combined with the high quality of the J-PAS data are ideal for carrying out studies of gravitational lensing, since not only the shapes of several hundred millions of galaxies will be measured, but their redshifts will also be known with high accuracy. Strong lensing by galaxy clusters offers the opportunity to map the central distribution of dark matter in the cluster, invisible otherwise. Our team has two unique tools to automatically analyse potential cluster strong-lenses found within the J-PAS footprint. The first method was formulated by Zitrin et al. (2012), who showed that by calibrating the effective mass-to-light ratio of cluster members (e.g., using other well-known clusters analysed in HST data), their light-traces-mass method could be operated automatically on large sky surveys, without requiring multiple images as input and relying only on the photometry of identified cluster members (see also Carrasco et al. 2020). This is particularly important because multiple-image constraints for building strong-lens models are typically very hard to identify from the ground. Building on the foundations of this method, Stapelberg et al. (2019) formulated EasyCritics, the second method we use here, which not only estimates the lensing strength of clusters, but also finds them in the data. In Fig. 28 we show the critical curves from the EasyCritics method for a fiducial source at redshift $z_{\mathrm{s}}=2$. We note that the calibration used to derive these curves relied on CFHTlens data, not on the miniJPAS data, and thus these curves are for illustration only.

In addition to the possibility of J-PAS to find relatively small galaxy-cluster lenses, J-PAS offers unique opportunities to find galaxy-galaxy lenses. Most of these systems are unresolved from the ground with $2.5 \mathrm{~m}$ class telescopes, making the task to find them very difficult. However, using the J-spectra it is in principle possible to find some of these rare systems by looking for outliers corresponding to the overlapping spectra of a foreground lens (the most likely lenses are ellipticals with no emission lines) and a background high-redshift galaxy (common background sources are star forming galaxies at $z>1$, with emission lines). A similar (and successful) effort was conducted using real spectra from SDSS in the SLACS project (Auger et al. 2009)

Clusters as massive as mJPC 2470-1771 also produce weak gravitational lensing signals. The photometric depth of J-PAS, in general, makes weak lensing measurements of individual clusters challenging. However, the relatively low density of lensed background galaxies will be compensated by the large area covered by J-PAS, which will provide a large sample of lenses which can be stacked as a function of estimated mass and redshift, in order to infer parameters of both, the lenses and the background population. This has the potential to extract valuable astrophysical and cosmological information from not only massive clusters, but also from low mass ones (see e.g., Fig. 27) and even from individual galaxies.

The OAJ site has shown to have seeing better than $0.8^{\prime \prime}$ during $68 \%$ of the night time (Moles et al. 2010) and thus can produce images of sufficient quality for shear estimation from faint galaxy shape measurements. Given its area and depth, J-PAS has thus the potential for shear-based weak-lensing studies.

By applying the same procedures and selection criteria as the first year of the HSC-SSP survey (Mandelbaum et al. 2018) to miniJPAS, we find an effective number density of source galaxies of $\sim 2.7 \mathrm{arcmin}^{-2}$, using the co-added $r$ band images alone, which is a lower limit to what we expect to obtain with J-PAS. This is because the broad band images of the future J-PAS 
observations are planned to be taken under better conditions (airmass and seeing) than those of miniJPAS. Moreover, with the use of multiple filters for shape measuring we expect to significantly increase the effective number density of source galaxies.

Lensing magnification provides complementary, independent observational alternatives to gravitational shear (Broadhurst et al. 1995; Umetsu et al. 2011; Umetsu 2013). In contrast to the shear-based analysis, measuring the effects of magnification does not require source galaxies to be spatially resolved, but it does require accurate photometry across the sky and a stringent flux limit against incompleteness effects. This is where J-PAS has unique advantages against other wide-field surveys. In the presence of lensing magnification $\mu(\approx 1+2 \kappa$ in the weak-lensing regime), the source counts $n(<m)$ for a given magnitude cut $m$ is modified as $n(<m) / n_{0}(<m)=\mu^{2.5 s(m)-1}$, with $n_{0}(<m)$ the intrinsic (unlensed) source counts and $s(m)$ the intrinsic count slope, $s(m)=\mathrm{d} \log _{10} n_{0}(<m) / \mathrm{d} m$. By analysing miniJPAS data, we find that the source counts $n_{0}(<m)$ for a $r$-magnitude-limited sample of background galaxies increases progressively with magnitude down to the completeness limit $(r \sim 22.7)$. Since the net effect of magnification bias depends on the count slope $s(m)$, while its $\mathrm{S} / \mathrm{N}$ is limited by $n_{0}(<m)$, we can make use of the precise photo- $z$ delivered by J-PAS to optimize the selection of background galaxies in magnitude and redshift space so as to maximize the sensitivity. Therefore, with J-PAS data, we will have the ability to measure the magnification signal around a large statistical sample of galaxy clusters to obtain a robust mass calibration for cluster cosmology.

\section{Summary and conclusions}

In this paper, we present miniJPAS, a unique sky survey carried out with the JPAS-PF camera installed at the OAJ JST/T250 telescope together with the full J-PAS filter system. The project was designed and carried out to evaluate the scientific potential of the large J-PAS survey to follow. The miniJPAS data have allowed us to establish the photometric depth and redshift accuracy that J-PAS can achieve. This data have also allowed us to test the full reduction and analysis pipelines. The footprint of miniJPAS was chosen to cover $\sim 1 \mathrm{deg}^{2}$ of the well studied AEGIS field, in all the 56 filters of the J-PAS filter system, plus the four broad bands $u, g, r, i$. The observational strategy adopted has allowed us to reach, and often surpass, the desired minimum depths as described in Benítez et al. (2014), obtaining mag $_{\mathrm{AB}}$ between $\sim 22$ and 23.5 for the NB filters and up to 24 for the BB filters to $5 \sigma$ in a 3 arcsec aperture.

The miniJPAS primary catalog contains over $64 \mathrm{k}$ sources, extracted using the $r$ detection band with forced photometry in all other bands. In addition, over $600 \mathrm{k}$ sources have been extracted separately in the individual bands, generating catalogs that are particularly interesting for the detection of extreme line emitters over the wide spectral range covered by the narrow band filters. We estimate the full miniJPAS catalog to be complete up to $r=23.6$ for point-like sources and $r=22.7$ for extended sources. Photometric redshifts were derived using a customised version of LEPHARE, modified to work with a larger number of filters and higher resolution in redshift than typically required for broad-band photometry. We have demonstrated here that the $\mathrm{J}$ PAS filter system is able to reach sub-percent precision in photo$z$ for all sources brighter than $r \sim 22.5$. The target precision of $0.3 \%$ is reached for about half the sources, and subsamples with redshift precision as high as $0.2 \%$ can be extracted by applying appropriate cuts to the photo- $z$ outputs.
All miniJPAS data, including images, catalogs, and valueadded data products, were publicly released at the end of 2019 and made available in the J-PAS data portal ${ }^{41}$. Data can be accessed via a variety of tools: sky navigator, object list searches, and $\mathrm{ADQL}$ queries.

Our analysis of miniJPAS highlights several examples of the many scientific applications possible with the upcoming J-PAS. We show how the J-spectra can be used to characterize a very wide variety of sources, from galactic objects to high redshift quasars, thanks to the photometric detection of absorption and emission features readily visible in our spectral energy distributions. We demonstrate that stellar population studies can be carried out up to $z \sim 1$ and that the analysis of morphology and structural properties of galaxies can be enhanced by using the multi-color information. We demonstrate that J-PAS can effectively be seen as a massive IFU survey, with useful spectral resolution over an unprecedentedly large FoV. The accurate photo- $z$ achieved are key to the study of the large-scale structure, including baryon acoustic oscillations as well as the shape and evolution of the power spectrum of galaxies and clusters. It is now clear from our survey that galaxy samples with sub-percent redshift precision and a number density as high as $>10^{-2} h^{3} \mathrm{Mpc}^{-3}$ can be selected at $z<0.4$, and with a number density exceeding $10^{-4} h^{3} \mathrm{Mpc}^{-3}$ at $0.4<z<0.9$, as well as a near-complete sample of $r<23$ quasars at redshifts $z>2$. We have shown that secure identification of clusters and groups of galaxies can be made within this redshift range because our large redshift precision ensures that there is minimal contamination from non members. Furthermore, we have shown with miniJPAS that weak lensing is detectable for individual massive clusters and can be stacked for groups, allowing us to track the mass evolution of clusters to unprecedented depth, providing a self-contained evolution of the mass function with the J-PAS survey.

We conclude that miniJPAS has demonstrated the capability of the J-PAS filter system to deliver accurate classification of stars and extragalactic sources as well as the unambiguous redshift determination of tens of thousands of galaxies per squared degree. In addition, miniJPAS is only a tiny wedge of the sky that J-PAS is set to probe, as J-PAS will surpass miniJPAS by more than three orders of magnitude in terms of area and, hence, the number of sources and the volume surveyed. The full survey will provide a contiguous $3 \mathrm{D}$ map of the northern sky to $z \sim 0.9$, offering the opportunity to study galaxy evolution and the large-scale structure of the Universe over a large fraction of the cosmological volume and opening the window to serendipitous discoveries.

Acknowledgements. We acknowledge the anonymous referee for the many suggestions which helped improving the quality of the paper. This work is based on observations made with the JST/T250 telescope and PathFinder camera for the miniJPAS project at the Observatorio Astrofísico de Javalambre $(\mathrm{OAJ})$, in Teruel, owned, managed, and operated by the Centro de Estudios de Física del Cosmos de Aragón (CEFCA). We acknowledge the OAJ Data Processing and Archiving Unit (UPAD) for reducing and calibrating the OAJ data used in this work. Funding for OAJ, UPAD, and CEFCA has been provided by the Governments of Spain and Aragón through the Fondo de Inversiones de Teruel; the Aragón Government through the Research Groups E96, E103, and E16_17R; the Spanish Ministry of Science, Innovation and Universities (MCIU/AEI/FEDER, UE) with grant PGC2018-097585-B-C21; the Spanish Ministry of Economy and Competitiveness (MINECO/FEDER, UE) under AYA2015-66211-C2-1-P, AYA2015-66211-C2-2, AYA2012-30789, and ICTS2009-14; and European FEDER funding (FCDD10-4E-867, FCDD13-4E-2685) This work has made used of CEFCA's Scientific High Performance Computing system which has been funded by the Governments of Spain and Aragón

41 http://archive.cefca.es/catalogues/ minijpas-pdr201912 
through the Fondo de Inversiones de Teruel, and the Spanish Ministry of Economy and Competitiveness (MINECO-FEDER, grant AYA2012-30789). Funding for the J-PAS project has been provided also by the Brazilian agencies FINEP, FAPESP, FAPERJ and by the National Observatory of Brazil. Additional funding was also provided by the Tartu Observatory and by the Chinese Consor tium from the Academy of Sciences SB acknowledges partial support from the project PGC2018-097585-B-C22. R.A.D. acknowledges support from the Conselho Nacional de Desenvolvimento Científico e Tecnológico - CNPq through BP grant 308105/2018-4, and the Financiadora de Estudos e Projetos - FINEP grants REF. 1217/13 - 01.13.0279.00 and REF 0859/10 - 01.10.0663.00 and also FAPERJ PRONEX grant E-26/110.566/2010 for hardware funding sup port for the J-PAS project through the National Observatory of Brazil and Centro Brasileiro de Pesquisas Físicas. LRA acknowledges financial support from CNPq (306696/2018-5) and FAPESP (2015/17199-0). VM thanks CNPq (Brazil) and FAPES (Brazil) for partial financial support and the $\mathrm{H} 2020$ project No 888258. L.A.D.G. and K.U. acknowledge support from the Ministry of Science and Technology of Taiwan (grant MOST 106-2628-M-001-003-MY3) and from the Academia Sinica (grant AS-IA-107-M01). J.M.D. and D.H acknowledge the support of project PGC2018-101814-B-100. MQ thanks CNPq (Brazil) and FAPERJ (Brazil) for financial support. PC acknowledges financial sup port from FAPESP (2018/05392-8) and CNPq (310041/2018-0). AAC acknowledges support from FAPERJ (E26/203.186/2016), CNPq (304971/2016-2 an 401669/2016-5), and the Universidad de Alicante (contract UATALENTO18 02). C.Q. acknowledges support from FAPESP (2015/11442-0 and 2019/067661). V.M.P. is supported by NOIRLab, which is managed by AURA under cooperative agreement with the NSF. P.B acknowledges support from CAPES - Finance Code 001. IAA researchers acknowledge financial support from the State Agency for Research of the Spanish MCIU through the "Center of Excellence Severo Ochoa" award to the Instituto de Astrofísica de Andalucía (SEV-2017-0709). Authors acknowledge support from the Generalitat Valenciana project of excellence Prometeo/2020/085. RGD, GMS, JRM, RGB, EP acknowledge financial support from the project AyA2016-77846-P. TC is supported by the INFN INDARK PD51 and PRIN-MIUR 2015W7KAWC. MAR and ALM acknowledge support from the MINECO project FIS2016-78859 $\mathrm{P}(\mathrm{AEI} / \mathrm{FEDER}, \mathrm{UE})$. ET, AT and JL acknowledge the support by ETAg grant IUT40-2 and by EU through the ERDF CoE grant TK133 and MOBTP86. CK, JMV, JIP acknowledge financial support from project AYA2016-79724C4-4P. PAAL thanks the support of CNPq (309398/2018-5). LC thanks CNPq for partial support. Y.J-T acknowledges financial support from the FAPERJ (E 26/202.835/2016), and from the Horizon 2020 Marie Sklodowska-Curie gran agreement No 898633. DMD acknowledges financial support from the SFB 881 of the DFG and from the MINECO grant AYA2016-81065-C2-2. FP acknowledges support of the project PGC2018-101931-B-I00. JC acknowledges support of the project E AYA2017-88007-C3-1-P, and co-financed by the FEDER JIGs acknowledges support of projects of reference AYA2017-88007-C3-3-P and PGC2018-099705-B-I00 and co-financed by the FEDER. EMG and PV would like to acknowledge financial support from the project ESP2017-83921 C2-1-R. GMS acknowleges financial support from a predoctoral contract, ref PRE2018-085523 (MCIU/AEI/FSE, UE). S.C. is partially supported by CNPq R.G.L. acknowledges CAPES (process 88881.162206/2017-01) and Alexande von Humboldt Foundation for the financial support. JSA acknowledges support from FAPERJ (E26/203.024/2017), CNP (310790/2014-0 and 400471/2014-0) and FINEP (1217/13 - 01.13.0279.00 and Ref. 0859/10 - 01.10.0663.00). RvM acknowledges support from CNPq. AFS, PAM, VJM and FJB acknowledge support from project AYA2016-81065-C2-2. PAM acknowledges support from the "Subprograma Atracció de Talent - Contractes Postdoctorals de la Universitat de Valéncia". ESC acknowledges support from CNPq (308539/2018 4) and FAPESP (2019/19687-2). CMdO acknowledges support from CNPq (grant 312333/2014-5) and FAPESP (grant 2009/54202-8). LSJ acknowledges support from CNPq (grant 304819/2017-4) and FAPESP (grant 2012/008004). JMC acknowledges support from CNPq (grant 310727/2016-2). C.H.-M and N. Greisel also acknowledge the support of the European Union via the Career Integration Grant CIG-PCIG9-GA-2011-294183. JJBP and AMC would like to acknowledge the support from the grant PGC2018-094626-B-C21 an the Basque Government grant IT-979-16. AMC acknowledges the postdoctora contract from the University of the Basque Country UPV/EHU "Especializacioón de personal investigador doctor" program. MLLD acknowledges CAPES

- Finance Code 001; and CNPq (142294/2018-7). GB acknowledges financia support from the UNAM through grant DGAPA/PAPIIT IG100319, from CONACyT through grant CB2015-252364, and from FAPESP project 2017/02375- 2 and 2018/05392-8. M.J. Rebouças acknowledges the support of FAPERJ under a CNE E-26/202.864/2017 grant, and CNPq. Support by CNPq (305409/2016-6) and FAPERJ (E-26/202.841/2017) is acknowledged by DL. AB acknowledges a CNPq fellowship. C.A.G.acknowledges support from CAPES. EA acknowledges support from FAPESP (2011/18729-1). AC acknowledges support from PNPD/CAPES. ABA and FSK acknowledge the Severo Ochoa program SEV-2015-0548. FSK also thanks the AYA2017-89891-P and the RYC2015-18693 grants. DF acknowledges support from the Atracción del
Talento Científico en Salamanca programme and the project PGC2018-096038B-I00. SDSS - This research has made use of SDSS, which is managed by the Astrophysical Research Consortium for the Participating Institutions of the SDSS Collaboration including the Brazilian Participation Group, the Carnegie Institution for Science, Carnegie Mellon University, the Chilean Participation Group, the French Participation Group, Harvard-Smithsonian Center for Astrophysics, Instituto de Astrofísica de Canarias, The Johns Hopkins University, Kavli Institute for the Physics and Mathematics of the Universe (IPMU), University of Tokyo, the Korean Participation Group, Lawrence Berkeley National Laboratory, Leibniz Institut für Astrophysik Potsdam (AIP), Max-Planck-Institut für Astronomie (MPIA Heidelberg), Max-Planck-Institut für Astrophysik (MPA Garching), Max-Planck-Institut für Extraterrestrische Physik (MPE), National Astronomical Observatories of China, New Mexico State University, New York University, University of Notre Dame, Observatório Nacional/MCTI, The Ohio State University, Pennsylvania State University, Shanghai Astronomical Observatory, United Kingdom Participation Group, Universidad Nacional Autónoma de México, University of Arizona, University of Colorado Boulder, University of Oxford, University of Portsmouth, University of Utah, University of Virginia, University of Washington, University of Wisconsin, Vanderbilt University, and Yale University. ADS - This research has made use of NASA's Astrophysics Data System. HEASARC - This research has made use of data and/or software provided by the High Energy Astrophysics Science Archive Research Center (HEASARC), which is a service of the Astrophysics Science Division at NASA/GSFC. This research has made use of the WEB Cosmology Calculator Wright (2006, PASP, 118,1711 ) DEEP - Funding for the DEEP2 Galaxy Redshift Survey has been provided by NSF grants AST-95-09298, AST-0071048, AST-0507428, and AST0507483 as well as NASA LTSA grant NNG04GC89G. This paper made use of the Hyper Suprime-Cam (HSC), which includes the astronomical communities of Japan and Taiwan, and Princeton University. The HSC instrumentation and software were developed by the National Astronomical Observatory of Japan (NAOJ), the Kavli Institute for the Physics and Mathematics of the Universe (Kavli IPMU), the University of Tokyo, the High Energy Accelerator Research Organization (KEK), the Academia Sinica Institute for Astronomy and Astrophysics in Taiwan (ASIAA), and Princeton University. Funding was contributed by the FIRST program from Japanese Cabinet Office, the Ministry of Education, Culture, Sports, Science and Technology (MEXT), the Japan Society for the Promotion of Science (JSPS), Japan Science and Technology Agency (JST), the Toray Science Foundation, NAOJ, Kavli IPMU, KEK, ASIAA, and Princeton University. This research made use of Python (www . python . org) and several Python packages like Numpy; Astropy (http://www.astropy.org) a community-developed core Python package for Astronomy; matplotlib; IPython Cython. This work has made use of data from the European Space Agency (ESA) mission Gaia (https://www. cosmos.esa.int/gaia), processed by the Gaia Data Processing and Analysis Consortium (DPAC, https : //www . cosmos . esa . int/web/gaia/dpac/consortium). Funding for the DPAC has been provided by national institutions, in particular the institutions participating in the Gaia Multilateral Agreement.

\section{References}

Abolfathi, B., Aguado, D. S., Aguilar, G., et al. 2018, ApJS, 235, 42 Abramo, L. R., \& Bertacca, D. 2017, Phys. Rev. D, 96, 123535 Abramo, L. R., Strauss, M. A., Lima, M., et al. 2012, MNRAS, 423, 3251 Abramo, L. R., Secco, L. F., \& Loureiro, A. 2016, MNRAS, 455, 3871 Aihara, H., Arimoto, N., Armstrong, R., et al. 2018, PASJ, 70, S4 Aihara, H., AlSayyad, Y., Ando, M., et al. 2019, PASJ, 71, 114 Alam, S., Albareti, F. D., Allende Prieto, C., et al. 2015, ApJS, 219, 12 Allen, S. W., Evrard, A. E., \& Mantz, A. B. 2011, ARA\&A, 49, 409 Andersen, M. I., Freyhammer, L., \& Storm, J. 1995, in Calibrating and Understanding HST and ESO Instruments, European Southern Observatory Conference and Workshop Proceedings, 53, 87

Arnalte-Mur, P., Martínez, V. J., Norberg, P., et al. 2014, MNRAS, 441, 1783 Arnouts, S., \& Ilbert, O. 2011, Astrophysics Source Code Library [record ascl:1108.009]

Ascaso, B., Wittman, D., \& Benítez, N. 2012, MNRAS, 420, 1167

Ascaso, B., Benítez, N., Dupke, R., et al. 2016, MNRAS, 456, 4291

Auger, M. W., Treu, T., Bolton, A. S., et al. 2009, ApJ, 705, 1099

Balogh, M. L., Morris, S. L., Yee, H. K. C., Carlberg, R. G., \& Ellingson, E. 1999, ApJ, 527, 54

Baqui, P. O., Marra, V., Casarini, L., et al. 2021, A\&A, 645, A87

Bellagamba, F., Roncarelli, M., Maturi, M., \& Moscardini, L. 2018, MNRAS, 473, 5221

Bellagamba, F., Sereno, M., Roncarelli, M., et al. 2019, MNRAS, 484, 1598

Belli, S., Newman, A. B., \& Ellis, R. S. 2015, ApJ, 799, 206

Benítez, N. 2000, ApJ, 536, 571

Benítez, N., Moles, M., Aguerri, J. A. L., et al. 2009, ApJ, 692, L5 
Benítez, N., Dupke, R., Moles, M., et al. 2014, ArXiv e-prints [arXiv:1403.5237]

Bernstein, G. M., Abbott, T. M. C., Desai, S., et al. 2017, PASP, 129, 114502 Bertin, E. 2010a, Astrophysics Source Code Library [record ascl:1010.063] Bertin, E. 2010b, Astrophysics Source Code Library [record ascl:1010. 068] Bertin, E. 2011, ASP Conf. Ser., 442, 435

Bertin, E., \& Arnouts, S. 1996, A\&AS, 117, 393

Betoule, M., Marriner, J., Regnault, N., et al. 2013, A\&A, 552, A124

Bianchi, L., Herald, J., Efremova, B., et al. 2011, Ap\&SS, 335, 161

Blake, C., \& Glazebrook, K. 2003, ApJ, 594, 665

Bleem, L. E., Bocquet, S., Stalder, B., et al. 2020, ApJS, 247, 25

Boch, T., Fitzpatrick, M., Taylor, M., et al. 2012, Simple Application Messaging Protocol Version 1.3, IVOA Recommendation 11 April 2012

Bonatto, C., Chies-Santos, A. L., Coelho, P. R. T., et al. 2019, A\&A, 622, A179

Bottrell, C., Simard, L., Mendel, J. T., \& Ellison, S. L. 2019, MNRAS, 486, 390

Bower, R. G., McCarthy, I. G., \& Benson, A. J. 2008, MNRAS, 390, 1399

Brammer, G. B., Whitaker, K. E., van Dokkum, P. G., et al. 2009, ApJ, 706, L173

Brauneck, U., Sprengard, R., Bourquin, S., \& Marín-Franch, A. 2018a, J. Astron. Telesc. Instrum. Syst., 4, 015002

Brauneck, U., Sprengard, R., Bourquin, S., \& Marín-Franch, A. 2018b, J. Astron. Telesc. Instrum. Syst., 4, 015003

Broadhurst, T. J., Taylor, A. N., \& Peacock, J. A. 1995, ApJ, 438, 49

Bruzual, G., \& Charlot, S. 2003, MNRAS, 344, 1000

Bundy, K., Bershady, M. A., Law, D. R., et al. 2015, ApJ, 798, 7

Capak, P., Cowie, L. L., Hu, E. M., et al. 2004, AJ, 127, 180

Cardamone, C., Schawinski, K., Sarzi, M., et al. 2009, MNRAS, 399, 1191

Cardamone, C. N., Urry, C. M., Schawinski, K., et al. 2010, ApJ, 721, L38

Carrasco, M., Zitrin, A., \& Seidel, G. 2020, MNRAS, 491, 3778

Cenarro, A. J., Moles, M., Marín-Franch, A., et al. 2014, SPIE Conf. Ser., 9149, 91491I

Cenarro, A. J., Ederoclite, A., Íñiguez, C., et al. 2018, Proc. SPIE, 10700, 107000D

Cenarro, A. J., Moles, M., Cristóbal-Hornillos, D., et al. 2019, A\&A, 622, A176

Chambers, K. C., Magnier, E. A., Metcalfe, N., et al. 2016, ArXiv e-prints [arXiv:1612.05560]

Chaves-Montero, J., Bonoli, S., Salvato, M., et al. 2017, MNRAS, 472, 2085

Chaves-Montero, J., Angulo, R. E., \& Hernández-Monteagudo, C. 2018, MNRAS, 477, 3892

Chiti, A., Frebel, A., Jerjen, H., Kim, D., \& Norris, J. E. 2020, ApJ, 891, 8

Cid Fernandes, R., Mateus, A., Sodré, L., Stasińska, G., \& Gomes, J. M. 2005, MNRAS, 358, 363

Civera, T., \& Hernandez, J. 2020, ASP Conf. Ser., 524, 999

Coil, A. L., Newman, J. A., Kaiser, N., et al. 2004, ApJ, 617, 765

Conselice, C. J. 2003, ApJS, 147, 1

Costa, A. A., Marcondes, R. J. F., Landim, R. G., et al. 2019, MNRAS, 488, 78

Cristóbal-Hornillos, D., Varela, J., Ederoclite, A., et al. 2014, SPIE Conf. Ser. 9152, 915200

Cutri, R. M., Wright, E. L., Conrow, T., et al. 2014, VizieR Online Data Catalog:II/328

Dai, X., Bregman, J. N., Kochanek, C. S., \& Rasia, E. 2010, ApJ, 719, 119

Davies, J. I., Baes, M., Bianchi, S., et al. 2017, PASP, 129, 044102

Davis, M., Guhathakurta, P., Konidaris, N. P., et al. 2007, ApJ, 660, L1

de Amorim, A. L., García-Benito, R., Cid Fernandes, R., et al. 2017, MNRAS, 471,3727

DES Collaboration (Abbott, T., et al.) 2020, Phys. Rev. D, 102, 023509

DESI Collaboration (Aghamousa, A., et al.) 2016, ArXiv e-prints [arXiv:1611.00036]

Díaz-García, L. A., Cenarro, A. J., López-Sanjuan, C., et al. 2015, A\&A, 582, A14

Díaz-García, L. A., Cenarro, A. J., López-Sanjuan, C., et al. 2019a, A\&A, 631, A156

Díaz-García, L. A., Cenarro, A. J., López-Sanjuan, C., et al. 2019b, A\&A, 631, A157

Díaz-García, L. A., Cenarro, A. J., López-Sanjuan, C., et al. 2019c, A\&A, 631, A158

Dowler, P., Bonnarel, F., \& Tody, D. 2015, IVOA Simple Image Access Version 2.0, IVOA Recommendation 23 December 2015

Dowler, P., Rixon, G., \& Tody, D. 2010, Table Access Protocol Version 1.0 IVOA Recommendation 27 March 2010

Driver, S. P., Hill, D. T., Kelvin, L. S., et al. 2011, MNRAS, 413, 971

Elbaz, D., Daddi, E., Le Borgne, D., et al. 2007, A\&A, 468, 33

Erfanianfar, G., Finoguenov, A., Tanaka, M., et al. 2013, ApJ, 765, 117

Eriksen, M., Alarcon, A., Gaztanaga, E., et al. 2019, MNRAS, 484, 4200

Euclid Collaboration (Adam, R., et al.) 2019, A\&A, 627, A23

Evrard, A. E., Metzler, C. A., \& Navarro, J. F. 1996, ApJ, 469, 494

Fernique, P., Boch, T., Donaldson, T., et al. 2019, MOC - HEALPix Multi-Order Coverage map Version 1.1, IVOA Recommendation 07 October 2019

Ferrari, F., de Carvalho, R. R., \& Trevisan, M. 2015, ApJ, 814, 55
Fitzpatrick, E. L. 1999, PASP, 111, 63

Gaia Collaboration (Brown, A. G. A., et al.) 2018, A\&A, 616, A1

García-Benito, R., Zibetti, S., Sánchez, S. F., et al. 2015, A\&A, 576, A135

Girardi, L., Groenewegen, M. A. T., Hatziminaoglou, E., \& da Costa, L. 2005, A\&A, 436, 895

Gong, Y., Cooray, A., Silva, M. B., Santos, M. G., \& Lubin, P. 2011, ApJ, 728, L46

González Delgado, R. M., García-Benito, R., Pérez, E., et al. 2015, A\&A, 581, A103

González Delgado, R. M., Díaz-García, L. A., de Amorim, A., et al. 2021, A\&A, 649, A79

Green, G. M., Schlafly, E. F., Finkbeiner, D., et al. 2018, MNRAS, 478, 651

Grogin, N. A., Kocevski, D. D., Faber, S. M., et al. 2011, ApJS, 197, 35

Gurung-López, S., Orsi, Á. A., Bonoli, S., Baugh, C. M., \& Lacey, C. G. 2019, MNRAS, 486, 1882

Gurung-López, S., Orsi, Á. A., Bonoli, S., et al. 2020, MNRAS, 491, 3266

Gutiérrez-Soto, L. A., Gonçalves, D. R., Akras, S., et al. 2020, A\&A, 633, A123

Heckman, T. M., Hoopes, C. G., Seibert, M., et al. 2005, ApJ, 619, L35

Heisler, J., Tremaine, S., \& Bahcall, J. N. 1985, ApJ, 298, 8

Hernandez, J., \& Civera, T. 2020, ASP Conf. Ser., 524, 999

Hernández-Monteagudo, C., Chaves-Montero, J., \& Angulo, R. E. 2021a, MNRAS, 503, L56

Hernández-Monteagudo, C., Chaves-Montero, J., Angulo, R. E., \& Ariccò, G. 2021b, MNRAS, 503, L62

Hickson, P., Gibson, B. K., \& Callaghan, K. A. S. 1994, MNRAS, 267, 911

Hill, G. J., Gebhardt, K., Komatsu, E., et al. 2008, ASP Conf. Ser., 399, 115

Hilton, M., Hasselfield, M., Sifón, C., et al. 2018, ApJS, 235, 20

Høg, E., Fabricius, C., Makarov, V. V., et al. 2000, A\&A, 355, L27

Huang, J.-S., Thompson, D., Kümmel, M. W., et al. 2001, A\&A, 368, 787

Huang, S., Leauthaud, A., Murata, R., et al. 2018, PASJ, 70, S6

Hurtado-Gil, L., Arnalte-Mur, P., Martínez, V. J., et al. 2016, ApJ, 818, 174

Ivezić, Ž., Kahn, S. M., Tyson, J. A., et al. 2019, ApJ, 873, 111

Jiménez-Teja, Y., Dupke, R., Benítez, N., et al. 2018, ApJ, 857, 79

Jiménez-Teja, Y., Dupke, R. A., Lopes de Oliveira, R., et al. 2019, A\&A, 622, A183

Kashikawa, N., Shimasaku, K., Yasuda, N., et al. 2004, PASJ, 56, 1011

Kennedy, R., Bamford, S. P., Häußler, B., et al. 2016, A\&A, 593, A84

Kitaura, F.-S., Ata, M., Rodriguez-Torres, S. A., et al. 2021, MNRAS, 503, 3456

Kümmel, M. W., \& Wagner, S. J. 2001, A\&A, 370, 384

Laigle, C., McCracken, H. J., Ilbert, O., et al. 2016, ApJS, 224, 24

Law, D. R., Yan, R., Bershady, M. A., et al. 2015, AJ, 150, 19

Lilly, S. J., Le Fevre, O., Crampton, D., Hammer, F., \& Tresse, L. 1995, ApJ, 455,50

Loaiza-Agudelo, M., Overzier, R. A., \& Heckman, T. M. 2020, ApJ, 891, 19

Logroño-García, R., Vilella-Rojo, G., López-Sanjuan, C., et al. 2019, A\&A, 622, A180

Longeard, N., Martin, N., Starkenburg, E., et al. 2018, MNRAS, 480, 2609

Longeard, N., Martin, N., Starkenburg, E., et al. 2020, MNRAS, 491, 356

Lopes, P. A. A., de Carvalho, R. R., Gal, R. R., et al. 2004, AJ, 128, 1017

Lopes, P. A. A., de Carvalho, R. R., Kohl-Moreira, J. L., \& Jones, C. 2009, MNRAS, 399, 2201

López Fernández, R., Cid Fernandes, R., González Delgado, R. M., et al. 2016, MNRAS, 458, 184

López-Sanjuan, C., Varela, J., Cristóbal-Hornillos, D., et al. 2019a, A\&A, 631, A119

López-Sanjuan, C., Vázquez Ramió, H., Varela, J., et al. 2019b, A\&A, 622, A177 LSST Science Collaboration (Abell, P. A., et al.) 2009, ArXiv e-prints [arXiv:0912.0201]

Lucatelli, G., \& Ferrari, F. 2019, MNRAS, 489, 1161

Magris, C. G., Mateu, P. J., Mateu, C., et al. 2015, PASP, 127, 16

Mandelbaum, R., Miyatake, H., Hamana, T., et al. 2018, PASJ, 70, S25

Manfroid, J. 1995, A\&AS, 113, 587

Marin-Franch, A., Taylor, K., Cepa, J., et al. 2012, SPIE Conf. Ser., 8446, $84466 \mathrm{H}$

Marín-Franch, A., Taylor, K., Santoro, F. G., et al. 2017, in Highlights on Spanish Astrophysics IX, eds. S. Arribas, A. Alonso-Herrero, F. Figueras, C. Hernández-Monteagudo, A. Sánchez-Lavega, \& S. Pérez-Hoyos, 670

Martín-Navarro, I., Pérez-González, P. G., Trujillo, I., et al. 2015, ApJ, 798, L4

Martínez-Solaeche, G., González Delgado, R. M., García-Benito, R., et al. 2021, A\&A, 647, A158

Mathis, H., Charlot, S., \& Brinchmann, J. 2006, MNRAS, 365, 385

Matthews, D. J., Newman, J. A., Coil, A. L., Cooper, M. C., \& Gwyn, S. D. J. 2013, ApJS, 204, 21

Maturi, M., Bellagamba, F., Radovich, M., et al. 2019, MNRAS, 485, 498

McDonald, P., \& Seljak, U. 2009, JCAP, 2009, 007

Mejía-Narváez, A., Bruzual, G., Magris, C. G., et al. 2017, MNRAS, 471, 4722

Mendes de Oliveira, C., Ribeiro, T., Schoenell, W., et al. 2019, MNRAS, 489, 241 
Merloni, A., Predehl, P., Becker, W., et al. 2012, ArXiv e-prints [arXiv:1209.3114]

Merson, A. I., Baugh, C. M., Helly, J. C., et al. 2013, MNRAS, 429, 556

Moles, M., Benítez, N., Aguerri, J. A. L., et al. 2008, AJ, 136, 1325

Moles, M., Sánchez, S. F., Lamadrid, J. L., et al. 2010, PASP, 122, 363

Molino, A., Benítez, N., Moles, M., et al. 2014, MNRAS, 441, 2891

Molino, A., Benítez, N., Ascaso, B., et al. 2017, MNRAS, 470, 95

Molino, A., Costa-Duarte, M. V., Mendes de Oliveira, C., et al. 2019, A\&A, 622, A178

Montero-Dorta, A. D., Abramo, L. R., Granett, B. R., de la Torre, S., \& Guzzo, L. 2020, MNRAS, 493, 5257

Moresco, M., Pozzetti, L., Cimatti, A., et al. 2013, A\&A, 558, A61

Moustakas, J., Coil, A. L., Aird, J., et al. 2013, ApJ, 767, 50

Newman, J. A., Cooper, M. C., Davis, M., et al. 2013, ApJS, 208, 5

Oke, J. B., \& Gunn, J. E. 1983, ApJ, 266, 713

Osuna, P., Ortiz, I., Lusted, J., et al. 2008, IVOA Astronomical Data Query Language Version 2.00, IVOA Recommendation 30 October 2008

Ouchi, M., Harikane, Y., Shibuya, T., et al. 2018, PASJ, 70, S13

Overzier, R. A., Heckman, T. M., Schiminovich, D., et al. 2010, ApJ, 710, 979

Padilla, C., Castander, F. J., Alarcón, A., et al. 2019, AJ, 157, 246

Palanque-Delabrouille, N., Magneville, C., Yèche, C., et al. 2016, A\&A, 587, A41

Pâris, I., Petitjean, P., Aubourg, É., et al. 2018, A\&A, 613, A51

Paulino-Afonso, A., Sobral, D., Darvish, B., et al. 2020, A\&A, 633, A70

Peng, C. Y., Ho, L. C., Impey, C. D., \& Rix, H. W. 2011, Astrophysics Source Code Library [record ascl:1104.010]

Pérez-González, P. G., Cava, A., Barro, G., et al. 2013, ApJ, 762, 46

Pieri, M. M., Bonoli, S., Chaves-Montero, J., et al. 2016, in SF2A-2016: Proceedings of the Annual meeting of the French Society of Astronomy and Astrophysics, eds. C. Reylé, J. Richard, L. Cambrésy, et al., 259

Placco, V. M., Beers, T. C., Santucci, R. M., et al. 2018, AJ, 155, 256

Placco, V. M., Santucci, R. M., Beers, T. C., et al. 2019, ApJ, 870, 122

Planck Collaboration VI. 2020, A\&A, 641, A6

Plante, R., Williams, R., Hanisch, R., \& Szalay, A. 2008, Simple Cone Search Version 1.03 IVOA Recommendation 22 February 2008

Ramella, M., Boschin, W., Fadda, D., \& Nonino, M. 2001, A\&A, 368, 776

Resco, M. A., \& Maroto, A. L. 2021, JCAP, 01, 021

Resco, M. A., Antonio, L. M., Jailson, S. A., et al. 2020, MNRAS, 493, 3616

Roman-Oliveira, F. V., Chies-Santos, A. L., Rodríguez del Pino, B., et al. 2019 , MNRAS, 484, 892

Sánchez, S. F., Kennicutt, R. C., Gil de Paz, A., et al. 2012, A\&A, 538, A8

Sánchez, S. F., García-Benito, R., Zibetti, S., et al. 2016, A\&A, 594, A36

Sandage, A., Tammann, G. A., \& Yahil, A. 1979, ApJ, 232, 352

San Roman, I., Cenarro, A. J., Díaz-García, L. A., et al. 2018, A\&A, 609, A20

San Roman, I., Sánchez-Blázquez, P., Cenarro, A. J., et al. 2019, A\&A, 622 A181

Santucci, R. M., Placco, V. M., Rossi, S., et al. 2015a, ApJ, 801, 116

Santucci, R. M., Beers, T. C., Placco, V. M., et al. 2015b, ApJ, 813, L16

Schlafly, E. F., Meisner, A. M., Stutz, A. M., et al. 2016, ApJ, 821, 78

Scoville, N., Aussel, H., Brusa, M., et al. 2007, ApJS, 172, 1

Seljak, U. 2009, Phys. Rev. Lett., 102, 021302

Seo, H.-J., \& Eisenstein, D. J. 2003, ApJ, 598, 720

Shipp, N., Drlica-Wagner, A., Balbinot, E., et al. 2018, ApJ, 862, 114

Shipp, N., Li, T. S., Pace, A. B., et al. 2019, ApJ, 885, 3

Spinoso, D., Orsi, A., López-Sanjuan, C., et al. 2020, A\&A, 643, A149

Stapelberg, S., Carrasco, M., \& Maturi, M. 2019, MNRAS, 482, 1824

Starkenburg, E., Youakim, K., Martin, N., et al. 2019, MNRAS, 490, 5757

Sunyaev, R. A., \& Zeldovich, Y. B. 1972, Comments Astrophys. Space Phys., 4, 173

Swanson, M. E. C., Tegmark, M., Hamilton, A. J. S., \& Hill, J. C. 2008, MNRAS, 387,1391

Taylor, K., Marín-Franch, A., Laporte, R., et al. 2014, J. Astron. Instrum., 3, 1350010

Tempel, E., Stoica, R. S., Martínez, V. J., et al. 2014, MNRAS, 438, 3465

Umetsu, K. 2013, ApJ, 769, 13

Umetsu, K., Broadhurst, T., Zitrin, A., Medezinski, E., \& Hsu, L.-Y. 2011, ApJ 729,127

Vika, M., Bamford, S. P., Häußler, B., et al. 2013, MNRAS, 435, 623

Vika, M., Vulcani, B., Bamford, S. P., Häußler, B., \& Rojas, A. L. 2015, A\&A, 577, A97

Vilella-Rojo, G., Viironen, K., López-Sanjuan, C., et al. 2015, A\&A, 580, A47

Vulcani, B., Bamford, S. P., Häußler, B., et al. 2014, MNRAS, 441, 1340

Walcher, J., Groves, B., Budavári, T., \& Dale, D. 2011, Ap\&SS, 331, 1

Wang, J., Xu, W., Lee, B., et al. 2020, ApJ, 903, 103

Wester, W., \& Dark Energy Survey Collaboration 2005, in Dark Energy Survey and Camera, eds. S. C. Wolff, \& T. R. Lauer, ASP Conf. Ser., 339, 152

Whitten, D. D., Beers, T. C., Placco, V. M., et al. 2019a, ApJ, 884, 67

Whitten, D. D., Placco, V. M., Beers, T. C., et al. 2019b, A\&A, 622, A182
Wolf, C., Meisenheimer, K., Rix, H. W., et al. 2003, A\&A, 401, 73 Yasuda, N., Fukugita, M., Narayanan, V. K., et al. 2001, AJ, 122, 1104 Yip, C. W., Connolly, A. J., Vanden Berk, D. E., et al. 2004, AJ, 128, 2603 Yoon, J., Beers, T. C., Dietz, S., et al. 2018, ApJ, 861, 146

York, D. G., Adelman, J., Anderson, J. E., Jr., et al. 2000, AJ, 120, 1579

Youakim, K., Starkenburg, E., Aguado, D. S., et al. 2017, MNRAS, 472, 2963

Zitrin, A., Broadhurst, T., Bartelmann, M., et al. 2012, MNRAS, 423, 2308

1 Centro de Estudios de Física del Cosmos de Aragón (CEFCA), Plaza San Juan, 1, 44001 Teruel, Spain

2 Donostia International Physics Center (DIPC), Manuel Lardizabal Ibilbidea, 4, San Sebastián, Spain e-mail: silvia.bonoli@dipc.org

3 Ikerbasque, Basque Foundation for Science, 48013 Bilbao, Spain

4 Centro de Estudios de Física del Cosmos de Aragón (CEFCA), Unidad Asociada al CSIC, Plaza San Juan, 1, 44001 Teruel, Spain

5 Instituto de Física, Universidade de São Paulo, Rua do Matão 1371, CEP 05508-090, São Paulo, Brazil

6 Observatório Nacional, Ministério da Ciencia, Tecnologia, Inovação e Comunicações, Rua General José Cristino, 77, São Cristóvão, 20921-400 Rio de Janeiro, Brazil

7 Instituto de Astrofísica de Andalucía - CSIC, Apdo 3004, 18080 Granada, Spain

8 Nũcleo de Astrofísica e Cosmologia, PPGCosmo \& Dep. de Física, Universidade Federal do Espírito Santo, Vitória 29075-910, ES, Brazil

9 Departamento de Física, Universidade Federal de Sergipe, Av. Marechal Rondon, s/n, 49000-000 São Cristóvão, SE, Brazil

10 Instituto de Física, Universidade Federal da Bahia, 40210-340 Salvador, BA, Brazil

11 Academia Sinica Institute of Astronomy \& Astrophysics (ASIAA), $11 \mathrm{~F}$ of Astronomy-Mathematics Building, AS/NTU, No. 1, Section4, Roosevelt Road, Taipei 10617, Taiwan

12 Instituto de Física de Cantabria (CSIC-UC), Avda. Los Castros s/n, 39005 Santander, Spain

13 Departamento de Astronomia, Instituto de Astronomia, Geofísica e Ciências Atmosféricas, Universidade de São Paulo, São Paulo, Brazil

14 Instituto de Matematica Estatistica e Fisica, Universidade Federal do Rio Grande (IMEF-FURG), Rio Grande, RS, Brazil

15 Campus Duque de Caxias, Universidade Federal do Rio de Janeiro, 25265-970 Duque de Caxias, RJ, Brazil

16 Department of Physics, Ben-Gurion University of the Negev, Be'erSheva 84105, Israel

17 Community Science and Data Center/NSF's NOIRLab, $950 \mathrm{~N}$. Cherry Ave., Tucson, AZ 85719, USA

18 Instituto de Física, Universidade Federal do Rio de Janeiro, 21941972 Rio de Janeiro, RJ, Brazil

19 Observatório do Valongo, Universidade Federal do Rio de Janeiro, 20080-090 Rio de Janeiro, RJ, Brazil

20 Department of Astronomy, Beijing Normal University, Beijing 100875, PR China

21 Instituto Universitario de Física Aplicada a las Ciencias y las Tecnologías, Universidad de Alicante, San Vicent del Raspeig, 03080 Alicante, Spain

22 Departamento de Física Teórica and Instituto de Física de Partículas y del Cosmos, IPARCOS, Universidad Complutense de Madrid, 28040 Madrid, Spain

23 Departamento de Física - CFM - Universidade Federal de Santa Catarina, Florianópolis, SC, Brazil

24 Dipartimento di Fisica, Sezione di Astronomia, Universitá di Trieste, Via Tiepolo 11, 34143 Trieste, Italy

25 INAF - Osservatorio Astronomico di Trieste, Via Tiepolo 11, 34131 Trieste, Italy

26 IFPU - Institute for Fundamental Physics of the Universe, Via Beirut 2, 34151 Trieste, Italy

27 INFN - Sezione di Trieste, 34100 Trieste, Italy

28 Tartu Observatory, University of Tartu, Observatooriumi 1, 61602 Tõravere, Estonia 
29 European Southern Observatory (ESO), Alonso de Córdova 3107, Vitacura, Santiago, Chile

30 Department of Astronomy, University of Michigan, 311 West Hall, 1085 South University Ave., Ann Arbor, USA

31 Department of Physics and Astronomy, University of Alabama, Box 870324, Tuscaloosa, AL, USA

32 Instituto de Física, Universidade de Brasília, Caixa Postal 04455, Brasília, DF 70919-970, Brazil

33 Instruments4, 4121 Pembury Place, La Canada Flintridge, CA 91011, USA

34 Zentrum für Astronomie, Universität Heidelberg, Philosophenweg 12, 69120 Heidelberg, Germany

35 Institut für Theoretische Physik, Universität Heidelberg, Philosophenweg 16, 69120 Heidelberg, Germany

36 Department of Physics, University of Helsinki, Gustaf Hällströmin katu 2, 00014 Helsinki, Finland

37 Physik-Department, Technische Universität München, JamesFranck-Strasse, 85748 Garching, Germany

38 Unidad Asociada "Grupo de Astrofísica Extragaláctica y Cosmología", IFCA-CSIC/Universitat de València, València, Spain

39 Department of Theoretical Physics, University of the Basque Country, UPV/EHU, 48080 Bilbao, Spain

40 Departamento de Astrofísica, Universidad de La Laguna, 38206 La Laguna Tenerife, Spain

${ }^{41}$ Instituto de Astrofísica de Canarias, 38200 La Laguna, Tenerife, Spain

42 LERMA, Observatoire de Paris, PSL Research University, CNRS, Sorbonne Universités, UPMC Univ. Paris 06, 75014 Paris, France

43 Univeristé de Paris, 5 Rue Thomas Mann, 75013 Paris, France

44 Departamento de Astronomia, Instituto de Física, Universidade Federal do Rio Grande do Sul (UFRGS), Av. Bento Gonçalves 9500, Porto Alegre, RS, Brazil

45 Departamento de Física Teórica e Experimental, Universidade Federal do Rio Grande do Norte, 59072-970 Natal, RN, Brazil

46 Physics Department, University of Missouri Science and Technology, 1315 N Pine St., 65409 Rolla, MO, USA
47 Gemini Observatory/NSF's NOIRLab, Casilla 603, La Serena, Chile

48 Departamento de Física, Matemática e Computação, Universidade do Estado do Rio de Janeiro, Rod. Pres. Dutra, 27537-000 Resende, RJ, Brazil

49 Observatori Astronómic de la Universitat de Valéncia, Ed. Instituts d'Investigació, Parc Científic. C/ Catedrático José Beltran, n2, 46980 Paterna, Valencia, Spain

50 Departament d'Astronomia i Astrofísica, Universitat de Valéncia, 46100 Burjassot, Spain

51 Centro de Investigaciones de Astronomía, CIDA, Mérida, Venezuela

52 Instituto de Radioastronomía y Astrofísica, UNAM, Campus Morelia, Michoacán 58089, Mexico

53 Department of Astronomy, University of Florida, 211 Bryant Space Center, Gainesville, FL 32611, USA

54 APC, AstroParticule et Cosmologie, Université Paris Diderot, CNRS/IN2P3, CEA/Irfu, Observatoire de Paris, Sorbonne Paris Cite, 75205 Paris Cedex 13, France

55 Department of Physics, University of Hong Kong, Pokfulam Road, Hong Kong

56 Centro Brasileiro de Pesquisas Físicas, Department of Theoretical Physics, Rua Dr. Xavier Sigaud, 150, Rio de Janeiro, RJ 22290-180, Brazil

${ }^{57}$ Institute of Physics, University of Szczecin, Wielkopolska 15, 70451 Szczecin, Poland

58 Dipartimento di Fisica “E. Pancini”, Universitá di Napoli "Federico II", Via Cinthia, 80126 Napoli, Italy

59 Istituto Nazionale di Fisica Nucleare (INFN), sez. di Napoli, Via Cinthia 9, 80126 Napoli, Italy

60 Department of physics, Waseda University, 3-4-1 Okubo, Shinjuku, Tokyo 169-8555, Japan

61 Dpto. de Fiísica Moderna, Universidad de Cantabria, Avda. los Castros s/n, 39005 Santander, Spain

62 Departamento de Física Fundamental and IUFFyM, Universidad de Salamanca, 37008 Salamanca, Spain

${ }^{63}$ Centro de Astrobiología (CSIC-INTA) ESA - ESAC - Villanueva de la Cañada, Madrid, Spain 


\section{Appendix A: Log of miniJPAS observations}

In this appendix, we provide some information on the data aquisition. More details on each tile can be found in the database in the ADQL table minijpas.TileImage. In Table A.1 we indicate the number of exposures taken for each filter in each pointings (red: miniJPAS-AEGIS1, blue: miniJPAS-AEGIS2, green: miniJPAS-AEGIS3, orange: miniJPAS-AEGIS4). Different groups of filters have been observed in different observational blocks (FW\#), following the chosen strategy of filter changes in the JPAS-PF filter wheel (see Sect. 2.4). Total exposure times per pointing are reported in the last column. 


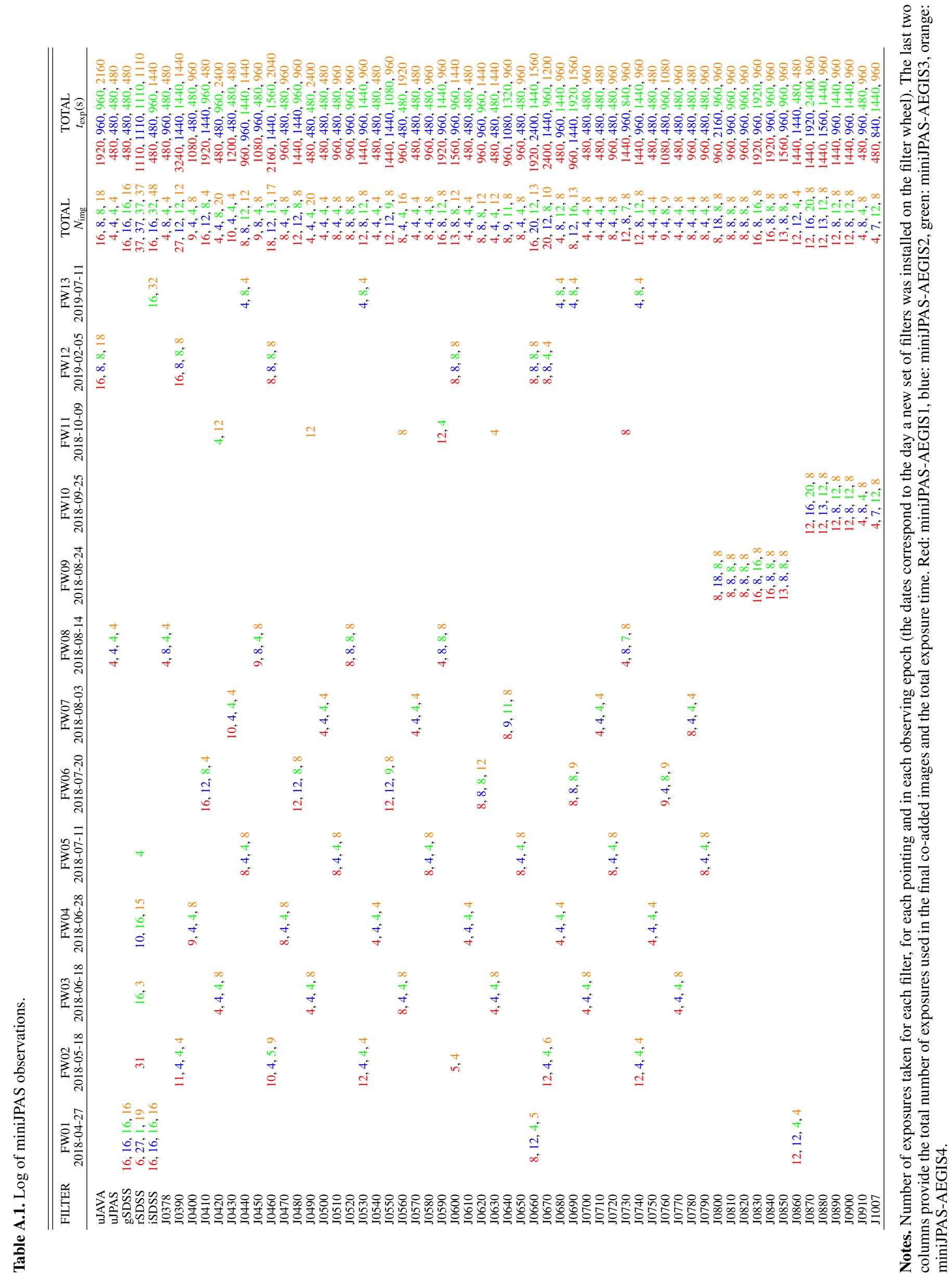




\section{Appendix B: Background correction}

Here, we provide more technical details on the background treatment of the miniJPAS images discussed in Sect. 3.1. In particular, we describe a background correction based on the illumination correction as well as a "superbackground" correction.

\section{B.1. Background correction derived from illumination correction}

The illumination correction is becoming a standard step in the image reduction chain when the acquisition system involves large FoVs or includes lenses like field correctors. Such telescopes are prone to illumination issues (Andersen et al. 1995). Nowadays, projects involving that type of telescopes take that effect into account (e.g., DES, Bernstein et al. 2017 and SDSS, Betoule et al. 2013). The approach adopted for the illumination correction in the OAJ reduction pipeline is based on Manfroid (1995), requiring the acquisition of a set of specific observations. A master illumination correction image (ICOR, hereafter) was constructed for each filter and applied to the images just after the flat field correction ${ }^{42}$. However, images corrected by the ICOR showed a background pattern not visible after the preceding flat field correction. Before continuing with the description of the procedure for removing this apparent degradation of the images after the illumination correction, we will explain its origin.

Due to the optics of the system, a flat illumination arriving to the telescope can result in a non uniform distribution of light on the detector. Scientific images can be affected in two ways. First, they show a non uniform background pattern which is an additive component that should be subtracted. Second, it affects the flat field correction. If it is not taken into account, the nonuniform distribution of light in the flat field images is assumed to be a variation of the efficiency (which it is not). This has two effects on the scientific images after being divided by the master flat. First, it introduces a 2D variation of the photometry of the objects (i.e., a $2 \mathrm{D}$ variation of the photometric zero point). This is the effect corrected by the ICOR. The second effect is to apparently remove the previous background pattern of the scientific images. However, this background should be corrected by subtraction and not by division and, therefore, this apparent background removal is not correct. And, in fact, any additive background present in the raw image, should still be present in the images after flat field correction. That is the reason why after the illumination correction, which corrects the flat field itself, the original additive background pattern present in the raw image reappears in the scientific image.

Knowing that there is a background component of the images which has the same spatial distribution as the ICOR and that it is an additive component, a new background correction is computed having the 2D distribution of the ICOR and an average value of the average background of the target image. This background correction is subtracted just after applying ICOR resulting in a reduced image with flatter background and flatter zero point.

Finally, to check that this background subtraction did not affect the actual photometry of the objects of the images, we processed the individual images used for constructing an ICOR, checking that the differences in the photometry of individual objects observed in different images were compatible with zero.

42 The procedure is equivalent to the correction of the actual flat field.

\section{B.2. Superbackground correction}

After the background subtraction from the ICOR, many single frames still display background patterns whose spatial distribution made them difficult to be properly managed in a massive automatic way during the source detection process with SExtractor. These patterns have two main characteristics that make the correction complex: presence of strong background gradients and strong temporal variability of the patterns, either in intensity or spatial distribution ${ }^{43}$.

Under the assumptions that these patterns are instrumental and, therefore, do not depend on the sky position (at least for close enough pointings), and that they are stable on time scales of a few minutes, we can consider that images taken close in time (with the same filter) present the same background pattern. Therefore, a median combination of scientific images with celestial objects in different CCD positions should keep the background pattern while removing the real astrophysical objects. This is a procedure analogous to the construction of master fringing images or superflat images, and for this reason we dubbed these background images "superbackground images" (SBKG, hereafter).

The observational strategy of miniJPAS where the single exposures of the same pointing and same filter were taken with small dithers one after the other required us to avoid contaminating real sources falling on the parts of the images used to construct the SBKG. Since we could not combine images with the same filter from different pointings, due to temporal variation of the patterns, we had to use the images of the same pointing. Given the small dithering pattern this increased the chances of having areas of the image covered by actual objects, especially by larger diffuse objects. To minimize this possibility, before combining the images, the objects detected in a preliminary reduction without SBKG correction were masked with masks a bit larger than the actual detected size of the object to take into account extended diffuse light. This masking procedure was fine-tuned until it was checked that there were no residuals of extended galaxies in the SBGK (for bright saturated stars we allowed for light of their halos to be in the SBKG). In the specific case of miniJPAS, a field devoid of large galaxies (i.e., galaxies occupying a large area of the CCD), the possible oversubtraction of background due to this procedure was not a big issue, although for studies of faint extended sources this has to be kept in mind.

In summary, the SBKG construction consisted, first, of identifying the images to be combined. That was done by taking advantage that exposures of the same pointing and same filter were done consecutively and, therefore, we could consider that in the interval between the first and last exposure the background pattern was stable (although in a few occasions we detected that this was not the case and some residuals were left). Then, objects were masked and images median-combined. Finally, the resulting image was smoothed using SExtractor. The last step was to subtract the SBKG from the individual scientific frames.

Again, in order to verify that the SBKG correction did not affect the photometry of the objects, we simulated the construction of an ICOR (a procedure that by construction shows changes in photometry of objects across the CCD) applying all the corrections to the images to be used, finding that the resulting map was compatible with no correction.

\footnotetext{
43 It is due to this temporal variation that they are not removed by the background coming from the ICOR which only removes the stable illumination common to flat field and scientific images.
} 


\section{Appendix C: SExtractor's parameters}

Source detection and basic measurements of the objects in the images was done using SExtractor Bertin \& Arnouts (1996). Details on how SExtractor works can be found in Bertin \& Arnouts (1996) and in the SExtractor User Manual. In Table C.1, we provide the list of parameters that were used to obtain the photometric catalogues that are the base of this data release. We only list those parameters that are common to all the images. Other parameters, such as CATALOG_NAME and CHECKIMAGE_NAME, are specific to each image and are not included in the table.
Table C.1. SExtractor's parameters used to construct the miniJPAS source catalogues.

\begin{tabular}{lr}
\hline \hline SExtractor parameter & Value \\
\hline ANALYSIS_THRESH & 2.0 \\
BACKPHOTO_THICK & 24 \\
BACKPHOTO_TYPE & LOCAL \\
BACK_FILTERSIZE & 3 \\
BACK_FILTTHRESH & 0.0 \\
BACK_SIZE & 512 \\
BACK_TYPE & AUTO \\
BACK_VALUE & $0.0,0.0$ \\
CLEAN & $\mathrm{Y}$ \\
CLEAN_PARAM & 1.0 \\
DEBLEND_MINCONT & 0.005 \\
DEBLEND_NTHRESH & 32 \\
DETECT_MINAREA & 5 \\
DETECT_THRESH & 0.9 (dual-mode) / 2 (single-mode) \\
DETECT_TYPE & CCD \\
FILTER & $\mathrm{Y}$ \\
FILTER_NAME & gauss_3.0_5x5.conv \\
GAIN_KEY & GAIN \\
MASK_TYPE & CORRECT \\
PHOT_AUTOAPERS & $0.0,0.0$ \\
PHOT_AUTOPARAMS & $2.5,3.5$ \\
PHOT_FLUXFRAC & 0.5 \\
PHOT_PETROPARAMS & $2.0,3.5$ \\
PIXEL_SCALE & 0.2267 \\
STARNNW_NAME & default.nnw \\
THRESH_TYPE & RELATIVE \\
VERBOSE_TYPE & NORMAL \\
WEIGHT_GAIN & Y \\
WEIGHT_THRESH & 0,0 \\
WEIGHT_TYPE & MAP_WEIGHT,MAP_WEIGHT \\
\hline &
\end{tabular}

